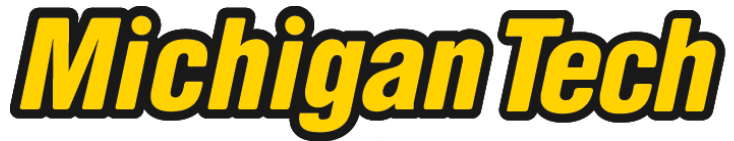 \\ Michigan Technological University Create the Future Digital Commons @ Michigan Tech
}

Dissertations, Master's Theses and Master's Reports - Open

Dissertations, Master's Theses and Master's

Reports

2010

\section{Quantification of relativistic perturbation forces on spacecraft trajectories}

Nicholas Palma Mastricola

Michigan Technological University

Follow this and additional works at: https://digitalcommons.mtu.edu/etds

Part of the Mechanical Engineering Commons

Copyright 2010 Nicholas Palma Mastricola

\section{Recommended Citation}

Mastricola, Nicholas Palma, "Quantification of relativistic perturbation forces on spacecraft trajectories", Master's Thesis, Michigan Technological University, 2010.

https://doi.org/10.37099/mtu.dc.etds/386

Follow this and additional works at: https://digitalcommons.mtu.edu/etds

Part of the Mechanical Engineering Commons 


\title{
QuANTIFICATION OF ReLATIVISTIC \\ Perturbation Forces on Spacecraft Trajectories
}

\author{
By \\ Mr. Nicholas Palma Mastricola
}

\author{
A Thesis \\ Submitted in partial fulfillment of the requirements \\ for the degree of \\ Master of Science in Engineering Mechanics
}

Michigan Technological University

2010

(C) MMX N. P. Mastricola 

This thesis, "Quantification of Relativistic Perturbation Forces on Spacecraft Trajectories," is hereby approved in partial fulfillment of the requirements for the degree of Master of Science in Engineering Mechanics.

Department of Mechanical Engineering - Engineering Mechanics

Signatures:

Thesis Advisor:

Dr. Ossama Abdelkhalik

Department Chair:

Dr. William W. Predebon

Date: 

I would like to dedicate this work to my parents, Rose Marie and John Mastricola, for their continuous support and encouragement throughout my educational career. 



\section{Contents}

List of Figures $\quad$ xi

List of Tables $\quad$ XV

Acknowledgments $\quad$ xvii

Abstract $\quad$ xix

1 Introduction and Background 1

1.1 Motivation . . . . . . . . . . . . . . . . . 1

1.2 Gravitational Theories in General . . . . . . . . . . . . . . 3

1.3 Non-Relativistic Theory . . . . . . . . . . . . . . . . . 4

1.3.1 Keplerian Mechanics ................ 4

1.3.2 Newtonian Mechanics . . . . . . . . . . . . . . 6

1.4 Special Relativity . . . . . . . . . . . . . . . . . . . 7

1.4.1 Galilean Relativity ... . . . . . . . . . . . 7

1.4.2 The Lorentz Transformation . . . . . . . . . . . . . . . . . 8

1.5 General Relativistic Theory . . . . . . . . . . . . . . 9 9

1.5.1 The Principle of Equivalence . . . . . . . . . . . . . . . . 9

1.5.2 The Principle of General Covariance . . . . . . . . . . . . . . 10

1.5.3 Spacecraft Relativistic Equations of Motion . . . . . . . . . . 14

2 Orbit Propagation and Perturbation $\quad 19$ 
2.1 Orbit Propagation . . . . . . . . . . . . . . . . . . . . . 19

2.1.1 Analytical Propagation of EoMs . . . . . . . . . . . 19

2.1 .2 Numerical Integration of EoMs _ . . . . . . . . . . 20

2.1.3 Modeling Perturbation Accelerations _. . . . . . . . . 21

2.1.3.1 General Perturbation Techniques .......... 22

2.1.3.2 Special Perturbation Techniques . . . . . . . . . 22

2.2 Orbit Perturbation . . . . . . . . . . . . . . . . . 23

2.2 .1 Central Body Terms . . . . . . . . . . . . . . . . 23

2.2.2 Lense-Thirring and Rotational Energy Terms . . . . . . . 24

2.2 .3 Geodesic Precession Term . . . . . . . . . . . . . 26

3 Numerical Results $\quad 29$

3.1 The Test Case Orbits . . . . . . . . . . . . . . . . . . . . . . . . . . . 29

3.2 Relativistic Oblateness Orbital Element Data . . . . . . . . . 30

3.2 .1 The Standard Case . . . . . . . . . . . . . . . . . . 31

3.2.1.1 Periodic Orbital Elements . . . . . . . . . . . . . 31

3.2.1.2 Secular Orbital Elements _. . . . . . . . . . 32

3.2 .2 Molniya Orbit Case . . . . . . . . . . . . . . . . . . . . 33

3.2.2.1 Periodic Orbit Elements . . . . . . . . . . . . . 33

3.2.2.2 Secular Orbit Elements . . . . . . . . . . . . 34

3.3 Effects of Standard Oblateness on Orbital Elements . . . . . . . . 35

3.3 .1 Standard Orbit . . . . . . . . . . . . . 35

3.3 .2 Molniya Orbit Results . . . . . . . . . . . . . . . 36

3.4 Discussion of Orbital Element Data . . . . . . . . . . . . . 38

3.4 .1 Data Trends Analysis . . . . . . . . . . . . . . . . . . 38

3.4 .2 Discussion of Data Values . . . . . . . . . . . . . . . 39

3.5 Discussion of Specific Force Data . . . . . . . . . . . . . 41

3.5.1 Relativistic Oblateness Perturbation _. . . . . . . . 42 
3.5.1.1 Standard Orbit Case . . . . . . . . . . . . . . 42

3.5.1.2 Molyina Orbit Case . . . . . . . . . . . . . . 42

3.5.2 Standard Oblateness Perturbation . . . . . . . . . . . . . . 43

3.5.2.1 Standard Orbit Case . . . . . . . . . . . . . 43

3.5.2.2 Molyina Orbit Case . . . . . . . . . . . . . 43

3.5.3 Comparison of Specific Force Data _ . . . . . . . . . 46

4 Conclusions $\quad 47$

4.1 Proof of Concept . . . . . . . . . . . . . . . . . . . 47

4.2 Relevance of Presented Work . . . . . . . . . . . . . . . . 48

4.3 Implications and Future Work . . . . . . . . . . . . . . 49

$\begin{array}{ll}\text { Appendices } & 51\end{array}$

A Abbreviations $\quad 53$

$\begin{array}{ll}\text { B Symbols } & 55\end{array}$

$\begin{array}{ll}\text { C Properties of Earth } & 57\end{array}$

$\begin{array}{ll}\text { D Theoretical Propagation Code } & 59\end{array}$

E Unperturbed State Space Code $\quad 63$

$\begin{array}{lll}\text { F } & \text { Modified State Space I } & 65\end{array}$

$\begin{array}{ll}\text { G Modified State Space II } & 67\end{array}$

$\begin{array}{ll}\text { H Commonly Used Equations } & 69\end{array}$

I The Twins Problem Solution $\quad 71$

$\begin{array}{lll}\text { J Relativistic Perturbation Code } & 73\end{array}$ 
$\begin{array}{ll}\text { K Relativistic Perturbation Data for Standard Case } & 79\end{array}$

K.1 Orbital Parameter Data . . . . . . . . . . . . . . . . . . . . . 79

K.2 Specific Force Data . . . . . . . . . . . . . . . . . . . 86

L Relativistic Perturbation Data for Molniya Case $\quad 89$

L.1 Orbital Parameter Data . . . . . . . . . . . . . . . . . . . . . . . . . 89

L.2 Specific Force Data . . . . . . . . . . . . . . . . . 96

$\begin{array}{ll}\text { Bibliography } & 99\end{array}$ 


\section{List of Figures}

1.1 Kepler's Laws' Geometry (Adapted from [32]) . . . . . . . . . . . 5

1.2 Geometry of the Galilean Transformation . . . . . . . . . . . . . 7

1.3 Elevator Thought Experiment . . . . . . . . . . . . . . . 10

1.4 Position Vector Expressed in Two Frames of Reference . . . . . . . . 11

1.5 Planet with Multiple Accelerating Reference Frames . . . . . . . . . . 12

1.6 Twins Paradox Setup . . . . . . . . . . . . . . . . . . . . . . 13

2.1 Analytical Propagator Architecture . . . . . . . . . . . . . . . 20

2.2 Two Dimensional Space-Times . . . . . . . . . . . . . . . . 25

3.1 Standard Orbit Angular Differences for Relativistic Oblateness versus Time ........................ 31

3.2 Standard Orbit Distance Differences for Relativistic Oblateness versus Time . . . . . . . . . . . . . . . . . . . 32

3.3 Molniya Orbit Angular Differences for Relativistic Oblateness versus

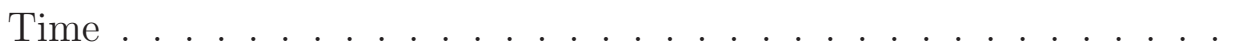

3.4 Molniya Orbit Distance Differences for Relativistic Oblateness versus Time.

3.5 Standard Orbit Angular Differences for Standard Oblateness Pert. versus Time . . . . . . . . . . . . . . . . .

3.6 Standard Orbit Distance Differences for Standard Oblateness Pert. versus Time . . . . . . . . . . . . . . . . . . . . . 36

3.7 Molniya Orbit Angular Differences for Standard Oblateness Pert. versus Time . . . . . . . . . . . . . . . . . . . . 
3.8 Molniya Orbit Distance Differences for Standard Oblateness Pert. versus Time . . . . . . . . . . . . . . . . . . . 37

3.9 Standard Orbit Specific Force for Relativistic Oblateness versus Time 42

3.10 Molniya Orbit Specific Force for Relativistic Oblateness versus Time . 43

3.11 Standard Orbit Specific Force for Standard Oblateness versus Time . 45

3.12 Molniya Orbit Specific Force for Standard Oblateness versus Time . . 45

C.1 Earth Geometry and Coordinate System . . . . . . . . . . 57

K.1 Standard Orbit Angular Differences for Spherical Perturbation versus Time . . . . . . . . . . . . . . . . . . . . . 81

K.2 Standard Orbit Distance Differences for Spherical Perturbation versus Time . . . . . . . . . . . . . . . . . . . . . . 81

K.3 Standard Orbit Angular Differences for Relativistic Oblateness versus Time . . . . . . . . . . . . . . . . . . . . . 82

K.4 Standard Orbit Distance Differences for Relativistic Oblateness versus Time . . . . . . . . . . . . . . . . . . . 82

K.5 Standard Orbit Angular Differences for Lense-Thirring versus Time . 83

K.6 Standard Orbit Distance Differences for Lense-Thirring versus Time . 83

K.7 Standard Orbit Angular Differences for Rotational Energy versus Time 84

K.8 Standard Orbit Distance Differences for Rotational Energy versus Time 84

K.9 Standard Orbit Angular Differences for Geodesic versus Time $\ldots . \quad 85$

K.10 Standard Orbit Distance Differences for Geodesic versus Time . . . 85

K.11 Standard Orbit Specific Force for Spherical Perturbation versus Time 86

K.12 Standard Orbit Specific Force for Relativistic Oblateness versus Time 87

K.13 Standard Orbit Specific Force for Lense-Thirring versus Time $\ldots . \quad 87$

K.14 Standard Orbit Specific Force for Rotational Energy versus Time . . 88

K.15 Standard Orbit Specific Force for Geodesic versus Time . . . . . . 88

L.1 Molniya Orbit Angular Differences for Spherical Perturbation versus Time . . . . . . . . . . . . . . . . . . . . 91 
L.2 Molniya Orbit Distance Differences for Spherical Perturbation versus

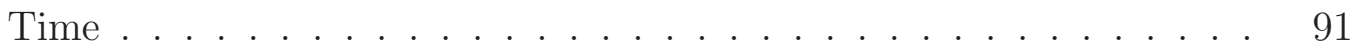

L.3 Molniya Orbit Angular Differences for Relativistic Oblateness versus Time ......................... 92

L.4 Molniya Orbit Distance Differences for Relativistic Oblateness versus Time . . . . . . . . . . . . . . . . . . . 92

L.5 Molniya Orbit Angular Differences for Lense-Thirring versus Time . . 93

L.6 Molniya Orbit Distance Differences for Lense-Thirring versus Time . 93

L.7 Molniya Orbit Angular Differences for Rotational Energy versus Time 94

L.8 Molniya Orbit Distance Differences for Rotational Energy versus Time 94

L.9 Molniya Orbit Angular Differences for Geodesic versus Time . . . . . 95

L.10 Molniya Orbit Distance Differences for Geodesic versus Time . . . . . 95

L.11 Molniya Orbit Specific Force for Spherical Perturbation versus Time . 96

L.12 Molniya Orbit Specific Force for Relativistic Oblateness versus Time . 97

L.13 Molniya Orbit Specific Force for Lense-Thirring versus Time . . . . . 97

L.14 Molniya Orbit Specific Force for Rotational Energy versus Time . . . 98

L.15 Molniya Orbit Specific Force for Geodesic versus Time . . . . . . . . 98 


\section{List of Tables}

3.1 Orbital Parameters for Test Orbit Cases . . . . . . . . . . . 30

3.2 Periods of Test Orbits . . . . . . . . . . . . . . . . 30

3.3 Secular Element Trends for Relativistic Oblateness _ . . . . . . . . 32

3.4 Trends of the Secular Elements for the Molniya Orbit . . . . . . . . 34

3.5 Summary of Data Trends . . . . . . . . . . . . . . . . . . . . 38

3.6 Summary of Data Values . . . . . . . . . . . . . . . . . . . 40

3.7 Summary of Force Data Values in Newtons . . . . . . . . . . . . 46

C.1 Earth Parameters . . . . . . . . . . . . . . . . . . . . . 58

K.1 Summarized Orbital Parameter Data for Standard Orbit . . . . . 79

K.2 COE Statistics for Standard Orbit Case . . . . . . . . . . . 80

K.3 Specific Force Magnitude Data Summary for Standard Case . . . . 86

L.1 Summarized Orbital Parameter Data for Molniya Orbit . . . . . . . 89

L.2 COE Statistics for Molniya Orbit Case . . . . . . . . . . . 90

L.3 Specific Force Magnitude Data Summary for Molniya Orbit Case . . 96 


\section{Acknowledgments}

There are several people without whom this work would not have been possible. Firstly, I would like to thank my advisor, Dr. Ossama Abdelkhalik. He was always available to give direction, guidance, or a kind word of support when necessary.

I would like to thank Prof. Chris E. Passerello and Prof. Clark R. Givens for agreeing to serve on my committee. Prof. Passerello was also kind enough to always make himself available to answer questions about the theoretical aspects of my project. Prof. Givens also made himself available to help me address some of the difficult computational aspects of The General Theory of Relativity.

Dr. Jason Dreyer was also instrumental during the writing process. He was always willing to give advice on the structure of my manuscript and provide tips that helped to streamline the writing process.

I would like to recognize my mother, Dr. Rose Marie Mastricola, for her help with the arduous task of editing this manuscript. My mother, my father, John Mastricola, and my brother, Anthony Mastricola, were always at the ready with a kind word of encouragement or advice on how to deal with difficult situations as they arose throughout the course of this work. I am truly lucky to have had the support of my family in all aspects of this endeavor. I am eternally grateful for everything that they all have done, and would like to say thank you with my deepest sense of gratitude.

Lastly, but certainly not least, I would like to thank my friend, Riley Strehlow. He was monumental in my preparation for the defense of this work. He was also kind enough to help with the editing process of this manuscript - albeit, he may not have known what he was getting himself into. He was always ready to act as a sounding board for ideas, give a kind word of support, or have a few drinks and some good conversation at the bar to decompress after a long day in the office. I am, and always will be, grateful for his support and our friendship. 


\section{Abstract}

The intent of the work presented in this thesis is to show that relativistic perturbations should be considered in the same manner as well known perturbations currently taken into account in planetsatellite systems. It is also the aim of this research to show that relativistic perturbations are comparable to standard perturbations in specific force magnitude and effects. This work would have been regarded as little more then a curiosity to most engineers until recent advancements in space propulsion methods - e.g. the creation of a artificial neutron stars, light sails, and continuous propulsion techniques. These cutting-edge technologies have the potential to thrust the human race into interstellar, and hopefully intergalactic, travel in the not so distant future.

The relativistic perturbations were simulated on two orbit cases: (1) a general orbit and (2) a Molniya type orbit. The simulations were completed using Matlab's ODE45 integration scheme. The methods used to organize, execute, and analyze these simulations are explained in detail. The results of the simulations are presented in graphical and statistical form. The simulation data reveals that the specific forces that arise from the relativistic perturbations do manifest as variations in the classical orbital elements. It is also apparent from the simulated data that the specific forces do exhibit similar magnitudes and effects that materialize from commonly considered perturbations that are used in trajectory design, optimization, and maintenance.

Due to the similarities in behavior of relativistic versus nonrelativistic perturbations, a case is made for the development of a fully relativistic formulation for the trajectory design and trajectory optimization problems. This new framework would afford the possibility of illuminating new more optimal solutions to the aforementioned problems that do not arise in current formulations. This type of reformulation has already showed promise when the previously unknown Space Superhighways arose as a optimal solution when classical astrodynamics was reformulated using geometric mechanics. 


\section{Chapter 1}

\section{Introduction and Background}

The General Theory of Relativity (GTR) has traditionally been looked at and utilized in the realms of theoretical and mathematical physics. In fact, most engineers would not even consider the possibility that relativistic and quantum mechanics may be affecting the system that is under consideration. However, taking recent advancements made in the areas of space propulsion systems and applied physics into account, it is possible for one to ponder the possibility that one day we will have the capability to reach beyond our solar system with interstellar, and hopefully intergalactic, travel.

\subsection{Motivation}

The problems of space trajectory design and optimization have been included in the realm of engineering since the mid 1920s when Walter Hohmann published his work on trajectory design, The Attainability of Heavenly Bodies. Within this work, Hohmann suggests a minimum change in velocity, or minimum energy, solution [32]. Hohmann's work was rooted in classical mechanics (non-relativistic) [17]. It is no surprise that the work based off of Hohmann's original book also utilized this framework even though there have been significant advancements and a multitude of reformulations.

Trajectory optimization has its beginnings firmly rooted in the Calculus of Variations $(\mathrm{CoV})$ [20]. It is therefore possible to define the fundamental problem in orbit optimization as the integral seen in Equation 1.1.

$$
I=\int_{0}^{1} f(\vec{x}) d x
$$

$I$ is known as the functional. It is a function of the state variables and their deriva- 
tives, which are represented by $\vec{x}$. The goal of the CoV optimization approach is to minimize $I$ by finding a set of admissible curves [20]. An example of this type of optimization in two dimensions is the brachistochrone - a classical problem in the $\mathrm{CoV}$ [27]. The true power and breadth of this optimization technique is not fully recognized until multi-parameter highly nonlinear optimization problems are considered $[2]$.

All the work done in orbit optimization is not contained within the analytical realm of the CoV. Several advancements have also been made on numerical optimization techniques [25] as well as geometric techniques [5]. Several of these techniques are just beginning to be applied to orbit optimization. These include, but are not limited to: genetic algorithms, geometric optimal control, direct and global search methods. Each of the methods mentioned here are capable of handling searches over multi-dimensional solution spaces.

The GTR becomes more applicable to the aforementioned engineering problems as new technologies are developed that increase the distance and speed capabilities of long range spacecraft. An example of this advanced technology is Sandia National Laboratories' Z-machine [33]. The Z-machine has been able to create an artificial "star" that behaves in the same manner as a black hole [3]. This result has two important implications: (1) the age of fusion power is drawing nearer, and (2) manipulation of the fabric of space-time is possible through artificial means. The technologies given rise to by either of these implications would demand that the formulation of trajectory design and optimization problems must be examined within the framework of relativistic mechanics. This research is of great importance because of the possibility of the existence of new solutions to the problems of trajectory design and trajectory optimization that do not arise in the classic Newtonian theory and may manifest themselves only in the frameworks of special or general relativity.

The idea of using non-traditional frameworks to see if new and different solutions arise is not a new one. An example of this is the discovery of the interplanetary superhighway by Lo and Ross in 1997 [21]. The method they employed was to utilize halo orbits around Lagrange points as a basis for an invariant manifold [22]. Using geometric techniques, they were able to show which of the manifolds were stable and could be used for optimal orbital maneuvers [22].

Fusion power does have the possibility of providing sufficient acceleration for a macroscopic object to near light speed, but it is regarded as a brute-force technique [24]. The idea of distorting the fabric of space-time on a local level is not a new one. Most people only associate this idea with science fiction. However, this idea is rapidly becoming science fact. Being able to create an artificial neutron star would allow for the warping of space time because of the high gravity [3]. With this in mind, it is possible to talk about applications of this technology. One such technology is the "warp drive." The idea of this technology is that the local geometry of space- 
time could be sufficiently warped to allow for an observer to travel an arbitrarily large distance in an arbitrarily short and finite time [1]. One could think that the observer is traveling at superluminal (or tachyonic) speeds. This is not true because the observer does not leave their initial light cone; therefore, the observer is still traveling below light speed in the local sense [1]. Locally distorting the fabric of space-time also implies that it may be possible to create an artificial wormhole by which spacecraft could travel through interconnected regions of space at superluminal speeds [24].

With the age of interstellar travel drawing closer, it is necessary for engineers to understand the differences between relativistic and Newtonian astrodynamics in preparation for the application of these technologies. Even though these technologies have several decades of development to go, engineers must have the same forethought that Walter Hohmann did when he published his aforementioned text more than thirty years before the launch of Earth's first artificial satellite - Sputnik.

\subsection{Gravitational Theories in General}

There are two fundamental gravitational theories. They are quantum gravitational theory and classical, or non-quantum, gravitational theories. Classical gravitational theories will be sufficient to develop the ideas presented in this thesis. Classical gravitational theories can be dichotomized into two frameworks: (1) Newtonian mechanics and (2) relativistic mechanics. Within the framework of Newtonian mechanics arises what is commonly referred to Galilean relativity. Relativistic mechanics includes the formulations for special relativity, general relativity, and Post-Newtonian ideas. These formulations have lead to models that include several gravitomagnetic effects that do not arise in the Newtonian theory.

It is a common misconception among the general populous that Newtonian mechanics was proven incorrect by Einstein's GTR; however, this is incorrect. Newtonian theory can be thought of as a low energy and low velocity approximation to the more general framework of the GTR [26]. In more succinct terms, the GTR must reduce to the Newtonian theory under the correct limit. Two fundamental principles of mechanics must be applied in order to show that the GTR does reduce to the Newtonian theory under the appropriate limit. They are: (1) the principle of equivalence, and (2) the principle of general covariance 


\subsection{Non-Relativistic Theory}

This section discusses the necessary background information in the non-relativistic theory that will be referenced throughout the remainder of the text.

\subsubsection{Keplerian Mechanics}

Johannes Kepler made several contributions to orbital mechanics throughout his lifetime. His most enduring contributions are his three laws of planetary motion. The following technical explanations of Kepler's have been included for a matter of completeness because they are the basis by which all two-body motion is governed [32].

Kepler's First Law Kepler's first law is stated as "The orbit of each planet is an ellipse with the Sun at one focus" [32]. This law was determined through observational data. However, Kepler now had the issue of relating the planet's position with the orbit's geometry and time. The geometry that was used to derive this and the following laws is seen in Figure 1.1. In Figure 1.1, $\mathbb{O}$ is the origin, $P$ is the point of periapsis, $\mathbb{S} \mathbb{C}$ is the spacecraft's position on the actual orbit, and $\mathbb{S} \mathbb{C}^{\prime}$ is the spacecrafts fictitious position on the auxiliary circle. The rest of the symbols in Figure 1.1 will be explained as they appear in the following equations. A table of symbols has been included in Appendix B has been included for the readers' convenience.

Kepler's Second Law Kepler's second law is stated as "The line joining the planet to the Sun sweeps out equal areas in equal times" [32]. Kepler again used his observational data to determine this. Mathematically speaking, this law can be expressed by Equation 1.2.

$$
\frac{t-T}{A_{1}}=\frac{\mathcal{P}}{\pi a b},
$$

where $t$ is time, $T$ is the time at periapsis, $A_{1}$ (as seen in Figure 1.1) is the area swept out by the orbiting body in the prescribed time, $\mathcal{P}$ is the orbital period, $a$ is the semi-major axis of the orbit, and $b$ is the semi-minor axis of the orbit.

Kepler was able to derive the following relationship using this law and geometric properties of ellipses. Equation 1.3 is known as Kepler's Equation.

$$
M=E-e \sin (E)=\sqrt{\frac{\mu}{a^{3}}}(t-T),
$$

In Equation 1.3, $M$ is the mean anomaly and $E$ is the eccentric anomaly. The eccentric anomaly is found via the auxiliary circle seen in Figure 1.1. The mean anomaly is also an angle that is measured on the auxiliary circle; however, it is based on $t=T=0$ 


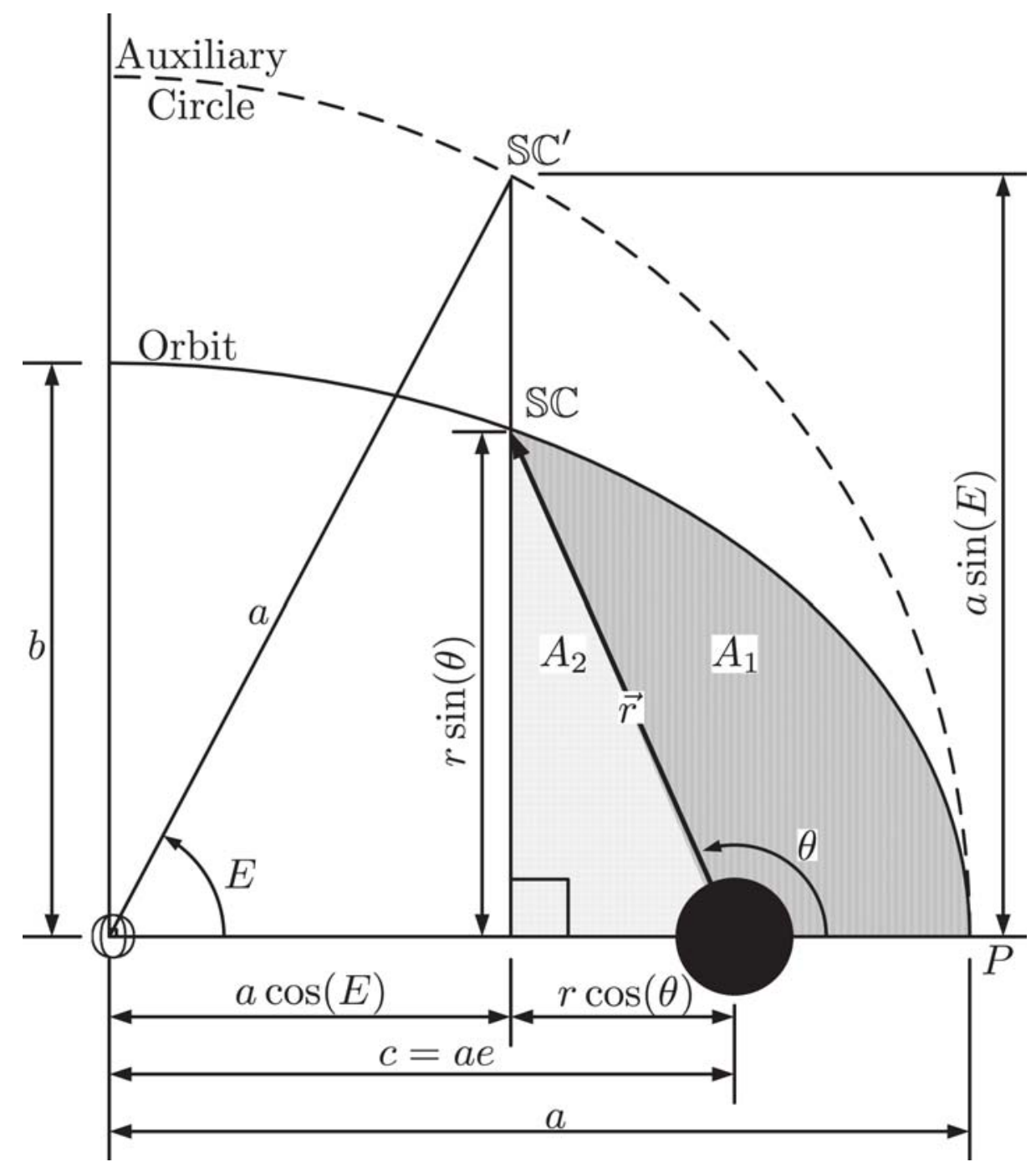

Figure 1.1: Kepler's Laws' Geometry (Adapted from [32])

at the point of pariapsis [32]. The mean anomaly allows time, the eccentric anomaly, and the true anomaly to be related. The eccentric anomaly and true anomaly are related by Equation 1.4.

$$
\sin E=\frac{r \sin \theta}{a \sqrt{1-e^{2}}} \quad \cos E=\frac{a e-r \cos \theta}{a}
$$

The true anomaly, $\theta$, is the measure of the angular distance from the point of pariapsis to the spacecraft's location on the actual orbit (noted as $\mathbb{S C}$ ) on Figure 1.1. 
Kepler's Third Law Kepler's third law is stated as "The square of the period of a planet is proportional to the cube of its mean distance to the Sun" [32]. This law relates the semi-major axis of the orbit to the period. This allows the mean motion of the orbit to be written as a function of $a$. Thus (where $n$ is mean motion),

$$
n=\sqrt{\frac{\mu}{a^{3}}} .
$$

Using this definition for mean motion, it is possible to write the integral expression for the mean anomaly as

$$
M=\int_{T}^{t} n d t=n(t-T) .
$$

\subsubsection{Newtonian Mechanics}

Isaac Newton made several contributions to the various fields of mathematics and physics. One such contribution is what is known as Newton's law of gravitation seen in its full form in Equation 1.7.

$$
\vec{F}_{\mathrm{g}}=-\frac{G m_{\oplus} m_{\mathrm{sat}}}{r^{2}} \hat{r}
$$

This equation is used as the base model for all two-body orbital mechanics problems (i.e. satellites orbiting a central body). In order to manipulate Equation 1.7 into a form that is conducive to modeling satellite orbits, there are four assumptions that must be made and defined. They are [32]:

1. The mass of the satellite is negligible to that of the central body.

2. The coordinates chosen must be an inertial frame.

3. Both bodies in question may be modeled as a point mass.

4. Only gravitational forces are in play.

It is possible to write the kinetic EoM of the satellite using assumption 4 [32]. This is shown in Equation 1.8a. Applying assumption 2 the form seen in Equation $1.8 \mathrm{~b}$ is achieved. The form that is needed is arrived at with one final substitution $\left(\mu_{\oplus}=G m_{\oplus}\right)$.

$$
\begin{aligned}
\vec{F}_{G_{\text {sat }}}=m_{\text {sat }} \ddot{\vec{r}} & =-\frac{G m_{\oplus} m_{\text {sat }}}{r^{2}} \hat{r} \\
\ddot{\vec{r}} & =-\frac{G m_{\oplus}}{r^{2}} \hat{r} \\
\ddot{\vec{r}} & =-\frac{\mu_{\oplus}}{r^{2}} \hat{r}
\end{aligned}
$$


Equation $1.8 \mathrm{c}$ is known as the orbit equation and will be used to develop a state space model for numerical integration (see Section 2.1.2) [32].

\subsection{Special Relativity}

\subsubsection{Galilean Relativity}

The system that Galilean relativity is applicable with consists of two reference frames. The unprimed frame (denoted by $\mathbb{S}$ ) is considered to be a true inertial frame, while the primed frame $\left(\mathbb{S}^{\prime}\right)$ is moving at a constant velocity much smaller than the speed of light. The complete setup may be seen in Figure 1.2 [15].

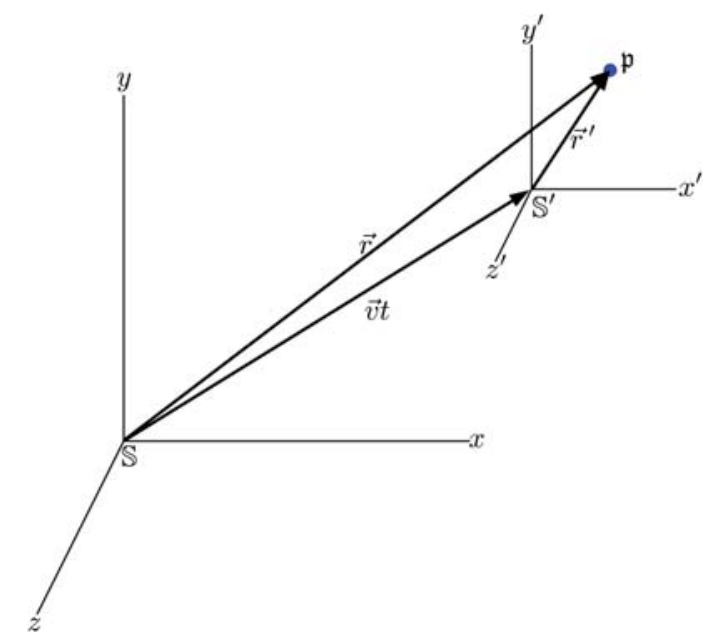

Figure 1.2: Geometry of the Galilean Transformation

The vector equations that arise from the geometry seen in Figure 1.2 are seen in Equation Group 1.9 [15].

$$
\begin{gathered}
\vec{r}^{\prime}=\vec{r}-\vec{v} t \\
\dot{\vec{r}}^{\prime}=\dot{\vec{r}}-\vec{v} \\
\ddot{\vec{r}}^{\prime}=\ddot{\vec{r}}
\end{gathered}
$$

Equation 1.9a is the expression for the position of particle $\mathfrak{p}$. Two subsequent differentiations results in the velocity expression and acceleration expression of particle $\mathfrak{p}$, Equations $1.9 \mathrm{~b}$ and $1.9 \mathrm{c}$ respectively [15]. Equation 1.9c implies that the accelerations expressed in either frame should be equal. Therefore, the Galilean transformation predicts that the velocity of light should change with respect to choice of 
coordinates [15]. This result also came under scrutiny because of Einstein's dictum that the fundamental laws of physics should appear the same to all freely moving observers [15].

This prediction was proven unequivocally false by the famous series of experiments carried out by Michelson and Morley. These experiments showed that the velocity of light remains constant in all directions with respect to any freely moving observer [15]. Therefore, it was necessary to find a set of transformations that would preserve the velocity of light in any possible reference frame [16].

\subsubsection{The Lorentz Transformation}

As mentioned in the previous section, a transformation must be found that preserves the velocity of light in any reference frame. This idea is mathematically expressed in Equation 1.10.

$$
x^{2}+y^{2}+z^{2}-(c t)^{2}=\left(x^{\prime}\right)^{2}+\left(y^{\prime}\right)^{2}+\left(z^{\prime}\right)^{2}-\left(c t^{\prime}\right)^{2}
$$

The transformation found to do this is known as the Lorentz transformation [16]. However, when it was originally derived it was not without its demons. It was known that Newton's equations of motion were only invariant under the Galilean transformation, which (at this point) were being regarded as incorrect [15]. This implies the possibility that Newton's equations of motion, as well as other fundamental laws of physics, may not be preserved under the correct Lorentz transformation [15].

These concerns were put to rest via the equivalence principle (see Section 1.5.1). When the equivalence principle is applied to this situation, it states that the Lorentz transformation must reduce to the Galilean transformation under the Newtonian limit [15]. Therefore, the Galilean transformation should be regarded as a low velocity (relative velocities much less than the speed of light) of the Lorentz transformation.

The Lorentz transformation has multiple, and widely varying, derivations. How-

ever, the result is always the same. The equations for the coordinate relationships of the Lorentz transformation may be seen in Equation Group 1.11.

$$
\begin{gathered}
x^{\prime}=\gamma\left(x+v_{x} t\right) \\
y^{\prime}=y \\
z^{\prime}=z \\
t^{\prime}=\gamma\left(\frac{v}{c^{2}} x+t\right)
\end{gathered}
$$

where $\gamma$ is know as the Lorentz factor and $\beta$ is known as the speed ratio. These terms 
are sometimes referred to as relativistic gamma and relativistic beta. The expressions for these terms are seen in Equation Group 1.12.

$$
\begin{gathered}
\beta=\frac{v}{c} \\
\gamma=\frac{1}{\sqrt{1-\beta^{2}}}
\end{gathered}
$$

\subsection{General Relativistic Theory}

There are several different viewpoints, methods, theories, and techniques within relativistic mechanics. This section provides a concise overview of the background theory, notation, and terms that were used in the work being presented.

\subsubsection{The Principle of Equivalence}

From the work of Galileo, it is a well known and accepted fact in mechanics that gravitational acceleration is independent of an object's mass [16]. However, this statement, and its acceptance in mechanics, is based on the idea that the measurements and theoretical analysis used to illustrate this concept were done with the notion of an inertial frame. The fundamental idea in the GTR is that all frames are relative frames with respect to any individual frame. Therefore, the idea of a "true" inertial frame is abandoned.

Einstein was able to reconcile this through the use of his famous elevator Gedankenexperimente, or thought experiment. The setup for this thought experiment may be seen in Figure 1.3. The elevators, passengers, and the masses contained in each elevator are identical. Each passenger has no way to receive or transmit information to the outside. Therefore, in this instance, each passenger, not knowing anything to the contrary, would regard their elevator as an inertial frame. The only difference between the two elevators is that the elevator in Figure 1.3b is being accelerated upward with a constant acceleration $a$ for an arbitrarily long time. If each passenger would drop their respective masses, and took time and distance measurements, they would be able to calculate the downward acceleration of the mass. Each passenger would agree on the time it took their respective masses to reach the bottom of the elevator; therefore, they would also agree on the calculated downward acceleration of the mass, $g$ [10]. 


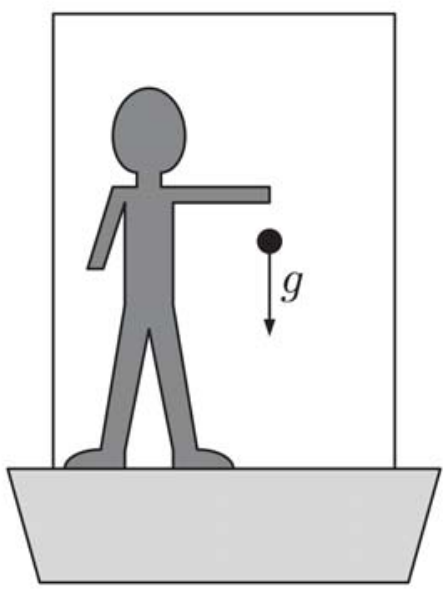

(a) Grounded Elevator

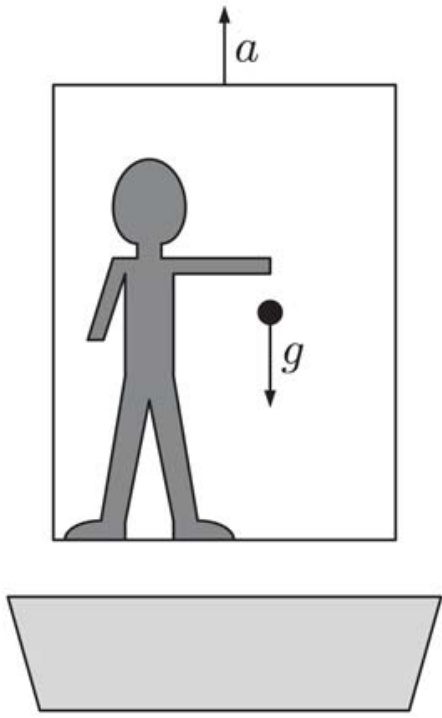

(b) Accelerated Elevator

Figure 1.3: Elevator Thought Experiment

From the results of this thought experiment, it is possible to conclude that a gravitational field acts the same on objects contained within a non-accelerated or an accelerated frame of reference. This is the principle of equivalence in its simplest form.

\subsubsection{The Principle of General Covariance and Use of Ten- sors}

The principle of general covariance is the idea that the fundamental laws of nature must be valid in any coordinate system [10]. In other words, the fundamental laws must be expressible in tensorial form. This is a necessity when working with the GTR because the geometry of space-time is four dimensional and curved; therefore, the geometry is difficult to visualize. The principle of general covariance guarantees that if an equation can be expressed tensorially it will be form-invariant with respect to choice of coordinates [10].

A background quantity that is an invariant must exist, and this quantity must stay invariant under coordinate transformations of the correct type in order for equations to be expressed in tensorial form [30]. An explicative example of this concept in Euclidean two-space is illustrated in Figure 1.4. 


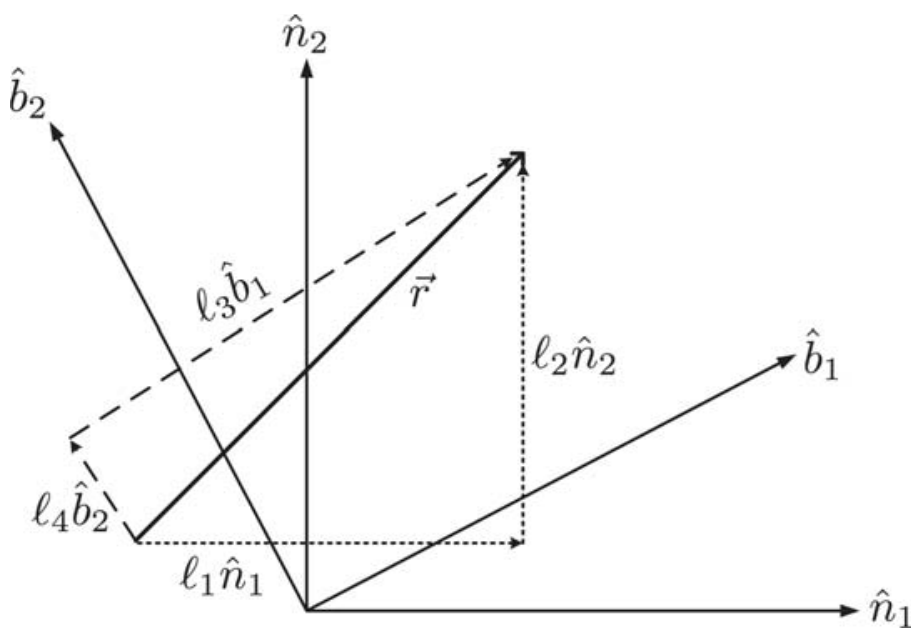

Figure 1.4: Position Vector Expressed in Two Frames of Reference

In Figure 1.4, the dotted vectors represent the components of vector $\vec{r}$ in the $\hat{n}^{-}$ frame and the dashed vectors represent the components in the $\hat{b}$-frame. Since both bases are orthogonal and $\vec{r}$ is embedded in Euclidean two-space, the Pythagorean theorem must hold true [19]. Thus, the dot products may be performed with $\vec{r}$ expressed in either frame. The equivalent dot products are shown in Equation 1.13.

$$
|\vec{r}|=r=\sqrt{{ }_{n} \vec{r} \cdot{ }_{n} \vec{r}}=\sqrt{{ }_{b} \vec{r} \cdot{ }_{b} \vec{r}}
$$

It has now been shown that the invariant of $\vec{r}$ is indeed its magnitude. Now, it would seem that this is a rather inconsequential result. However, this is certainly not the case because one would have to find coordinates that could represent all possible accelerating reference frames [23]. A simplified version of this problem is shown in Figure 1.5.

Each elevator and passenger system in Figure 1.5 is identical and being accelerated by the arbitrary planet's gravitational field. It now can be seen clearly that it is impossible to pick a solitary accelerating reference frame to represent a gravitational field due to the curvature of the field [23]. This is an obstruction to the principle of equivalence explained in Section 1.5.1. The principle of equivalence does hold true locally in what are called Lorentz frames [23].

The principle of general covariance becomes more important because of the aforementioned obstruction. In order to operate in any possible Lorentz frame, relationships between all of the frames must be determined. This is done with Riemannian* geometry when working with the GTR. This requires the introduction to a mathemat-

\footnotetext{
*This is also known as metric, tensor, or differential geometry.
} 


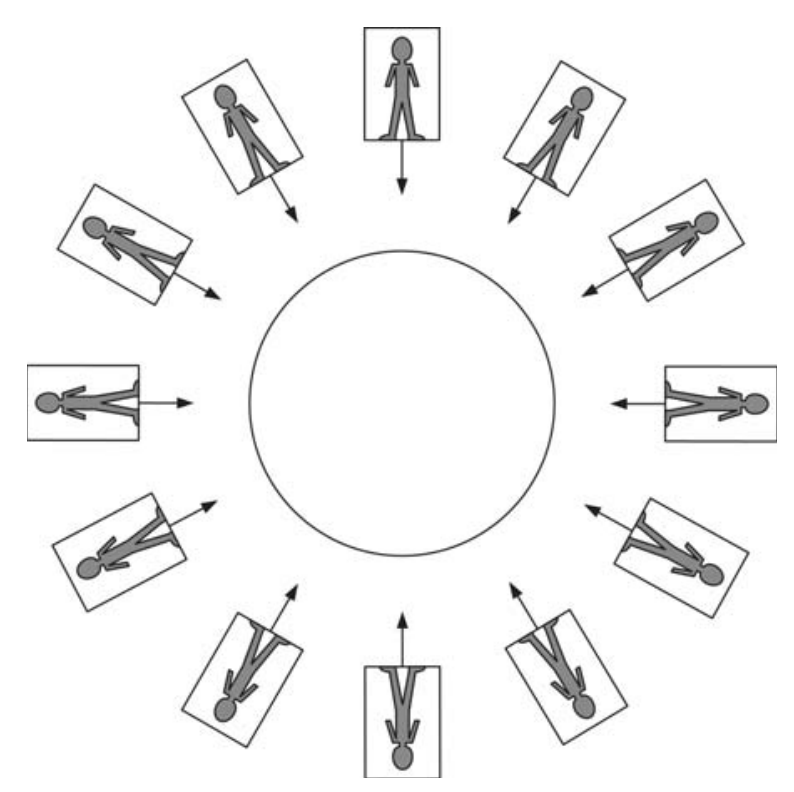

Figure 1.5: Planet with Multiple Accelerating Reference Frames

ical entity known as a metric tensor. The metric tensor allows for the generalization of Pythagorean theorem [30], and may be thought of as a calibrated measuring stick within a space, or on a manifold, that is of interest. Cartesian coordinates will be looked at in order to facilitate this explanation.

In terms of tensor calculus, position vectors in three-space are represented as seen in Equation 1.14.

$$
x^{i}=\left(x^{1}, x^{2}, x^{3}\right) \quad-\text { or }-\quad u^{i}=\left(u^{1}, u^{2}, u^{3}\right)
$$

In Cartesian space, the metric takes the form of the identity tensor $\delta_{i j}{ }^{\dagger}$. This allows Equation 1.13 to be written in indicial notation, with the use of the Einstein summing convention, as seen in Equation 1.15.

$$
r=\sqrt{\delta_{i j} x^{i} x^{j}}=\sqrt{\delta^{i j} x_{i} x_{j}}
$$

Please note that in most engineering texts Equation 1.15 is written in the second form shown because in Euclidean spaces there is no difference between covariant (lower) indices and contravariant (upper) indices. However, this is not the case in non-Euclidean spaces (e.g. four dimensional space-time). The invariant, or preserved, quantity for Euclidean metrics is "something" squared PLUS "something" squared. In four dimensional space-time, the metric must preserve quantities that are "something" squared MINUS "something" squared [10].

\footnotetext{
${ }^{\dagger}$ All Latin indices will run from one to three. Greek indices will run from zero to three.
} 


$$
x^{\mu}=\left\{\begin{array}{l}
x^{0} \\
x^{1} \\
x^{2} \\
x^{3}
\end{array}\right\}=\left\{\begin{array}{l}
t \\
x \\
y \\
z
\end{array}\right\}
$$

The position of an object in four dimensional space-time is represented by the four-vector seen in Equation 1.16. The specific quantity that needs to be preserved is proper time $\tau$ (in relativistic units ${ }^{\ddagger}$ ) seen in Equation 1.17,

$$
\tau^{2}=t^{2}-r^{2}
$$

where $r$ is the magnitude of the three-position vector and $t$ is coordinate time. Proper time is the time that an ideal clock keeps when traveling on a four dimensional path in space-time [10]. Proper time can be thought of as an analog to arc length in three dimensional space. The twins problem can be considered to facilitate explanation. The diagram for the twins problem can be seen in Figure 1.6.

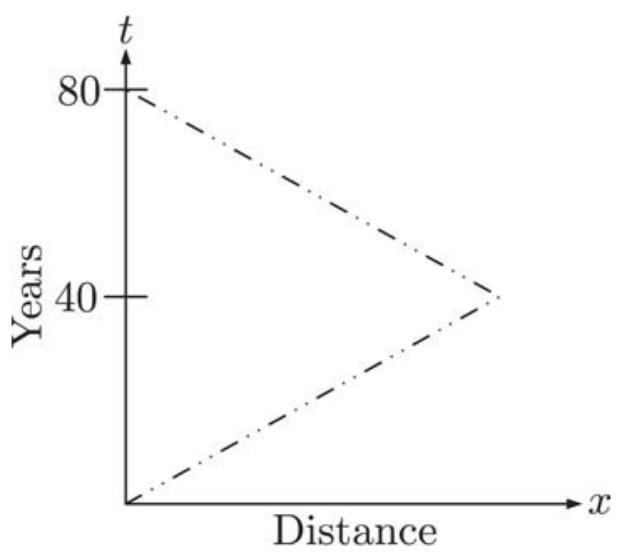

Figure 1.6: Twins Paradox Setup

The setup for the twins problem is in two dimensional space-time (one time coordinate and one spacial coordinate). In this problem, two twins are born at the same time and place. One twin is placed on a spacecraft that travels in a straight line from the twins' place of birth at near superluminal speeds. Meanwhile, the twin who is not on the spacecraft stays at the place of birth. Forty years into the journey, the spacecraft does a full reversal and travels back on the same line to the twins' place of birth at the same speed [10].

The clocks at the twins' place of birth keep coordinate time and travels directly on the $t$-axis. The twin in the spacecraft travels on the dot-dash-dot line in Figure 1.6.

\footnotetext{
$\ddagger$ The units are chosen such that the speed of light, $c$, is equal to one.
} 
The clocks in the spacecraft tick proper time. Therefore, there is a large difference in the amount of time that each twin experiences passing. The twin left at the birth place sees time pass as experienced here on Earth. However, the twin in the spacecraft sees time pass much slower [16]. If the spacecraft is traveling $0.8 c$, the proper time experienced by the traveling twin is forty-eight years (the calculations may be seen in Appendix I). This effect is known as time dilation [16]. Time dilation was experimentally verified by flying an atomic clock around the earth and leaving an identical atomic clock on Earth. Once the clocks were together after the flight, the moving clock was running slower by exactly the amount that was calculated using time dilation [10].

Unfortunately, there is no picture that can be drawn to demonstrate this in spacetime. Therefore, it must be demonstrated analytically. This can be easily done with the Minkowskian metric $\eta_{\mu \nu}$. The Minkowskian metric is the metric of flat space-time [10]. It, in conjunction with Lorentz frames, is used extensively in special relativity [23]. The Minkowskian metric takes the component form seen in Equation 1.18 ${ }^{\S}$

$$
\eta_{\mu \nu}=\left[\begin{array}{cccc}
1 & 0 & 0 & 0 \\
0 & -1 & 0 & 0 \\
0 & 0 & -1 & 0 \\
0 & 0 & 0 & -1
\end{array}\right]
$$

Thus, proper time can be expressed in tensorial form as seen in Equation 1.19.

$$
\tau^{2}=\eta_{\mu \nu} x^{\mu} x^{\nu}
$$

\subsubsection{Spacecraft Relativistic Equations of Motion}

Non-relativistic equations of motion are based on fundamental principles by which physical systems, as well as forces and perturbations acting on the system, may be modeled. Forces and physical properties of the system's components give rise to the motion, and character of motion, of the system. In relativistic theories, much more attention is given to an object's surroundings than in the Newtonian theory [26].

In the GTR there are two entities that need to be addressed. They are the matter embedded within a field, and the field itself [12]. These two nebulous entities manifest themselves in ways that are not distinctly defined [13]. The field (gravitational field to be precise) may be thought of as a background canvas which may be warped and twisted by energy.

Now the question becomes, "How is it possible to deal with a dynamic field and the matter embedded within it?" This again leads to the introduction of the metric,

\footnotetext{
§Please note that the metric is in relativistic units - units system where $c=1$.
} 
as pointed out in Section 1.5.2. In Section 1.5.2, it was noted that Equation 1.18 is the metric tensor components of flat space-time, or the space-time of special relativity. Now the curvature of space-time must be considered because gravity is regarded as a consequence of this curvature [23]. The most basic GTR metric is the metric of a non-rotating charge-free black hole - the Schwarzschild metric. This metric may be seen in Equation 1.20, where $m$ is the Schwarzschild radius [10].

$$
g_{\mu \nu}=\left[\begin{array}{cccc}
\left(1-2 \frac{m}{r}\right) & 0 & 0 & 0 \\
0 & -\left(1-2 \frac{m}{r}\right)^{-1} & 0 & 0 \\
0 & 0 & -r^{2} & 0 \\
0 & 0 & 0 & -r^{2} \sin ^{2}(\theta)
\end{array}\right] \quad \text { where } m=\frac{G M \bullet}{c^{2}}
$$

In the expression for the Schwarzschild radius, $G$ is Newton's constant and $M_{\bullet}$ is the mass of an arbitrary planet.

The line element in this space-time can be calculated by two simple contractions of the metric tensor with the differential length element. Thus,

$$
d s^{2}=g_{\mu \nu} d x^{\mu} d x^{\nu},
$$

where the differential length element $d x^{\mu}$ is represented by the space-time 4 -vector

$$
d x^{\mu}=\left\{\begin{array}{l}
d t \\
d r \\
d \theta \\
d \phi
\end{array}\right\}
$$

The result of these contractions is Equation 1.22, and is known as the Schwarzchild solution [10].

$$
d s^{2}=\left(1-2 \frac{m}{r}\right) d t^{2}+\frac{1}{c^{2}}\left(-\left(1-2 \frac{m}{r}\right)^{-1} d r^{2}-r^{2} d \theta^{2}-r^{2} \sin ^{2}(\theta) d \phi^{2}\right)
$$

From the Schwarzchild solution, it is possible to derive a governing set of differential equations of an orbital system. The differential equations that arise from a solution to the field equations are the equations of geodesic $\mathbb{1}$ motion. The geodesic equations are the EoMs of the system since bodies in space-time travel along time-like geodesics [11]. The geodesic equations that determine the motion of a test particle in a Schwarzchild space-time are (adapted from [14])

\footnotetext{
IA geodesic is the "straightest" possible path in a given space. If one were operating in Euclidian 3 -space, geodesics would be straight lines. If calculations were being done on the surface of a sphere, the geodesics would be great circles.
} 


$$
\begin{aligned}
& 0=\frac{d}{d s}\left[\left(1-\frac{2 m}{r}\right) \frac{d t}{d s}\right] \\
& 0=\frac{d}{d s}\left[-\frac{r^{2}}{c^{2}} \frac{d \theta}{d s}\right]-\frac{1}{2}\left(-\frac{r^{2}}{c^{2}} 2 \sin \theta \cos \theta\right)\left(\frac{d \phi}{d s}\right)^{2} \\
& 0=\frac{d}{d s}\left[-\frac{r^{2}}{c^{2}} \sin ^{2} \theta \frac{d \phi}{d s}\right]
\end{aligned}
$$

Since three-plus-onell coordinates are now being used, it is necessary to derive an independent fourth equation. For this, the first integral (seen in Equation 1.24) of Equations 1.23a, 1.23b, and 1.23c are used [14].

$$
\begin{aligned}
1= & \left(1-2 \frac{m}{r}\right)\left(\frac{d t}{d s}\right)^{2} \\
& -\frac{1}{c^{2}}\left(\left(1-2 \frac{m}{r}\right)^{-1}\left(\frac{d r}{d s}\right)^{2}+r^{2}\left(\frac{d \theta}{d s}\right)^{2}+r^{2} \sin ^{2}(\theta)\left(\frac{d \phi}{d s}\right)^{2}\right)
\end{aligned}
$$

It is possible to parameterize Equations 1.23 and 1.24 using the Classical Orbital Elements (COEs). Using the new relativist expressions for the COEs allows for the reformulation of Kepler's Equation (Equation 1.3) [14], which may be seen in Equation 1.25 [14].

$$
M_{\mathrm{rel}}=E_{\mathrm{rel}}-e \sin E_{\mathrm{rel}}+3 \epsilon\left(1-e^{2}\right) E_{\mathrm{rel}}=\left(t-t_{\circ}\right) \frac{\sqrt{m c^{2}\left(1-e^{2}\right)^{3}}}{p^{\frac{3}{2}}},
$$

where $p$ is the usual semi-parameter seen in the Keplerian formulation. By way of Equation 1.25, it is possible to derive the expression for the relativistic Keplerian period $\mathcal{P}_{\text {rel }}$, shown in Equation 1.26 [14].

$$
\mathcal{P}_{\text {rel }}=\frac{2 \pi p^{\frac{3}{2}}}{\sqrt{m c^{2}\left(1-e^{2}\right)^{3}}}=\frac{2 \pi a^{\frac{3}{2}}}{\sqrt{G M_{\bullet}}}
$$

However, it is important to realize that $\mathcal{P}_{\text {rel }}$ does reduce to the standard Keplerian period. The conclusion that can be drawn from this result is that the period can be predicted using either theory [14].

An orbit propagator can easily be made using Equations 1.25 and 1.26 for simple orbital systems. The consequences of using these equations are that the only true difference seen is in the perceived time length of travel the body moves along the

\footnotetext{
"Three spacial coordinates and one time coordinate
} 
orbit. The propagator is also relatively computationally expensive because of the limited functionality.

An alternate method of deriving EoMs uses field theory. Within this theory, the idea of geodesics is considered to be redundant [18]. Therefore, the EoMs may be determined purely from the field equations (Equation 1.27)

$$
G_{\mu \nu}=0
$$

where $G_{\mu \nu}$ is the Einstein tensor [13]. A linearized metric is utilized when EoMs are being determined by way of this method. The linearized metric takes the form seen in Equation 1.28 .

$$
g_{\mu \nu}=\eta_{\mu \nu}+h_{\mu \nu} \quad \text { or } \quad g^{\mu \nu}=\eta^{\mu \nu}+h^{\mu \nu},
$$

where $h_{\mu \nu}$ and $h^{\mu \nu}$ are approximating terms that are added on to the Minkowskian metric (Equation 1.18) [13].

As the reader has probably noticed by this point, the GTR can be quite complicated and cumbersome to work with. It is possible, and necessary, to make the following two simplifying assumptions [6]. They are: (1) that the central body in a satellite-planet system is the only relativistically significant body, and (2) that the influence of the relativistic forces is so slight that they may be thought of, and treated, as perturbations [6]. Applying these assumptions allows for the modeling of relativistic forces in the same manner that Newtonian gravitational forces and motion are modeled by way of a modified form of Equation 1.8c shown in Equation 1.29 [32].

$$
\ddot{\vec{r}}=-\frac{\mu}{r^{2}} \hat{r}+\vec{a}_{\text {pert }}
$$

The individual relativistic perturbing accelerations that make up $\vec{a}_{\text {pert }}$ will be outlined in detail in Chapter 2. 


\section{Chapter 2}

\section{Orbit Propagation and Perturbation}

The first portion of this chapter is devoted to the discussion of orbit propagation techniques in general. This includes both analytical and numerical methods that were utilized to produce the data that will be presented in Chapter 3. The handling of perturbation terms within the numerical routines will be addressed once the basics of orbit propagation are covered. Finally, the perturbations that are being considered will be discussed.

\subsection{Orbit Propagation}

Orbit propagation techniques can be divided into three main categories. They are: (1) analytical methods, (2) numerical methods, and (3) semianalytical methods. Of these three solution types, analytical techniques and numerical methods were used to generate the data that will be presented in Chapter 3. The following section explains why these methods were chosen, as well as how they were implemented using MatlaB.

\subsubsection{Theoretical Propagation of Equations of Motion}

The analytical propagator is an amalgam of the equations introduced in Section 1.3.1. The structure and flow of the code can be seen in Figure 2.1, and the complete code may be seen in Appendix D. As is seen in Figure 2.1, the inputs are the state vectors for the satellite, the gravitational constant $\mu$, and a time "vector." The time vector is an array of evenly spaced time increments. The propagator uses the time vector to calculate the eccentric anomaly in its overall control loop. 


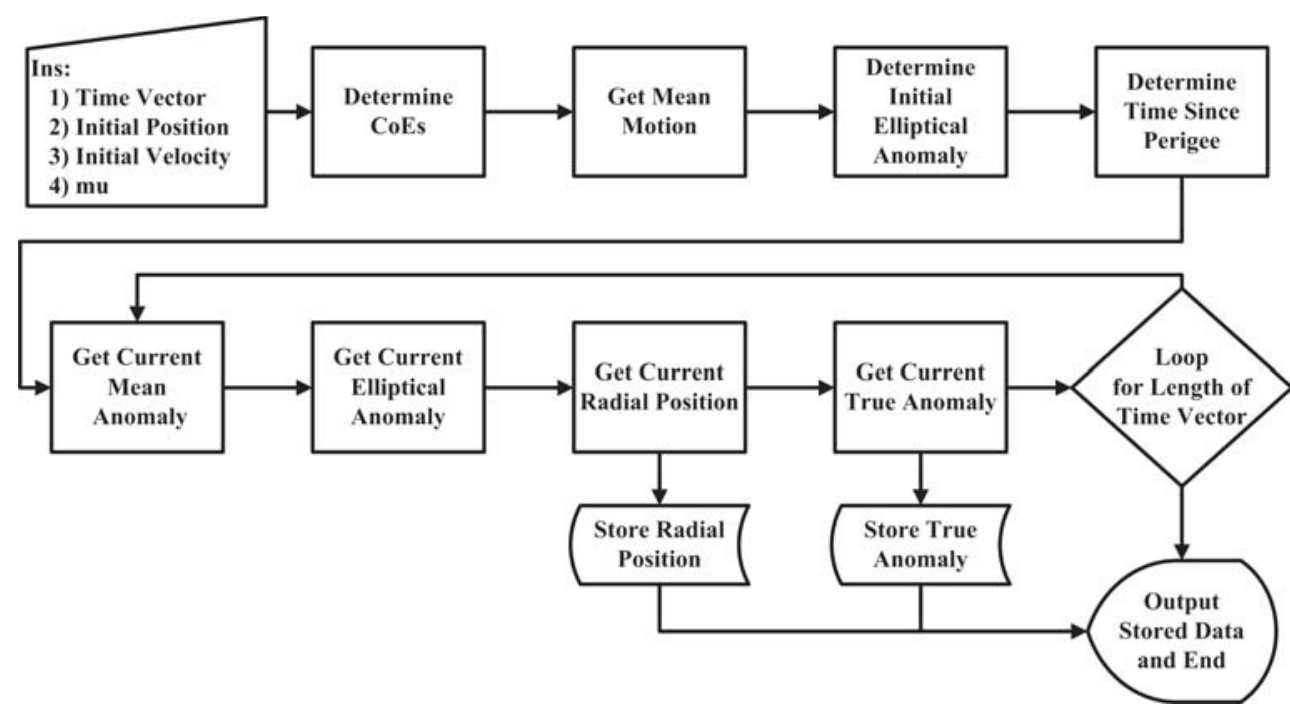

Figure 2.1: Analytical Propagator Architecture

This propagator was used to generate baseline data because it utilizes a closed form solution. However, this propagator is limited in its usefulness. With the formulation that is being used, it is nearly impossible to include perturbation terms. Therefore, it would be a poor choice to use this propagator to generate the data that is presented in Chapter 3.

\subsubsection{Numerical Integration of Equations of Motion}

Even though closed form solutions have been found to the two-body problem, they are only based on the geometry of position data that was collected through observation (Section 1.3.1). In particular, the solution used to create the code, seen in Appendix $\mathrm{D}$, allows for very little modification. The lack of modifiability makes it difficult to add perturbation terms and account for their effects on the geometry of the system. Therefore, it becomes a necessity to look for other modeling options.

Modeling an Ideal Planet-Satellite System As alluded to in Section 1.3.2, Equation 1.8c must be used to develop a state space model of planet-satellite system. 
This system has six states. The state variables, and their derivatives are

$$
\overrightarrow{\mathfrak{z}}=\left\{\begin{array}{c}
x \\
y \\
z \\
\dot{x} \\
\dot{y} \\
\dot{z}
\end{array}\right\} \quad \text { and } \quad \dot{\overrightarrow{\mathfrak{z}}}=\left\{\begin{array}{c}
\dot{x} \\
\dot{y} \\
\dot{z} \\
\ddot{x} \\
\ddot{y} \\
\ddot{z}
\end{array}\right\} \text {, }
$$

where $\mathfrak{z}$ is the state variable. Now that the state variable have been identified, the $A$ matrix must be constructed such that the complete set of state equations takes the form

$$
\dot{\overrightarrow{\mathfrak{z}}}=A \overrightarrow{\mathfrak{z}} .
$$

This is accomplished by looking at Equation 1.8c [32]. The complete state space of the unperturbed system may be seen in Equation 2.3.

$$
\left\{\begin{array}{c}
\dot{x} \\
\dot{y} \\
\dot{z} \\
\ddot{x} \\
\ddot{y} \\
\ddot{z}
\end{array}\right\}=\left[\begin{array}{cccccc}
0 & 0 & 0 & 1 & 0 & 0 \\
0 & 0 & 0 & 0 & 1 & 0 \\
0 & 0 & 0 & 0 & 0 & 1 \\
-\frac{\mu}{r^{3}} & 0 & 0 & 0 & 0 & 0 \\
0 & -\frac{\mu}{r^{3}} & 0 & 0 & 0 & 0 \\
0 & 0 & -\frac{\mu}{r^{3}} & 0 & 0 & 0
\end{array}\right\}\left\{\begin{array}{c}
x \\
y \\
z \\
\dot{x} \\
\dot{y} \\
\dot{z}
\end{array}\right\}
$$

The code used to implement this model in MATLAB may be seen in Appendix E. This setup provides the flexibility for the addition of perturbing terms that are used to model relativistic effects.

MATLAB's ode45 routine was used to integrate this system. It was found that the tolerances on the integration error needed to be tightened through testing with standard and well known orbits. The numerical drift in the system was lowered to the range of machine precision zero* with the increased tolerances. This is important to note since the range of the terms that are being calculated are only slightly above this point.

\subsubsection{Modeling Perturbation Accelerations}

There are two methods for incorporating perturbations into the equations of motion for an orbital system. They are special perturbation techniques and general perturbation techniques (which include semianalytical solutions).

*This has a numerical value of $10 \mathrm{E}-16$ [25]. 


\subsubsection{General Perturbation Techniques}

General perturbation techniques include several different solutions. These are formulated using the Lagrange planetary equations and apply some form of variation of parameters [32]. These methods are not convenient for use with the terms that are being looked at because the perturbations that are being discussed have been formulated in terms of specific force in more recent work [6,28]. The Lagrange planetary form of the Lense-Thirring procession term has been formulated [29]. It would be possible to formulate the rest of the perturbation terms in the form of the Lagrange planetary equations; however, this was not the intent of the work that is being presented.

\subsubsection{Special Perturbation Techniques}

These techniques, and numerical methods in general, are becoming more popular than most analytical techniques as computing power continues to grow [32]. Analytical methods in general tend to be much slower because of the intensity of the necessary calculations. Equation 1.29 is the essence of Crowell's formulation ${ }^{\dagger}$. From Equation 1.29, a new state space could be determined. As one should have expected, the new state space should have the same form as the state space shown in Equation 2.2. The new set of state variables, and their derivatives, are seen in Equation 2.4 [4].

$$
\overrightarrow{\mathfrak{z}}=\left\{\begin{array}{c}
x \\
y \\
z \\
\dot{x} \\
\dot{y} \\
\dot{z}
\end{array}\right\} \quad \text { and } \quad \dot{\vec{z}}=\left\{\begin{array}{c}
\dot{x} \\
\dot{y} \\
\dot{z} \\
\ddot{x}-\ddot{x}_{\text {pert }} \\
\ddot{y}-\ddot{y}_{\text {pert }} \\
\ddot{z}-\ddot{z}_{\text {pert }}
\end{array}\right\}
$$

The matrix shown in Equation 2.3 stays the same. This fact allows Crowell's formulation is flexible enough to use the same code with several different perturbing terms. The only requirement on the perturbing terms is that they must be expressible in terms of a vector-valued specific force function [32]. Hence, the modified state

†This should not be confused with Crowell's method, which is a method of numerical integration $[32]$. 
space is shown in Equation 2.5.

$$
\left\{\begin{array}{c}
\dot{x} \\
\dot{y} \\
\dot{z} \\
\ddot{x} \\
\ddot{y} \\
\ddot{z}
\end{array}\right\}=\left[\begin{array}{cccccc}
0 & 0 & 0 & 1 & 0 & 0 \\
0 & 0 & 0 & 0 & 1 & 0 \\
0 & 0 & 0 & 0 & 0 & 1 \\
-\frac{\mu}{r^{3}} & 0 & 0 & 0 & 0 & 0 \\
0 & -\frac{\mu}{r^{3}} & 0 & 0 & 0 & 0 \\
0 & 0 & -\frac{\mu}{r^{3}} & 0 & 0 & 0
\end{array}\right]\left\{\begin{array}{c}
x \\
y \\
z \\
\dot{x} \\
\dot{y} \\
\dot{z}
\end{array}\right\}+\left\{\begin{array}{c}
0 \\
0 \\
0 \\
\ddot{x}_{\text {pert }} \\
\ddot{y}_{\text {pert }} \\
\ddot{z}_{\text {pert }}
\end{array}\right\}
$$

MATLAB's ode45 routine was used to integrate this system. The code used to implement this state space may be seen in Appendices F and G.

\section{$2.2 \quad$ Orbit Perturbation}

The special perturbation technique and Crowell's formulation were chosen to produce the numerical results shown in Chapter 3 because of the versatility that this combination provides for modeling several different perturbations in rapid succession. The form of the relativistic perturbing terms used in the numerical simulations are based on those presented in Applied Orbit Perturbation and Maintenance [6]. This section will introduce the acceleration terms that were used during the numerical simulations. How the perturbations arise and manifest in the Earth-satellite system (or for certain perturbations - the Earth-Sun-satellite system) will also be discussed. All of the perturbation terms were embedded into one MATLAB function for ease of use. This function may be seen in Appendix J.

\subsubsection{Central Body Terms}

As mentioned in Chapter 1, the gravitational field and the matter embedded within the field are both equally important in the GTR. However, in order to have an accurate picture of how a gravitational field emanates from a central body, there must be an acceptable model for the body itself [29]. The central body shape effects are modeled in the relativistic framework as a combination of two perturbation terms [28]. They are (1) the spherical perturbation term and (2) the relativistic oblateness term.

The spherical body perturbation term may be seen in Equation 2.6. This term is analogous to the result produced by the Schwarzschild solution because they both provide the acceleration of a test particle around a spherically symmetric central body $[10,28,29]$.

$$
\vec{a}_{\mathrm{ST}}=\frac{\mu_{\oplus}}{c^{2} r^{3}}\left\{\left[2(\gamma+\beta) \frac{\mu_{\oplus}}{r}-\gamma(\dot{\vec{r}} \cdot \dot{\vec{r}})\right] \vec{r}+2(1+\gamma)(\vec{r} \cdot \dot{\vec{r}}) \dot{\vec{r}}\right\}
$$


Earth is not spherically symmetric [31,32]. The shape of Earth is technically an oblate spheroid. In non-relativistic theory, the Earth's oblateness is modeled using derivatives of the zonal harmonic functions [32]. The most prominent of these is second zonal harmonic term [4], which produces what is commonly referred to as the $\mathbf{J}_{\mathbf{2}}$-term. The final perturbation acceleration for the $\mathbf{J}_{\mathbf{2}}$-term takes the form seen in Equation 2.7 [32].

$$
\vec{a}_{\mathrm{J} 2}=-\frac{3 \mathbf{J}_{2} \mu R_{\oplus}^{2}}{2 r^{5}}\left\{\begin{array}{l}
r_{i}\left(1-\frac{5 r_{k}^{2}}{r^{2}}\right) \\
r_{j}\left(1-\frac{5 r_{k}^{2}}{r^{2}}\right) \\
r_{k}\left(3-\frac{5 r_{k}^{2}}{r^{2}}\right)
\end{array}\right\},
$$

where $\mathbf{J}_{\mathbf{2}}$ is the Earth's oblateness factor and $R_{\oplus}$ is Earth's average radius. However, this term does not take into account the relativistic effects acting on the system [29].

Therefore, it is necessary for the derivation of appropriate oblateness terms that accurately describe the Earth's shape within the relativistic theory [29]. The relativistic oblateness term seen in Equation 2.8 was derived in [29].

$$
\begin{aligned}
\vec{a}_{\mathrm{RJ} 2}= & \mathbf{J}_{2} \frac{\mu_{\oplus}}{c^{2} r^{3}}\left(\frac{a_{\oplus}}{r}\right)^{2}\{\cdots \\
& 2(\gamma+\beta) \frac{\mu_{\oplus}}{r}\left[\begin{array}{l}
x\left(2-9\left(\frac{z}{r}\right)^{2}\right) \\
y\left(2-9\left(\frac{z}{r}\right)^{2}\right. \\
z\left(5-9\left(\frac{z}{r}\right)^{2}\right.
\end{array}\right]-\frac{3}{2}\left(\frac{\dot{\vec{r}} \cdot \dot{\vec{r}}}{r}\right)\left[\begin{array}{l}
x\left(1-5\left(\frac{z}{r}\right)^{2}\right) \\
y\left(1-5\left(\frac{z}{r}\right)^{2}\right) \\
z\left(3-5\left(\frac{z}{r}\right)^{2}\right)
\end{array}\right] \cdots \\
& \left.+3(1+\gamma)\left[\left(1-5\left(\frac{z}{r}\right)^{2}\right)(\vec{r} \cdot \dot{\vec{r}})+2 z \dot{z}\right] \dot{\vec{r}}\right\}
\end{aligned}
$$

The relativistic oblateness term may be regarded as an updated version of the standard oblateness term because Equation 2.7 was the starting point to derive the necessary relativistic correction [29]. This fact is evident because on comparison of the two equations, it is possible to see that the standard oblateness term is bound up within Equation 2.8.

\subsubsection{Lense-Thirring and Rotational Energy Terms}

The Lense-Thirring perturbation term is referred to as a gravitomagnetic effect and arises from angular momentum of the central body [28]. This manifests itself by "dragging" a reference frame along an orbit in the direction of the the central body's rotation [26]. In other words, the Lense-Thirring effect forces the orbit to precess around the central body in the direction of the central body's rotation. Therefore, it 
is no surprise that this term is more commonly known as frame dragging. The frame dragging effect may be visualized as seen in Figure 2.2b. The white arrow in Figure

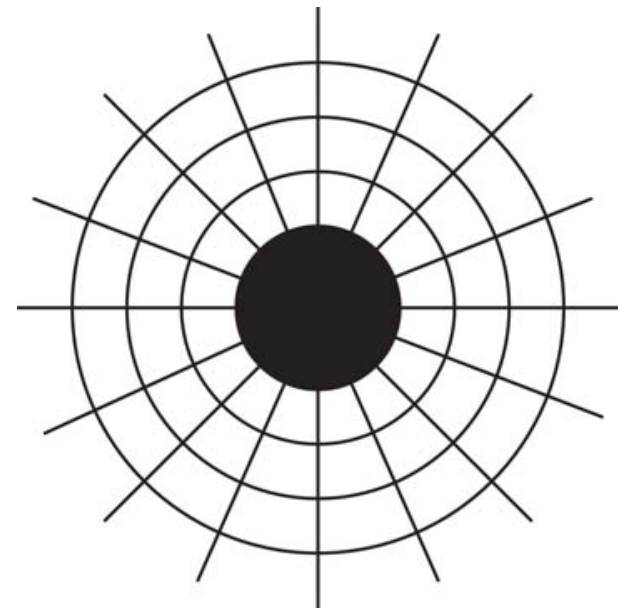

(a) Non-Rotating Central Body

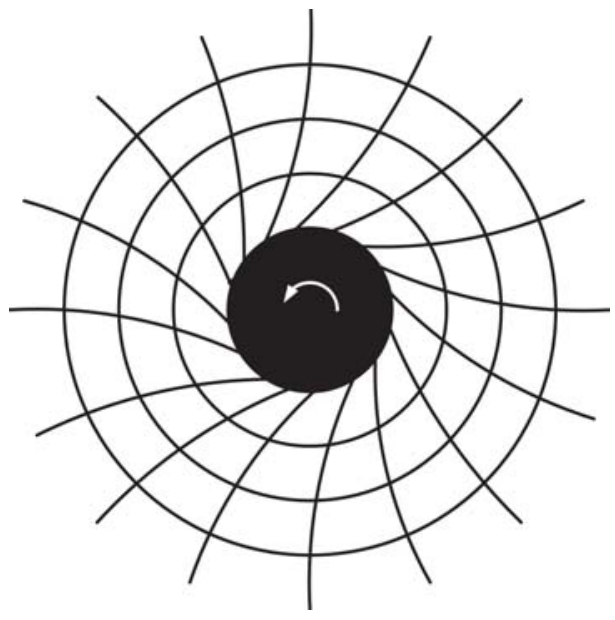

(b) Rotating Central Body

Figure 2.2: Two Dimensional Space-Times

$2.2 \mathrm{~b}$ shows the direction of rotation of the central body. The non-rotating space-time seen in Figure 2.2a has been added for comparison purposes. (Note: Figure 2.2 is a sketch and not drawn to scale.)

The expression that gives this perturbations contribution is seen in Equation 2.9. As is seen in Equation 2.9, the expression includes a specific angular momentum term $\overrightarrow{J^{*}}$ (which may be seen in Appendix C) and the Lense-Thirring parameter $L$.

$$
\vec{a}_{\mathrm{LT}}=L(1+\gamma) \frac{\mu_{\oplus}}{c^{2} r^{3}}\left[\frac{3}{r^{2}}\left(\vec{r} \cdot \overrightarrow{J^{*}}\right)(\vec{r} \times \dot{\vec{r}})+\left(\dot{\vec{r}} \times \overrightarrow{J^{*}}\right)\right]
$$

Within the GTR framework $L$ may be taken to equal 1.0 [28]. Using this as a simplifying assumption, the classical form of the Lense-Thirring term is found if the expression in Equation 2.9 is expanded while imposing this limit on $L$. The classical form of the Lense-Thirring acceleration is seen in Equation 2.10 as shown by [28].

$$
\vec{a}_{\mathrm{LT}}=\frac{\nu}{r^{3}}\left\{\begin{array}{c}
\dot{y}+3 \frac{z}{r^{2}}(y \dot{z}-z \dot{y}) \\
-\dot{x}+3 \frac{z}{r^{2}}(z \dot{x}-x \dot{z}) \\
3 \frac{z}{r^{2}}(x \dot{y}-y \dot{x})
\end{array}\right\} \quad \text { where } \quad \nu=\frac{2}{5}(1-\gamma) \frac{\mu_{\oplus} R_{\oplus}^{2} \Omega_{\oplus}}{c^{2}}
$$

Equation 2.10 was used to create the data that is presented in Chapter 3.

The Lense-Thirring term does not give the entire picture of frame dragging because there are accelerations due to a term that arises completely from energy analysis [28]. 
This term is known as the rotational energy perturbation acceleration and may be seen in Equation 2.11 [6,28].

$$
\vec{a}_{\mathrm{RE}}=\frac{3}{14} T_{\oplus}^{*}(1+\gamma) \frac{\mu_{\oplus}}{c^{2} r^{3}}\left(\frac{a_{\oplus}}{r}\right)^{2}\left\{\left(1-5\left(\frac{z}{r}\right)^{2}\right) \vec{r}+2\left(\vec{r} \cdot \hat{\Omega}_{\oplus}\right) \hat{\Omega}_{\oplus}\right\}
$$

It is difficult to find any information on this term, and its effects, because neither of them have been discussed in the literature [28]. However, the work of Peterson (see reference [28]) has shown that the rotational energy term does contribute small perturbing forces.

It is interesting that $\vec{a}_{\text {RelJ2 }}$ and $\vec{a}_{\mathrm{NRG}}$ have the same form. The consequence of this is that these two terms' contributions cannot be uniquely separated when looking at observational data [28]. To combat this, Peterson was able to back out an effective $\mathbf{J}_{2}$ parameter from the data that was collected. Since the aim of the work being presented within this thesis is to characterize individual perturbations ${ }^{\ddagger}$, it was deemed that the current accepted value of $\mathbf{J}_{\mathbf{2}}$ (seen in Appendix $\mathrm{C}$ ) would be sufficient for the computational purposes at hand.

\subsubsection{Geodesic Precession Term}

Geodesic precession has its roots very early on in the GTR. It was first proposed by Willem de Sitter in a series of papers written for Monthly Notices of the Royal Astronomical Society [7-9]. The derivation that de Sitter presents results in the expression of the planetary orbit equation for an elliptical orbit with a moving perihelion point (e.g. Mercury), as well as the expression for the rate of precession [7].

The lunar motion problem was then approached by de Sitter using the aforementioned work as a starting point [8]. This is indeed a more difficult problem because there are now three interactions to be concerned with: Sun-Moon, Sun-Earth, and Earth-Moon [23]. This type of problem is a general relativistic three-body problem [32]. However, it lays the groundwork nicely for a simplification to a restricted relativistic three-body problem by using two simplifying assumptions. These two simplifying assumptions are [32]:

1. The two primary bodies orbit in a circular orbit around their center of mass.

2. The satellite has negligible mass compared to that of the primary bodies.

The Earth and Sun are the two primary bodies. An Earth bound satellite is the third body for the work being presented in this thesis. Since this system meets the

\footnotetext{
${ }_{\ddagger}^{\ddagger}$ Each perturbation was integrated separately. Therefore, there were no interactions with other terms.
} 
requirements of the restricted three-body problem, it may be treated as such.

The form of the geodesic precession acceleration that was used to create the data presented in Chapter 3 may be seen in Equation 2.12 [6].

$$
\vec{a}_{\mathrm{GP}}=-\frac{\mu_{\odot}}{c^{2} R_{\oplus / \odot}^{3}}(1+2 \gamma)\left(\dot{\vec{R}}_{\oplus / \odot} \times \vec{R}_{\oplus / \odot}\right) \times \dot{\vec{r}}
$$

As should have been expected, Equation 2.12 contains terms for the Sun, Earth, and satellite. Specifically, the gravitational parameter of the Sun $\mu_{\odot}$, the relative position and velocity vectors of the Earth with respect to the Sun, $\vec{R}_{\oplus / \odot}$ and $\dot{\vec{R}}_{\oplus / \odot}$ respectively, and the velocity vector of the satellite with respect to the Earth $\dot{\vec{r}}$.

With the amount of information needed to compute this term, great care had to be taken in order to guarantee accurate results. All calculations for this term, as well as the previously mentioned terms, were done in the Earth-fixed coordinate frame. It should also be noted that the Earth's position and velocity data were generated using the HORIZONS system ${ }^{\S}$, which is maintained by the Solar System Dynamics Group of the Jet Propulsion Laboratory (JPL) $\mathbb{I}$. The JPL is a combined effort between the California Institute of Technology" and NASA**.

\footnotetext{
$\S_{\text {http://ssd.jpl.nasa.gov/?horizons }}$

$\mathbb{I}_{\text {http: //www.jpl.nasa.gov/ }}$

"http://www.caltech.edu/

**http://www.nasa.gov/
} 


\section{Chapter 3}

\section{Numerical Results of Relativistic Perturbations}

This chapter contains a discussion of the effects that arise from the implementation of the relativistic oblateness perturbation on two different orbit cases. The data presented includes orbital parameter differences plots as well as specific force magnitudes. This is followed by a comparison of the relativistic oblateness perturbation to the standard oblateness perturbation developed in the classical theory. The data for the orbital parameters and specific force magnitudes of the two test orbit cases of the aforementioned relativistic perturbations may be seen in Appendices K and L.

\subsection{The Test Case Orbits}

The orbits that were used as test cases were defined by the usual Classical (or Keplerian) Orbital Elements (COE). The standard case is a non-equatorial elliptical orbit with medium eccentricity. The special case that was tested was a Molniya orbit. The Molniya orbit was determined by Russian scientist to provide constant coverage over Russian air space for communication satellites [32]. To accomplish this, the orbit had to have extremely high angle of inclination and eccentricity. The complete set of classical orbital elements for both test orbits may be seen in Table 3.1. The periods of the test orbits may be seen in Table 3.2. In the Keplerian theory, these parameters have static values $[4,32]$. However, this is not the case when perturbations are being considered. The modulation of the classical orbital elements will be discussed in the following sections. 
Table 3.1: Orbital Parameters for Test Orbit Cases

\begin{tabular}{c|c|c|c} 
Orbital Parameter & Standard Value & Molniya Value & Units \\
\hline \hline$\theta_{\circ}$ & 28.0000 & 28.0000 & degrees \\
\hline$e_{\circ}$ & 0.4500 & 0.7000 & n/a \\
\hline$a_{\circ}$ & $1.34147272 \mathrm{E} 4$ & $2.66102228 \mathrm{E} 4$ & kilometers \\
\hline$p_{\circ}$ & $1.06982450 \mathrm{E} 4$ & $1.35712136 \mathrm{E} 4$ & kilometers \\
\hline$i_{\circ}$ & 32.0000 & 63.9000 & degrees \\
\hline$\omega_{\circ}$ & 18.0000 & 0.0000 & degrees \\
\hline$\Omega_{\circ}$ & 24.0000 & 0.0000 & degrees
\end{tabular}

Table 3.2: Periods of Test Orbits

\begin{tabular}{|c|c|c|}
\cline { 2 - 3 } \multicolumn{1}{c|}{} & \multicolumn{2}{c|}{ Orbit Cases } \\
\cline { 2 - 3 } \multicolumn{1}{c|}{} & Standard Orbit & Molniya Orbit \\
\hline $\begin{array}{c}\text { Orbital Period } \\
(\mathcal{P}) \\
(\text { hours })\end{array}$ & $\approx 4.2952$ & 12.0000 \\
\hline
\end{tabular}

\subsection{Relativistic Oblateness Orbital Element Data}

The effects of the relativistic oblateness perturbations on the standard orbit and the Molniya orbit will be discussed in the following sections. The graphs of the orbital parameter data that were generated using the code presented in Chapter 2.

The difference plots were generated by taking the data from the desired perturbation and subtracting out the data produced by integrating the orbit without perturbation. Each case has a figure that contains the angular parameter difference plots and a separate figure for the distance difference plots - note that the unitless term is grouped with the distance terms. It is possible to classify the modulations experienced by the orbital elements into three classes [4]. They are: (1) short term periodic, (2) long term periodic, and (3) secular (adapted from [4]). Short and long term periodic modulations are somewhat nebulous terms because they are based on the individual characteristics of a difference plot. A secular variation is a modulation that has a consistent trend over a reasonably long period of time. 


\subsubsection{The Standard Case}

\subsubsection{Periodic Orbital Elements}

There are two orbital elements that exhibit periodicity. They are the true anomaly $\theta$ and the magnitude of spacecraft's position vector $r$ which are seen in Figures 3.1 and 3.2 respectively. Both difference plots show that the behavior of these elements is short term periodic. This periodicity of these difference plots suggests that these elements experience phasing. When the graphs of these elements are on or cross the zero line, they have equivalent values of the unperturbed orbit at that time. The farther that the graph moves away from the zero line, the more out of phase the element is at that particular time.

Both orbits, while exhibiting a strong periodic behavior, also show secular characteristics as well. It is obvious on examination of Figure 3.1 that the true anomaly difference increases with time. However the difference plot of the spacecraft position vector's magnitude, seen in Figure 3.2, shows a full reversal. This full reversal implies that this difference quantity has a lead-lag relationship with the unperturbed orbit.
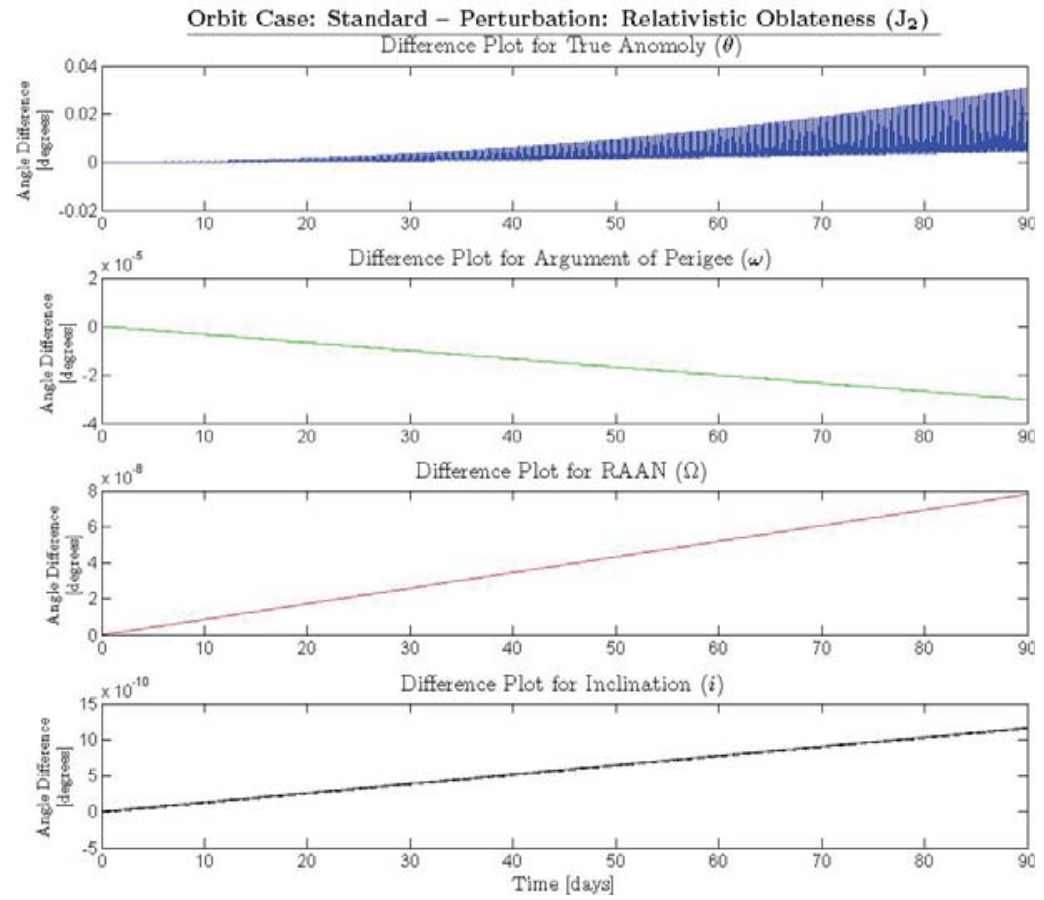

Figure 3.1: Standard Orbit Angular Differences for Relativistic Oblateness versus Time 

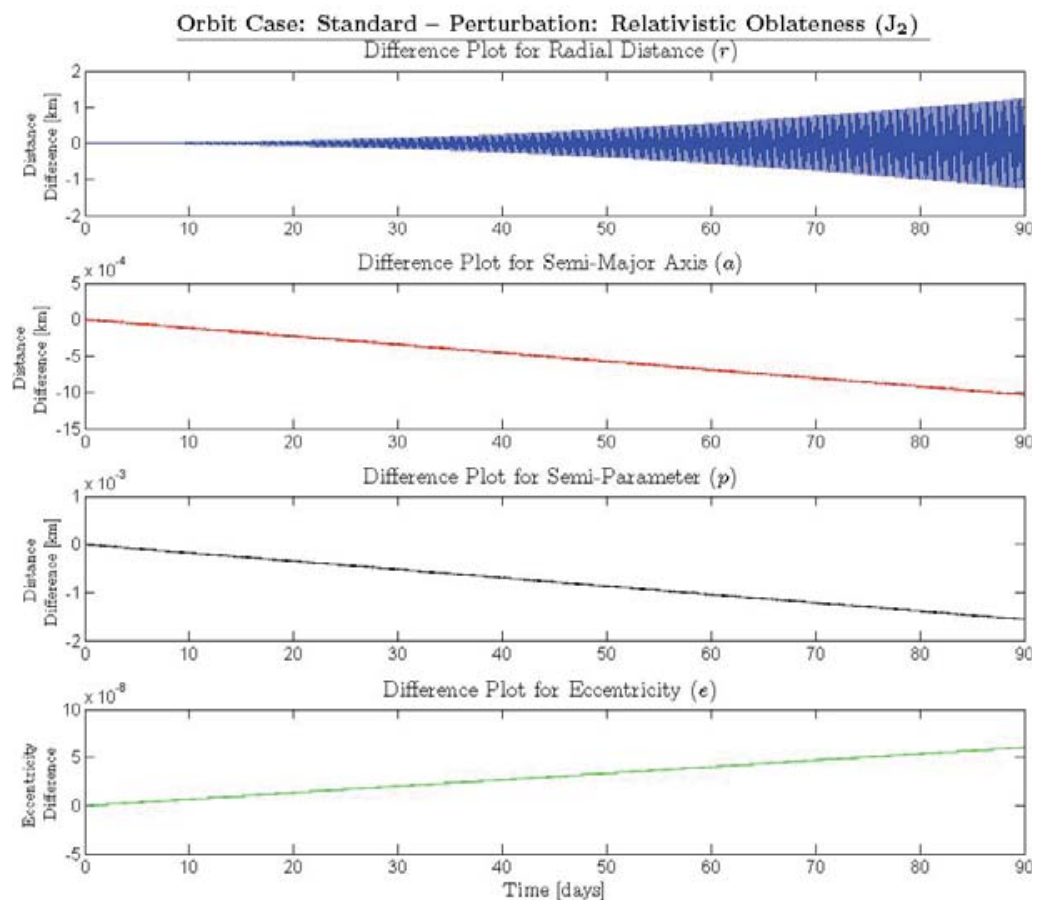

Figure 3.2: Standard Orbit Distance Differences for Relativistic Oblateness versus Time

\subsubsection{Secular Orbital Elements}

The remainder of the orbital elements displayed in Figures 3.1 and 3.2 exhibit predominately secular behavior. On a short time scale, all of these elements do show some short term periodicity. However, the short term periodicity is negligible in effect compared to the secular modulation. The trends of these elements are summarized in Table 3.3.

Table 3.3: Secular Element Trends for Relativistic Oblateness

\begin{tabular}{c|c} 
Element & Trend \\
\hline \hline$\omega$ & Decreasing \\
\hline$\Omega$ & Increasing \\
\hline$i$ & Increasing \\
\hline$a$ & Decreasing \\
\hline$p$ & Decreasing \\
\hline$e$ & Increasing
\end{tabular}




\subsubsection{Molniya Orbit Case}

\subsubsection{Periodic Orbit Elements}

The Molniya orbit has three elements that exhibit periodic behavior. They are the true anomaly $\theta$, the angle of inclination $i$, and the magnitude of the spacecraft position vector $r$. The angle of inclination is the only true periodic element, which exhibits both short and long term periodicity.

The difference plot of the true anomaly $\theta$, shown in Figure 3.3, presents with both short term periodic trends and a secular component. The secular component is easily noted because of the steady increase of the difference. It is also evident that this parameter phases because the difference between the perturbed and unperturbed orbits does go to zero frequently.

The difference plot of the magnitude of the spacecraft position vector, seen in Figure 3.4, does show the same type of phasing. This phasing is evident because the difference between the perturbed and unperturbed orbits does go to zero. Even though this plot does exhibits increasing phase, it is not a true secular variation.
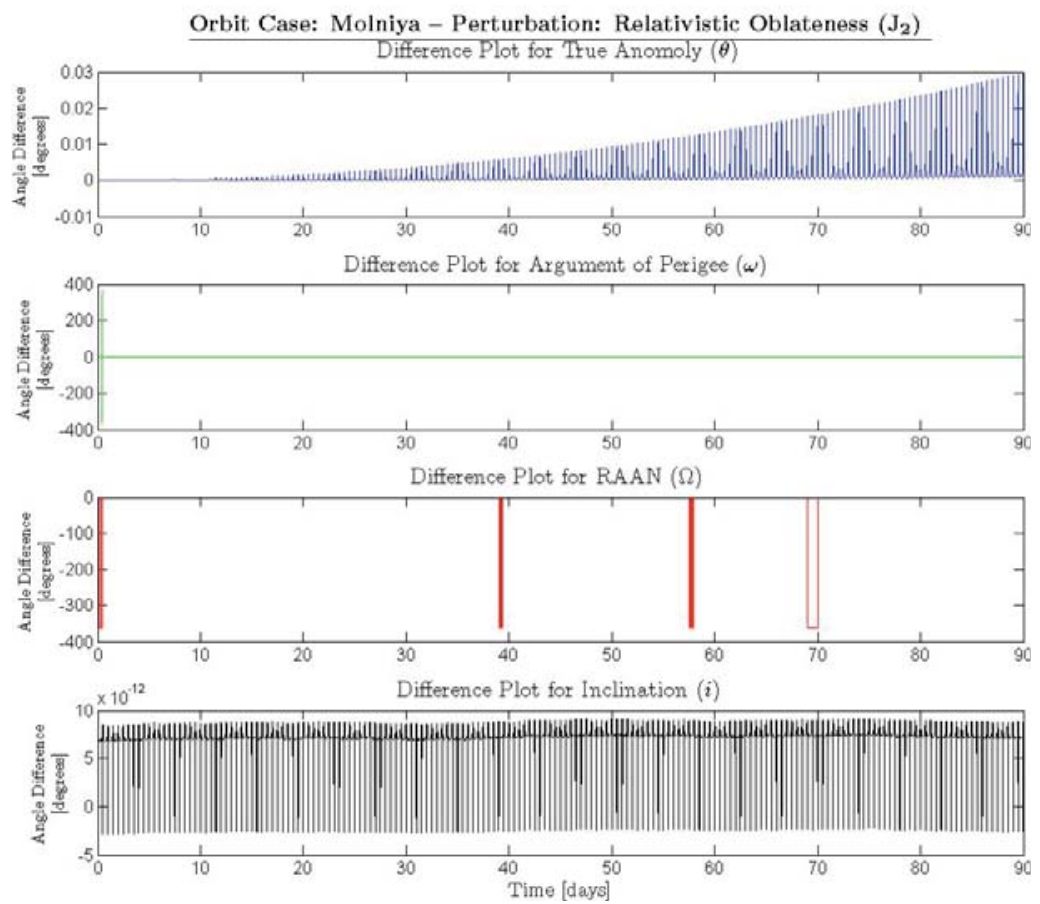

Figure 3.3: Molniya Orbit Angular Differences for Relativistic Oblateness versus Time 

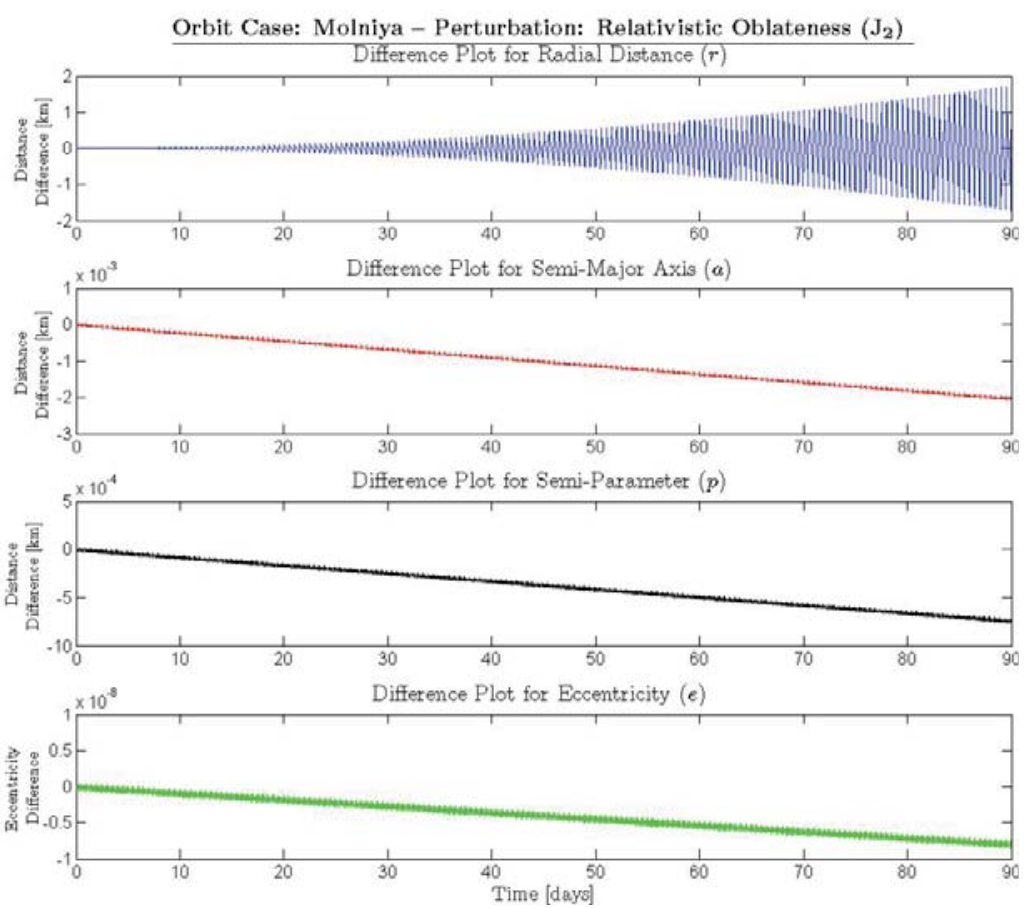

Figure 3.4: Molniya Orbit Distance Differences for Relativistic Oblateness versus Time

\subsubsection{Secular Orbit Elements}

The trends of the purely secular elements for the Molniya orbit are shown in Table 3.4. It is obvious, based on the difference plots contained in Figure 3.4, that the semimajor axis $a$, the semi-parameter $p$, and the eccentricity $e$ are all decreasing further from their values in the unperturbed orbit. However, the argument of periapsis $\omega$ and longitude of the ascending node (a.k.a. RAAN) $\Omega$ are both indeterminate.

Table 3.4: Trends of the Secular Elements for the Molniya Orbit

\begin{tabular}{c|c} 
Element & Trend \\
\hline \hline$a$ & Decreasing \\
\hline$p$ & Decreasing \\
\hline$e$ & Decreasing \\
\hline$\Omega$ & Indeterminate \\
\hline$\omega$ & Indeterminate
\end{tabular}




\subsection{Effects of Standard Oblateness on Orbital El- ements}

\subsubsection{Standard Orbit}

Periodic Elements As seen in Figures 3.5 and 3.6, there are six elements that exhibit periodicity. Four of these six manifest as having both short and long term periods. They are $i, a, p$, and $e$. This periodic behavior is what should be expected due to the relationship between these elements and that only odd numbered zonal harmonics produce secular variations [4]. It is also possible to prove that these elements should experience periodic modulation based on their relationship to the spacecraft angular momentum along the orbit path [4].

The true anomaly exhibits both short term periodicity, but also has a secular component as is seen in Figure 3.5 by the downward trend. This implies that the perturbed orbit lags compared to the unperturbed orbit. The magnitude of the spacecraft position vector, seen in Figure 3.6, shows short term periodic behavior. However, it seems to come to a steady state without further increase in the peak-topeak value of the difference plot.
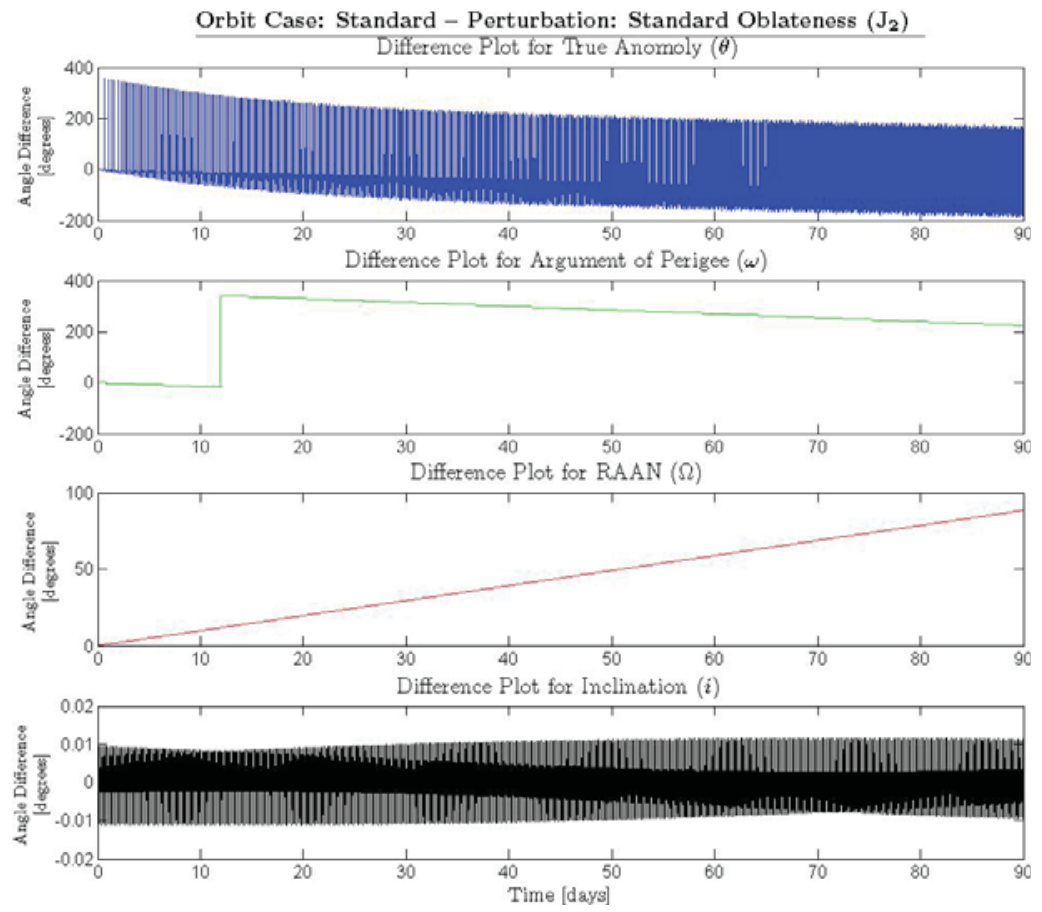

Figure 3.5: Standard Orbit Angular Differences for Standard Oblateness Pert. versus Time 

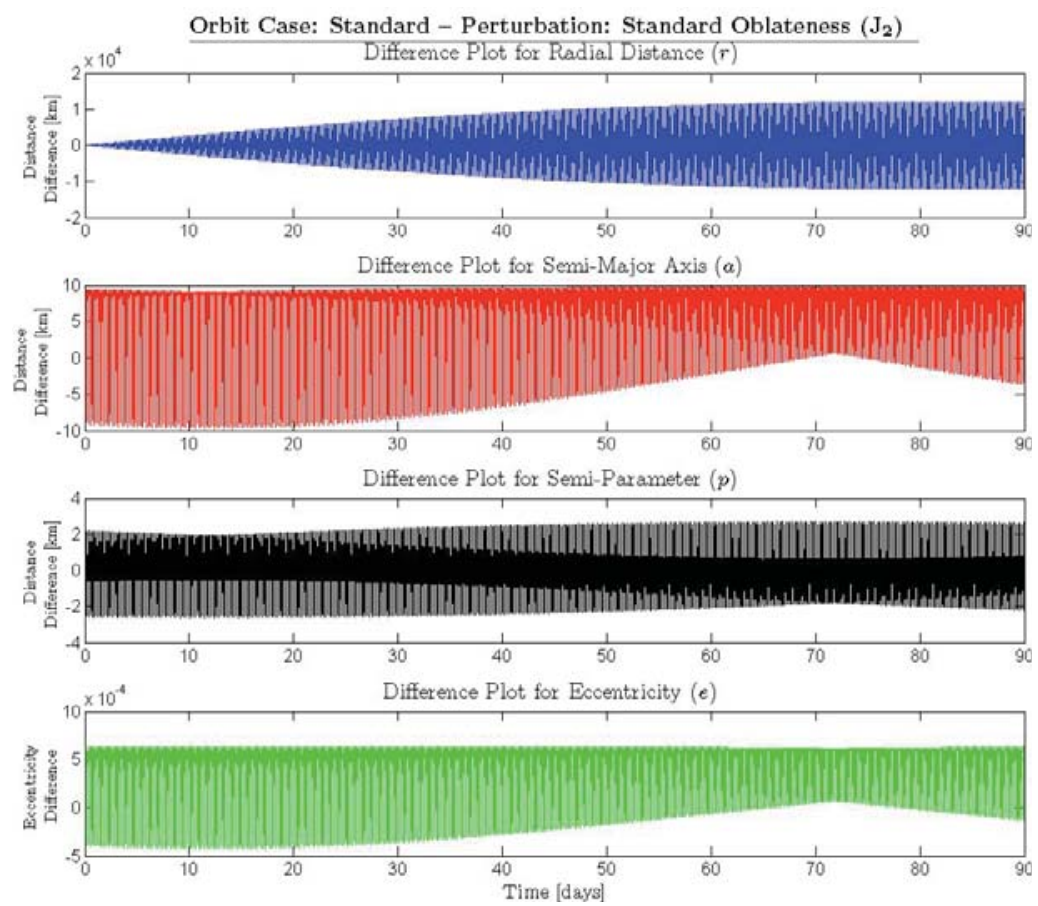

Figure 3.6: Standard Orbit Distance Differences for Standard Oblateness Pert. versus Time

Secular Elements There are only two truly secular elements caused by this perturbation when applied to this orbit case. They are $\Omega$ and $\omega$. It is obvious during the inspection of Figure 3.5 that $\Omega$ has an upward trend and $\omega$ is trending downward. The fact that neither of these two elements contain a periodic component is in agreement with the solution results presented by Blitzer [4].

\subsubsection{Molniya Orbit Results}

Periodic Elements As with the standard orbit case, six of the elements display periodic effects. They are $\theta, i, r, a, p$, and $e$. All of these elements exhibit signs of short period modulation. Two of the elements, $r$ and $\theta$, display other behaviors as well. The true anomaly $\theta$ has a secular component that has a decreasing trend which is easily seen in Figure 3.7. The peak-to-peak value of the difference plot of $r$ also increases continually. 

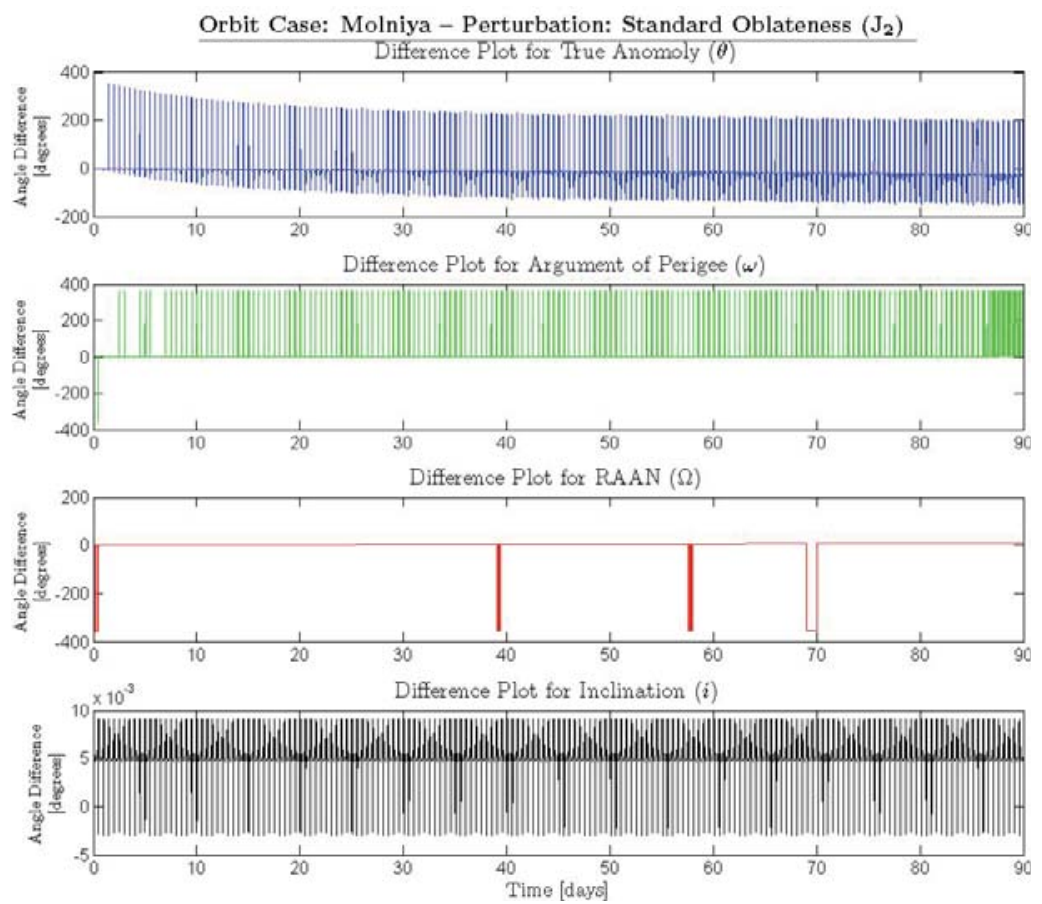

Figure 3.7: Molniya Orbit Angular Differences for Standard Oblateness Pert. versus Time
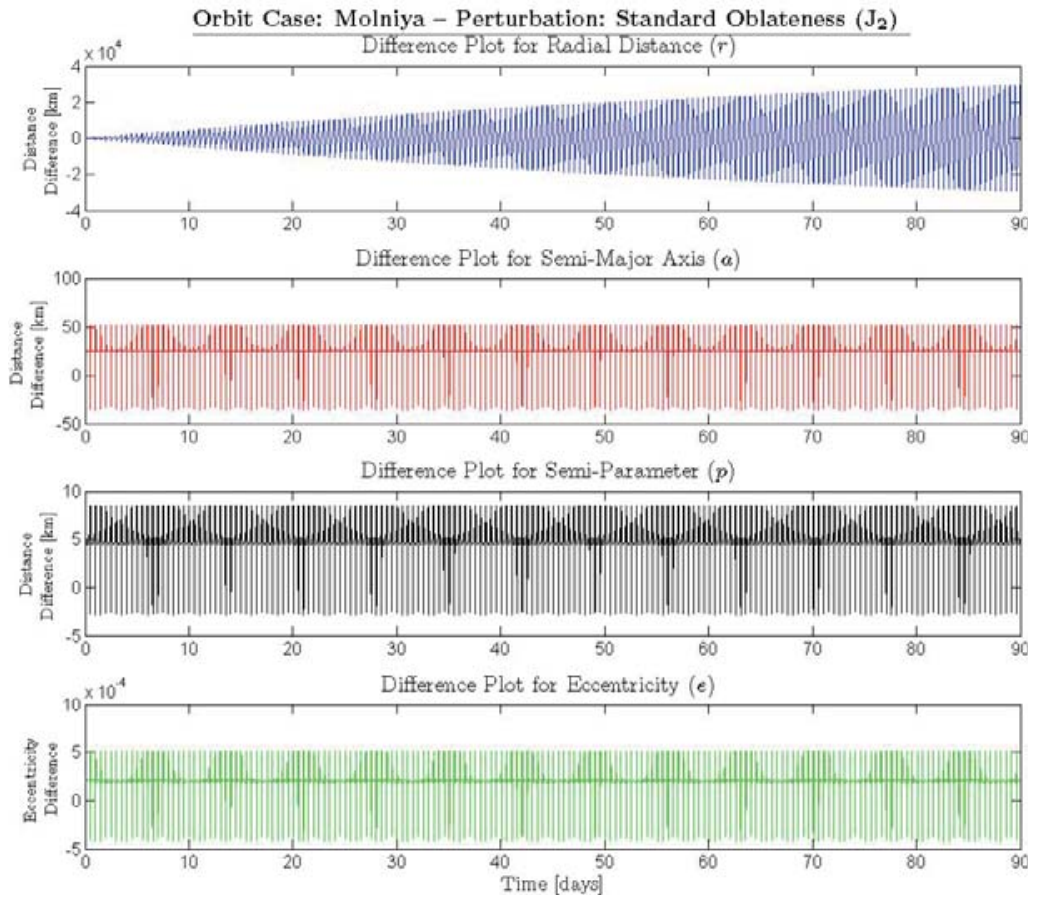

Figure 3.8: Molniya Orbit Distance Differences for Standard Oblateness Pert. versus Time 
Secular Elements The secular elements for this perturbation on this orbit case should be $\Omega$ and $\omega$ [4]. However, both of these difference plots, seen in Figure 3.7, are indicative of secular modulation. The difference plots of these two elements contain in Figure 3.7 imply a zero-point fluctuation of the aforementioned elements.

\subsection{Discussion of Orbital Element Data}

The data presented in Figures 3.1 to 3.8 has been summarized in Tables 3.5 and 3.6. Table 3.5 displays the type of modulation (and trend where applicable) exhibited by each of the elements under both standard and relativistic oblateness. The minimum, maximum, mean, and median values of the data contained in the aforementioned tables are shown in Table 3.6.

\subsubsection{Data Trends Analysis}

Table 3.5: Summary of Data Trends

\begin{tabular}{|c|c|c|c|c|c|}
\hline & & \multicolumn{2}{|c|}{ Standard Oblateness } & \multicolumn{2}{|c|}{ Relativistic Oblateness } \\
\hline & & $\begin{array}{l}\text { Standard } \\
\text { Orbit }\end{array}$ & $\begin{array}{c}\text { Molniya } \\
\text { Orbit }\end{array}$ & $\begin{array}{l}\text { Standard } \\
\text { Orbit }\end{array}$ & $\begin{array}{c}\text { Molniya } \\
\text { Orbit }\end{array}$ \\
\hline \multirow{2}{*}{$\theta$} & Variation & Sec. and Per. & Sec. and Per. & Sec. with Per. & Sec. with Per. \\
\hline & Trend & Decreasing & Decreasing & Increasing & Increasing \\
\hline \multirow{2}{*}{$a$} & Variation & Periodic & Periodic & Secular & Secular \\
\hline & Trend & $n / a^{-\cdots .}$ & $n / a^{--1}$ & Decreasing & Decreasing \\
\hline \multirow{2}{*}{$e$} & Variation & Periodic & Periodic & Secular & Secular \\
\hline & Trend ${ }^{-}$ & $\mathrm{n} / \mathrm{a}^{\cdots}$ & $\mathrm{n} / \mathrm{a}^{\cdots}$ & Increasing & Decreasing \\
\hline \multirow{2}{*}{$p$} & Variation & Periodic & Periodic & Secular & Secular \\
\hline & Trend & n/a & $\mathrm{n} / \mathrm{a}^{-}$ & Decreasing & Decreasing \\
\hline \multirow[b]{2}{*}{$\Omega$} & Variation & Secular & Indeterminate & Secular & Indeterminate \\
\hline & Trend & Increasing & Indeterminate- & Increasing & Indèterminate- \\
\hline \multirow{2}{*}{$\omega$} & Variation & Secular & Indeterminate & Secular & Indeterminate \\
\hline & Trend & Decreasing & Indeterminate & Decreasing & Indeterminate \\
\hline \multirow[b]{2}{*}{$i$} & Variation & Periodic & Periodic & Secular & Periodic \\
\hline & Trend & $\mathrm{n} / \mathrm{a}^{\cdots}$ & $\mathrm{n} / \mathrm{a}^{-\cdots}$ & Increasing & n $7 a^{\cdots}$ \\
\hline
\end{tabular}

There is indeed a difference between the standard and relativistic oblateness perturbations based on the modulations and trends seen in Table 3.5. There are also striking similarities between the trends. Examples of these similarities are the true anomaly $\theta$, the RAAN $\Omega$, and argument of periapsis $\omega$. The determination of $\Omega$ and $\omega$ have been labeled as "Indeterminate" because of the difficulties of the integration with zero-point fluctuations. With that aside, it is possible to see that the three previously mention elements have the same type of modulation in each orbit case for their 
respective perturbations. The trends for $\Omega$ and $\omega$ remain the same for both perturbations. However, there is a change in the trends exhibited by the true anomaly. The standard oblateness perturbation forces induce a decrease in this quantity, whereas the relativistic oblateness perturbation induces an increase.

The same pattern is seen in the semi-major axis $a$ and semi-parameter $p$. Both of these elements have been shown to only exhibit purely periodic modulation under the standard oblateness perturbation [4]. When the relativistic oblateness perturbation is applied it is seen that both of these elements modulate in a secular fashion with a decreasing trend.

Eccentricity's, $e$, modulation type switches to secular with the implementation of the relativistic perturbation in the same manner as the modulation type of $a$ and $p$ in the preceding paragraph. However, there is a unique discrepancy of how the relativistic oblateness perturbation manifest in the standard orbit compared to how the perturbation manifests in the Molniya orbit. The relativistic oblateness perturbation affects the test orbits' eccentricity in the opposite manner - i.e. the standard orbit's eccentricity increases while the eccentricity of the Molniya orbit decreases. Nothing in the current literature suggests a reason for this disparity between the effects of the different oblateness perturbations on the eccentricity. One possible explanation for this phenomena is that there is an unstable equilibrium configuration of the orbit somewhere between the eccentricities of the two test orbits. However, this may not be completely true because there is also an unusual effect observed in the inclination.

The angle of inclination $i$ with the inclusion of the standard oblateness perturbation has the same effect on each of the orbit cases. In both trial orbits, $i$ experienced periodic modulation. This is in agreement with the proof provided by Blitzer [4]. This is the same type of variation that is exhibited with the Molniya orbit when the relativistic oblateness perturbation is implemented. However, the standard orbit's angle of inclination, under the relativistic oblateness perturbation, exhibits an increasing secular variation. There has been nothing found in current literature to suggest a possible reason for this element's behavior.

\subsubsection{Discussion of Data Values}

Table 3.6 shows the minimums, maximums, means, and medians of the difference data for the orbital elements. This data that these values correspond to may be seen in graphical form in the figures presented previously in this section. The data in question was generated using the code outlined Chapter 2.

The true anomaly, $\theta$, is more effected for the standard orbit in both the standard and relativistic oblateness. However, the difference values are extremely close between each orbit case. This implies that there is very little difference between the 
Table 3.6: Summary of Data Values

\begin{tabular}{|c|c|c|c|c|c|}
\hline & & \multicolumn{2}{|c|}{ Standard Oblateness } & \multicolumn{2}{|c|}{ Relativistic Oblateness } \\
\hline & & $\begin{array}{l}\text { Standard } \\
\text { Orbit }\end{array}$ & $\begin{array}{l}\text { Molniya } \\
\text { Orbit }\end{array}$ & $\begin{array}{l}\text { Standard } \\
\text { Orbit }\end{array}$ & $\begin{array}{l}\text { Molniya } \\
\text { Orbit }\end{array}$ \\
\hline \multirow{4}{*}{$\begin{array}{c}\theta \\
\text { (radians) }\end{array}$} & Min & -3.2678 & -2.6793 & $-1.1757 \mathrm{E}-10$ & $-6.9146 \mathrm{E}-10$ \\
\hline & Max & $6 . \overline{1} \overline{9} \overline{7}^{--}$ & $6.0730^{-1}$ & $5 . \overline{3} \overline{2} \overline{\mathrm{E}}-4$ & $5.1826 \mathrm{E}-4$ \\
\hline & Mean & $-8.5404 \mathrm{E}-4$ & $2.3265 \mathrm{E}-4$ & $\overline{6} \overline{1} \overline{8} \overline{3} \mathrm{E}-\overline{5}$ & $2.2210 \mathrm{E}-5$ \\
\hline & Médian & -0.5445 & $-\overline{0} \cdot \overline{2} \overline{4} \overline{8} \overline{2}^{-1}$ & $\overline{3} . \overline{4} \overline{2} \overline{5}-\overline{\mathrm{E}}-\overline{5}$ & $7.7300 \mathrm{E}-6$ \\
\hline \multirow{4}{*}{$\begin{array}{c}a \\
(\mathrm{~km})\end{array}$} & Min & -9.7610 & -36.3296 & -0.0010 & -0.0021 \\
\hline & Max & $\overline{9} \overline{\overline{9}} \overline{8} \overline{9}^{-}$ & $\overline{5} \overline{1} . \overline{6} \overline{4} \overline{3}$ & $8 . \overline{4} \overline{9} \overline{9}-\bar{E}-6$ & $3.9202 \mathrm{E}-5$ \\
\hline & ${ }^{-} \bar{M}^{-}{ }^{-}$ & $\overline{7} . \overline{3} \overline{0} \overline{3} \overline{6}^{-}$ & $\overline{2} \overline{5} . \overline{8} \overline{4} \overline{5} \overline{6}^{-}$ & $-5 . \overline{1} 849 \mathrm{E}-4$ & ${ }^{-} \overline{0} . \overline{0} \overline{1} \overline{0}^{---}$ \\
\hline & ${ }^{-} \bar{M}$ èdian & $8.8 \overline{1} \overline{1} \overline{5}^{--}$ & $\overline{2} \overline{4} . \overline{5} \overline{9} \overline{0}^{-\cdots}$ & $-5.1853 \mathrm{E}-4$ & $-\overline{0} . \overline{0} \overline{1} \overline{0}^{---}$ \\
\hline \multirow{4}{*}{$\begin{array}{c}e \\
\text { (unitless) }\end{array}$} & Min & $-4.2974 \mathrm{E}-4$ & $-4.2159 \mathrm{E}-4$ & $-1.4417 \mathrm{E}-10$ & $-8.5419 \mathrm{E}-9$ \\
\hline & Max & $6.3 \overline{4} 7 \overline{6} \mathrm{E}-4$ & $5.1706 \mathrm{E}-4$ & $6.070 \overline{3} \mathrm{E}-8$ & $4 . \overline{6} 5 \overline{6} \overline{\mathrm{E}} \mathrm{\textrm {E }}-\overline{1} \overline{0}^{-}$ \\
\hline & Mean & $\overline{4} \overline{7} \overline{4} \overline{\mathrm{E}}-\overline{4}$ & $2.1391 \mathrm{E}-\overline{4}$ & $3.0 \overline{2} \overline{2}-\bar{E}-8$ & $-\overline{4} .0785 \mathrm{E}-\overline{9}^{-}$ \\
\hline & Médian & $5 . \overline{4} \overline{2} \overline{5}-\overline{-}-\overline{-}$ & $2.1133 \mathrm{E}-4$ & $\overline{3} . \overline{3} 0 \overline{3} \mathrm{E}-\overline{8}$ & $-4.0768 \mathrm{E}-\overline{9}^{-}$ \\
\hline \multirow{4}{*}{$\begin{array}{c}p \\
(\mathrm{~km})\end{array}$} & Min & -2.6022 & -2.8480 & -0.0016 & $-7.6181 \mathrm{E}-4$ \\
\hline & Max & $2 . \overline{2} \overline{8} \overline{5}$ & 8.5697 & $5.937 \overline{1} \mathrm{E}-6$ & $1.7735 \mathrm{E}-5$ \\
\hline & ${ }^{-} \bar{M}^{-}-\bar{n}^{-}$ & $\overline{0} . \overline{0} \overline{2} \overline{2} \overline{7}^{-}$ & 5.2019 & $-\overline{7} . \overline{9} \overline{2} 2 \mathrm{E}-\overline{4}$ & $-\overline{3} . \overline{4} \overline{2} \overline{9} \mathrm{E}-\overline{4}^{-}$ \\
\hline & Médiā' & $0 . \overline{0} \overline{8} \overline{6} \overline{6}^{-}$ & -7.7992 & $-7.7 \overline{9} 54 \mathrm{E}-4$ & $-\overline{3} . \overline{4} \overline{3} \overline{8} \mathrm{E}-\overline{4}^{-}$ \\
\hline \multirow{4}{*}{$\begin{array}{c}\Omega \\
\text { (radians) }\end{array}$} & Min & 0.0000 & -6.2831 & 0.0000 & -6.2832 \\
\hline & Max & $1 . \overline{4} \overline{4} \overline{3}-$ & $0.1810^{-}$ & $\overline{1} . \overline{3} 5 \overline{9} \overline{\mathrm{E}}-\overline{9}$ & $0.0000^{-}$ \\
\hline & Mean & $0 . \overline{7} \overline{2} \overline{1} \overline{1}^{-}$ & $-\overline{0} . \overline{0} \overline{3} \overline{1} \bar{c}$ & $6.7998 \mathrm{E}-10$ & $\overline{-0} \overline{1} \overline{2} \overline{2} \overline{2}^{-}$ \\
\hline & $\bar{M} \bar{e} \bar{d} \bar{a}-\bar{n}$ & $0.7 \overline{7} \overline{2} \overline{6}^{-}$ & 0.0882 & $6.8004 \mathrm{E}-10$ & 0.0000 \\
\hline \multirow{4}{*}{$\begin{array}{c}\omega \\
\text { (radians) }\end{array}$} & Min & -0.3134 & 0.0000 & $-5.2862 \mathrm{E}-7$ & -6.2832 \\
\hline & Max & $5 . \overline{9} \overline{6} \overline{8} \overline{8}^{-}$ & 6.2832 & $8.6658 \mathrm{E} 11$ & 6.2832 \\
\hline & Mean & $4 . \overline{2} \overline{6} 5 \overline{0}$ & $0.5282^{\cdots}$ & $-2.6 \overline{3} 94 \mathrm{E}-7$ & $7.2713 \mathrm{E}-\overline{4}$ \\
\hline & Médian & $4.7864^{--}$ & $2.6001 \mathrm{E}-4$ & $-2.6407 \mathrm{E}-7$ & $-6.1439 \mathrm{E}-8$ \\
\hline \multirow{4}{*}{$\begin{array}{c}i \\
\text { (radians) }\end{array}$} & Min & $-1.9468 \mathrm{E}-4$ & $-5.2546 \mathrm{E}-5$ & $-1.8407 \mathrm{E}-13$ & $-5.0848 \mathrm{E}-14$ \\
\hline & Max & $2.0 \overline{4} \overline{1} 0 \mathrm{E}-4$ & $1.5803 \mathrm{E}-4$ & $2.0363 \mathrm{E}-11$ & $1.58 \overline{9} \overline{\mathrm{E}}-1 \overline{3}$ \\
\hline & Mean & $\overline{3} . \overline{9} \overline{0} \overline{8}-\overline{6}$ & $9.5947 \mathrm{E}-5$ & $1.0153 \mathrm{E}-11$ & $\overline{1} . \overline{2} \overline{1} \overline{\mathrm{E}}-\overline{1} \overline{3}^{-}$ \\
\hline & Median & $4 . \overline{4} \overline{0} \overline{9}-\bar{E}$ & $8.8525 \mathrm{E}-5$ & $1.0156 \mathrm{E}-11$ & $1.2 \overline{6} \overline{6} \mathrm{E}-\overline{1} \overline{3}^{-}$ \\
\hline
\end{tabular}

different perturbation on this orbital parameter. The orders of magnitude for all of the comparison values (minimum, maximum, mean, and median) differ between half to one order of magnitude.

The semi-major axis is more effected for the Molniya orbit case under both perturbations. For the standard oblateness, the semi-major axis difference values are approximately one order of magnitude higher. This equates to a maximum instantaneous difference of approximately 51 kilometers. However, these difference values do not contribute to a secular change in this parameter as was mentioned previously. The difference values for the relativistic perturbation do contribute to the negative secular deviations pointed out in the last section.

The standard orbit's eccentricity, $e$, is more effected then the Molniya's eccentricity in both the standard and relativistic oblateness perturbations. The order of magnitudes of the comparison values are all similar in the case of the standard perturbation. However, in the case with the relativistic perturbation, there are order of magnitude differences. These differences are seen in all of the comparison values. 
In general, the order of magnitude differences are between one and two orders of magnitude lower for the Molniya orbit.

The semi-parameter, $p$, is more effected for the Molniya orbit under the standard oblateness perturbation; however, the standard orbit's semi-parameter is more effected under the influence of the relativistic oblateness perturbation. There are two orders of magnitude difference in the mean and median comparison values under the standard perturbation. In comparison, the standard orbit case, while under the influence of the relativistic perturbation, is approximately one order of magnitude higher. However, the order of magnitude analysis for the orbit cases under the relativistic perturbations is inconclusive. Therefore, the same argument could be made for the Molniya orbit case. Further analysis, over a greater propagation time would be needed to fully classify this difference.

The RAAN, $\Omega$, shows a greater difference for the Molniya orbit under either perturbation. For the standard oblateness perturbation there is a one order of magnitude difference for all comparison values. There are up to nine orders of magnitude difference on the comparison values. However, extrapolated on the trends discussed in the previous section, the maximum and the median comparison values are really indeterminate.

The argument of parigee, $\omega$, is more effected in the standard case orbit while either perturbations are being applied. Under the standard oblateness perturbation, the median comparison value of the Molniya orbit is one order of magnitude lower. There are three orders of magnitude difference between the mean comparison values. However, taking the trend analysis from the previous section into consideration, these results are really indeterminate as well.

The inclination, $i$, is an interesting parameter because the Molniya orbit is more effected under the standard perturbation. This is what should be expected since the inclination of the Molniya orbits is considerably greater then the standard orbit. However, the standard orbit's inclination is more effected under the relativistic perturbation. Under the standard perturbation, there is a one order of magnitude difference in all comparison values. Under the relativistic oblateness perturbation, there is a one order of magnitude difference in the minimum comparison value and a two order of magnitude difference in all other comparison values.

\subsection{Discussion of Specific Force Data}

The specific force components $(\widehat{I}, \widehat{J}$, and $\widehat{K})$, as well as the magnitude that manifests from the relativistic oblateness perturbation are shown in Figure 3.9. These quantities were calculated through a post processing routine that used the outputted state vectors from the integration schemes described in Chapter 2. The post processing 
routine was a slightly modified version of the code found in Appendix J.

\subsubsection{Relativistic Oblateness Perturbation}

\subsubsection{Standard Orbit Case}
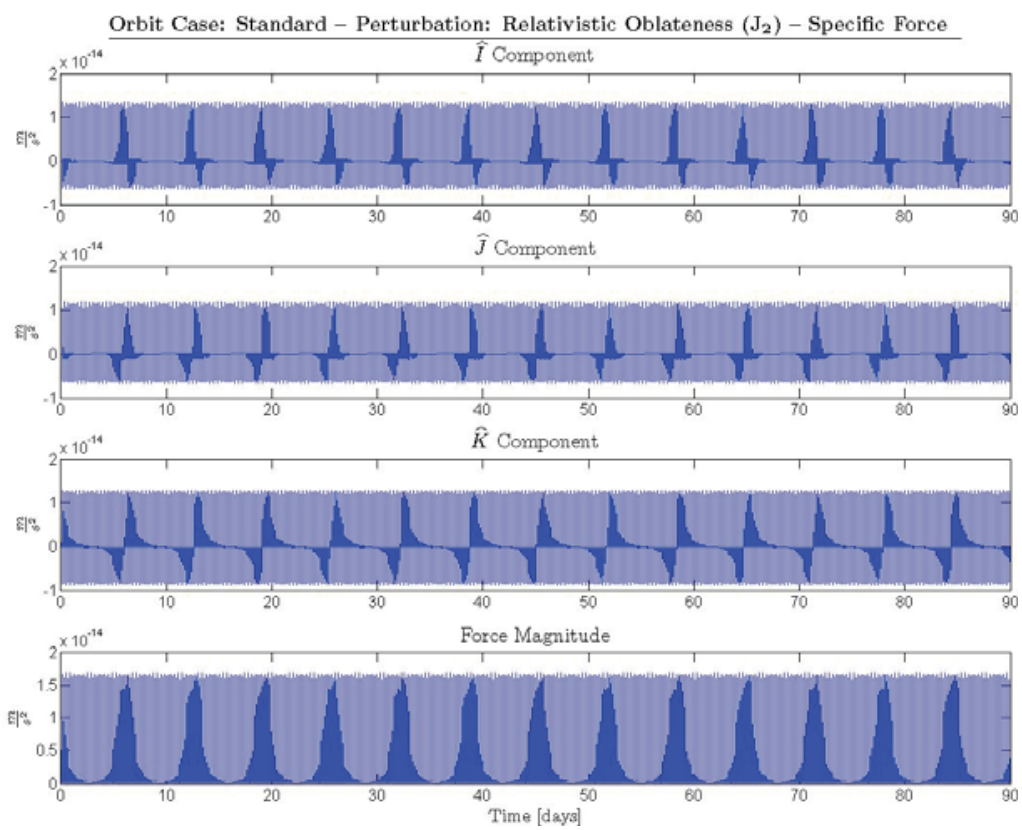

Figure 3.9: Standard Orbit Specific Force for Relativistic Oblateness versus Time

All of the components and the magnitude of the specific force plots shown in Figure 3.9 exhibit short term periodicity. All of the components also show full reversal. It is possible to see that each of the components exhibit short period modulation by inspection of of Figure 3.9. The mean value of the components also remains relatively constant. The magnitude, shown in the bottom plot of Figure 3.9, exhibits short period modulation. However, the specific force magnitude maintains a relatively constant mean value. This is based on the plot density.

\subsubsection{Molyina Orbit Case}

Figure 3.10 shows the components and magnitude of the specific force induced by the relativistic oblateness perturbation on the Molniya orbit. By inspection of the plots contained in Figure 3.10, it is possible to see that all of the components exhibit short term periodic modulation. The combined effect of the components is shown by the 

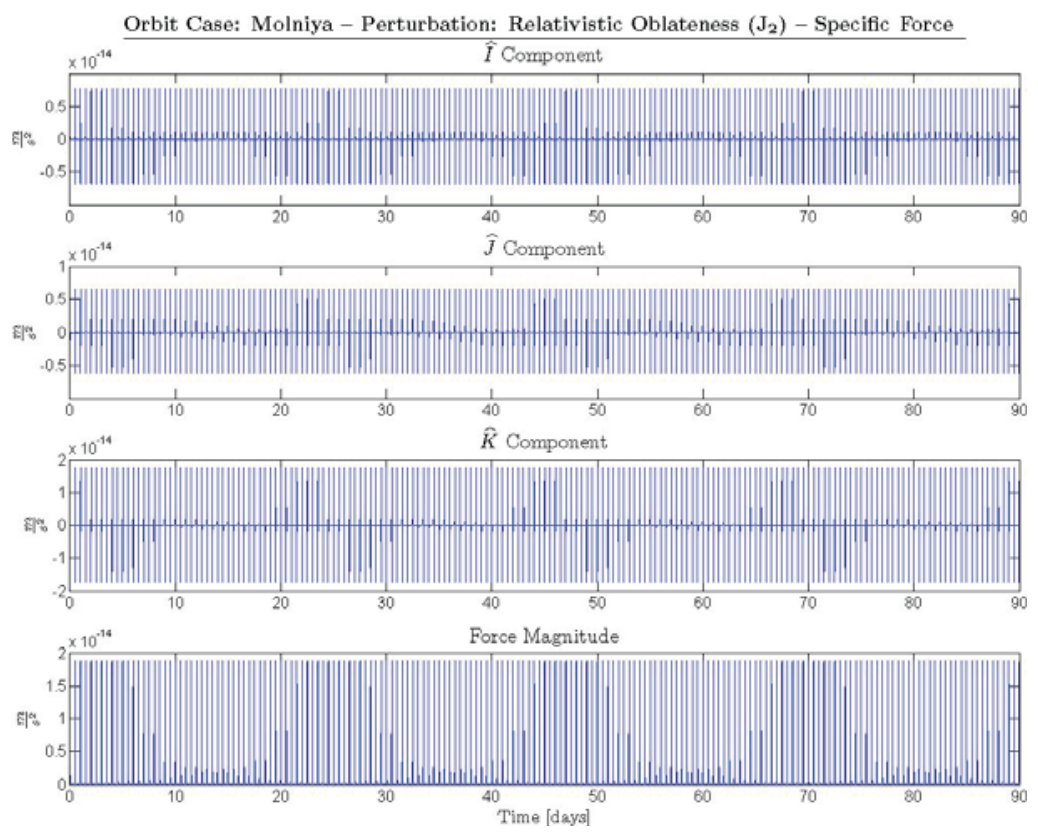

Figure 3.10: Molniya Orbit Specific Force for Relativistic Oblateness versus Time

force magnitude. The bottom plot of Figure 3.10 does show that the specific force magnitude does hold a relatively constant mean value.

\subsubsection{Standard Oblateness Perturbation}

\subsubsection{Standard Orbit Case}

The specific force induced by standard oblateness perturbation on the standard orbit is seen in Figure 3.11. In this case, the specific force components exhibit both short and long term periodic modulation. This same behavior is exhibited, although to a lesser degree, in the specific force magnitude. The long term periodic modulation implies that the specific force has a moving mean value. However, the long term periodic variation in the magnitude is of little consequence because the mean magnitude remains fairly constant.

\subsubsection{Molyina Orbit Case}

Figure 3.12 shows the specific force induced by the standard oblateness perturbation when applied to the Molniya orbit. Upon inspection, it is possible to see that all components of this force exhibit only short term periodic fluctuation. This is also the 
same variation seen in the force magnitude. From the force magnitude plot (bottom plot in Figure 3.12) it is possible to infer that the magnitude of this force does remain fairly constant. 

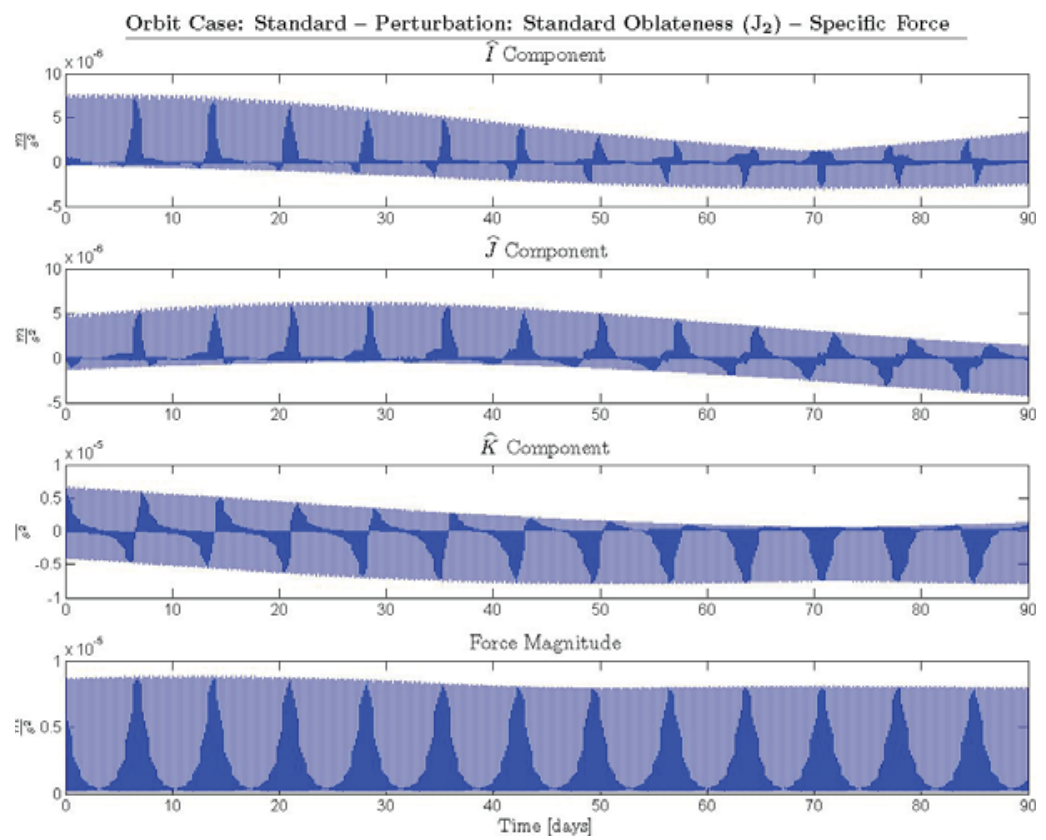

Figure 3.11: Standard Orbit Specific Force for Standard Oblateness versus Time
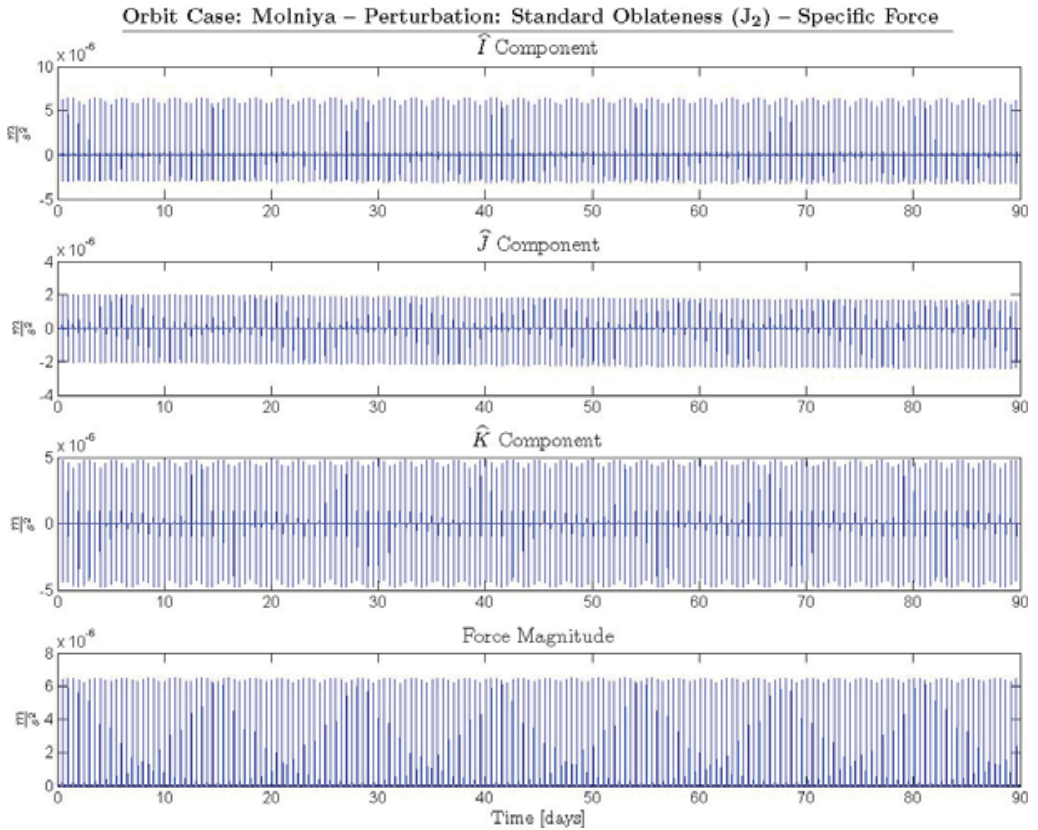

Figure 3.12: Molniya Orbit Specific Force for Standard Oblateness versus Time 


\subsubsection{Comparison of Specific Force Data}

Table 3 shows the specific forces that are generated under the different perturbations for the two different test case orbits. The component values shown in Table 3.7 are expressed in the geocentric coordinates where the integrations were carried out. The units of the given values are Newtons.

Table 3.7: Summary of Force Data Values in Newtons

\begin{tabular}{|c|c|c|c|c|c|}
\hline & & \multicolumn{2}{|c|}{ Standard Oblateness } & \multicolumn{2}{|c|}{ Relativistic Oblateness } \\
\hline & & $\begin{array}{l}\text { Standard } \\
\text { Orbit }\end{array}$ & $\begin{array}{c}\text { Molniya } \\
\text { Orbit }\end{array}$ & $\begin{array}{l}\text { Standard } \\
\text { Orbit }\end{array}$ & $\begin{array}{c}\text { Molniya } \\
\text { Orbit }\end{array}$ \\
\hline \multirow{4}{*}{$\begin{array}{c}x \\
\text { Component }\end{array}$} & Min & $-3.0423 \mathrm{E}-6$ & $-3.2203 \mathrm{E}-6$ & -6.3920 E -15 & -6.7929 E -15 \\
\hline & $\operatorname{Max}$ & $\overline{7} . \overline{6} 5 \overline{2} \overline{\mathrm{E}}-\overline{6}$ & $6.4808 \mathrm{E}-6$ & $1.3470 \mathrm{E}-14^{-}$ & $7 . \overline{2} \overline{7} \overline{\mathrm{E}}-\overline{1} \overline{5}^{-}$ \\
\hline & Mean & $1 . \overline{2} 8 \overline{6} \overline{\mathrm{E}}-7$ & $-3.9026 \mathrm{E}-11$ & $2.9774 \mathrm{E}-16$ & $-2.2701 \mathrm{E}-17$ \\
\hline & Médian & $-3.2454 \mathrm{E}-8$ & $-5.06 \overline{18} \mathrm{E}-\overline{9}^{-}$ & $-6.9152 \mathrm{E}-17^{-}$ & $-1.5839 \mathrm{E}-18$ \\
\hline \multirow{4}{*}{$\begin{array}{c}y \\
\text { Component }\end{array}$} & Min & $-2.2845 \mathrm{E}-6$ & $-2.4222 \mathrm{E}-6$ & $-6.5751 \mathrm{E}-15$ & $-6.1795 \mathrm{E}-15$ \\
\hline & Max & $6 . \overline{2} 5 \overline{4} 0 \mathrm{E}-6$ & $2.0221 \mathrm{E}-6$ & $1.1796 \mathrm{E}-14$ & $6.3781 \mathrm{E}-15^{-}$ \\
\hline & Mean & $1.5 \overline{4} 0 \overline{0} \mathrm{E}-\overline{7}$ & $-4.3278 \mathrm{E}-10$ & $9.3342 \mathrm{E}-17$ & $2.460 \overline{1} \mathrm{E}-1 \overline{9}$ \\
\hline & Médian & $-1.8304 \mathrm{E}-8$ & $-4.4576 \mathrm{E}-10$ & $-6.4501 \mathrm{E}-17^{-}$ & $2.87 \overline{4} 0 \mathrm{E}-\overline{2} \overline{1}^{-}$ \\
\hline \multirow{4}{*}{$\begin{array}{c}z \\
\text { Component }\end{array}$} & Min & $-7.9387 \mathrm{E}-6$ & $-4.7849 \mathrm{E}-6$ & -8.7283 E -15 & $-1.7159 \mathrm{E}-14$ \\
\hline & $\operatorname{Max}$ & $6.6 \overline{6} \overline{8} \bar{E}-\overline{6}$ & $4.7861 \mathrm{E}-6$ & $1.2881 \mathrm{E}-14$ & $\overline{1} . \overline{4} \overline{6} \overline{1} \mathrm{E}-1 \overline{4}$ \\
\hline & Mean & $-3.9613 \mathrm{E}-7$ & $1.546 \overline{1} \mathrm{E}-10$ & $2.5341 \mathrm{E}-16^{-}$ & $6.7 \overline{4} \overline{\mathrm{E}}-1 \overline{9}$ \\
\hline & Médian & $1.63 \overline{8} \bar{E}-7$ & $6.2050 \mathrm{E}-12$ & $-4.7312 \mathrm{E}-17^{-}$ & $1.8596 \mathrm{E}-20^{-}$ \\
\hline \multirow{4}{*}{ Magnitude } & Min & $1.6380 \mathrm{E}-7$ & $6.2615 \mathrm{E}-9$ & $1.0676 \mathrm{E}-16$ & $1.6441 \mathrm{E}-18$ \\
\hline & Max & $8.8 \overline{8} \overline{4} \overline{1}-6$ & $6.4826 \mathrm{E}-6$ & $1.6818 \mathrm{E}-14$ & $1.87 \overline{7} \mathrm{E}-14$ \\
\hline & Mean & $1 . \overline{4} \overline{3} \overline{\mathrm{E}}-\overline{6}$ & $3.9380 \mathrm{E}-7$ & $2.2605 \mathrm{E}-15$ & $8.5347 \mathrm{E}-16$ \\
\hline & Mèdian & $3.8642 \mathrm{E}-7$ & $1.2752 \mathrm{E}-8$ & $2.6582 \mathrm{E}-16$ & $3 . \overline{6} \overline{6} \overline{3} \mathrm{E}-1 \overline{8}$ \\
\hline
\end{tabular}

Under the influence of the standard oblateness perturbation the Molniya orbit is less effected. Their maximum order of magnitude differences in the specific force components is two. However, this trend is also echoed in the magnitude values of specific force.

The standard case orbit is also more effected when subjected to the relativistic oblateness perturbation. Three is maximum order of magnitude between specific force values while the different orbit cases are under the influence of the relativistic perturbation. Therefore it is not surprising that the same conclusion may also be drawn from the magnitude data comparison values.

Using the data contained in Table 3.7 it is also possible to draw the conclusion that the relativistic oblateness perturbations are considerably weaker then the standard oblateness perturbation. This should not come as a surprise because of the data that has been present thus far. As can be seen in Table 3.7, there are up to thirteen orders of magnitude difference between the specific forces generated by the standard and relativistic perturbation. 


\section{Chapter 4}

\section{Conclusions}

\subsection{Proof of Concept}

As was stated in the introduction, a major goal of this work was to develop a proof of concept for the hypothesis that new, and more optimal, solutions for trajectory optimization problems could be found if different gravitational frameworks were considered. In order for this to be a viable possibility, a difference would have to be found between the relativistic and non-relativistic formulations. It is known that planetary trajectories differ depending on whether they are calculated in relativistic or non-relativistic framework [10]. This leads to the more interesting question of, "If the orbits are known to be different depending on the formalism that is being applied during the calculation of the trajectory, does this difference manifest in some quantifiable form in other aspects of orbital theory when comparing different frameworks?" If the answer to this question were "no," then there would be no difference in the relativistic versions of the perturbations presented in this paper, and there would be no point in continuing this work. However, as the results show in the last section, the answer to this question is indeed "yes."

The model that was derived and utilized in this research is essentially a modified form of the model that is used in classical Newtonian orbit theory. The relativistic perturbation terms that were used in the models, while being derived in a fully relativistic format [29], were implemented in the same manner as a standard perturbing term for the methods. This implementation was possible because the form of the relativistic perturbations could be manipulated into a form that allowed the relativistic forces to be treated as a standard perturbation term [6].

The difference in orbital parameter deviation, specific force components, and specific force magnitudes were easily seen in the oblateness perturbations presented in the last section because the same effect was looked at, and derived, in both non- 
relativistic and relativistic frameworks. The differences that were exhibited between the effects produced by the non-relativistic perturbing terms and the relativistic perturbing terms are significant, albeit the relativistic effects are comparatively smaller then the non-relativistic terms.

However, as the speed and distance capabilities of spacecrafts increase, the deviations produced by the relativistic perturbing terms will also grow proportionally [29]. The differences between the effects produced by the non-relativistic and relativistic perturbing terms should be taken as unequivocal evidence that it would be possible to leverage the relativistic framework to develop new orbit optimization tools.

\subsection{Relevance of Presented Work}

The possibilities for application of the work that has been presented in this thesis is limited to a subset of orbit maintenance problems at this point in time $[6,14$, 28]. These orbit maintenance problems deal with timing issues that are seen with geosynchronous satellites. However, the current state of technology was not the main focus when this research was begun.

The idea that the human race could reach beyond our solar system to others, or even another galaxy is quickly becoming a reality based on some of the work that is being done on advanced propulsion systems which was presented in Chapter 1. The fact that is seldom realized is that it is necessary to develop the hardware for cutting edge technology in tandem with the theory that must be applied to utilize the new technology to its fullest potential. However, it would seem even more beneficial for the hardware to lag behind the theory in space applications. This is what was seen in the early days of space exploration as was pointed out in 1.

The work that has been presented within this thesis is a small step in the engineering community's ability to optimize trajectories that will take us beyond our solar system, but it is a necessary one. This work serves as a bridge between methods, techniques, and theories that until recently have been dealt with completely within the domains of theoretical and mathematical physics. The present work also provides the incontrovertible evidence that spacecraft traveling at relatively low velocities for short distances will experience a small contribution of relativistic perturbing forces as pointed out in the previous chapter. However, as distance and speed capabilities of spacecrafts increase with the constant advancements and scientific contributions, the

slight trajectory deviations seen at the low energy situations used in the simulations presented here will increase greatly. 


\subsection{Implications and Future Work}

The work presented within this thesis has been conducted using a modified form of the Newtonian equations of motion. The power of relativistic optimization techniques would benefit greatly from formulating the trajectory optimization problem beginning with first principles in the GTR. A reformulation of the trajectory optimization problem within the GTR would allow for the more complete use of the differential geometric methods that are commonly used in this field. This is an added benefit for optimization work is that there is an entire theory of geometric optimization and control methods that could be reworked and applied to the problem at hand.

The theories of geometric optimization and control are not new ideas to the mathematics community. However, they have not found many applications within the realm of engineering with the exception of a limited range of problems. The main reason for this is because there has never been a necessity to do so. However, considering that geometric methods are the language of the GTR, and if a reformulation of the trajectory optimization problem could be obtained, it would seem to be a mistake to neglect the possibility of leveraging these tools. These tools could afford the possibility of having currently unknown solutions manifest from the equations for both relativistic and non-relativistic trajectories.

However, these techniques may not be limited to the area of trajectory optimization. Geometric optimization and control techniques are powerful in their own right. It may be possible for the application of these techniques in other areas of engineering once their usefulness has been demonstrated in an applied branch of mechanics. 
Appendices 



\section{Appendix A}

\section{Abbreviations}

GTR General Theory of Relativity

MoI Moment of Inertia

EoM(s) Equation(s) of Motion

PDE Partial Differential Equations

COE Classical Orbital Elements 


\section{Appendix B}

\section{Symbols}

Please note that $\boldsymbol{\Delta}$ has NO meaning of its own. It is used as a place holder for explanation of the symbols only; therefore, any appropriate variable, or number where applicable, may take its place.

\section{Superscripts and Overbars}

ム* Specific Quantity (Mass component removed, e.g. specific force)

$\overrightarrow{\boldsymbol{\Delta}}$ A vector

$\hat{\boldsymbol{\Delta}}$ A unit vector

\section{Subscripts}

$\boldsymbol{\Delta}_{\oplus}$ Earth Related Quantity

$\boldsymbol{\Delta}_{r}$ A radius

$\boldsymbol{\Delta}_{\mathrm{g}}$ Gravitational quantity

$\boldsymbol{\Delta}_{\text {rel }}$ Relativistic formulation of a normally non-relativistic quantity

ム. Arbitrary celestial body quantity

$\boldsymbol{\Delta}_{\text {pert }}$ Regularly used quantity that is associated with a perturbing term

$\boldsymbol{\Delta}_{\text {ST }}$ Spherical term

$\boldsymbol{\Delta}_{\text {GP }}$ Geodesic Precession term 
$\boldsymbol{\Delta}_{\mathbf{L T}}$ Lense-Thirring term

$\boldsymbol{\Delta}_{\text {RJ2 }}$ Relativistic Oblateness term

$\boldsymbol{\Delta}_{\mathbf{R E}}$ Rotational Energy term

\section{Units}

$m$ Meters

kg Kilograms

\section{Normal Fonts}

a Semimajor axis

$b$ Semiminor axis

$J$ Polar Moment of Inertia

$I_{i j}$ Moment of Inertia Tensor, or Inertia Tensor Component

$M$ Mean anomaly

E Eccentric anomaly

$\theta$ True anomaly

$T$ Time at periapsis of an orbit

$G$ Standard gravitational constant

$\mu$ Standard gravitational parameter

$\mathcal{P}$ Keplerian period

$\mathbf{J}_{\mathbf{\Delta}}$ Oblateness factor

$\vec{\Omega}$ Spin vector 


\section{Appendix C}

\section{Relevant Properties of Earth}

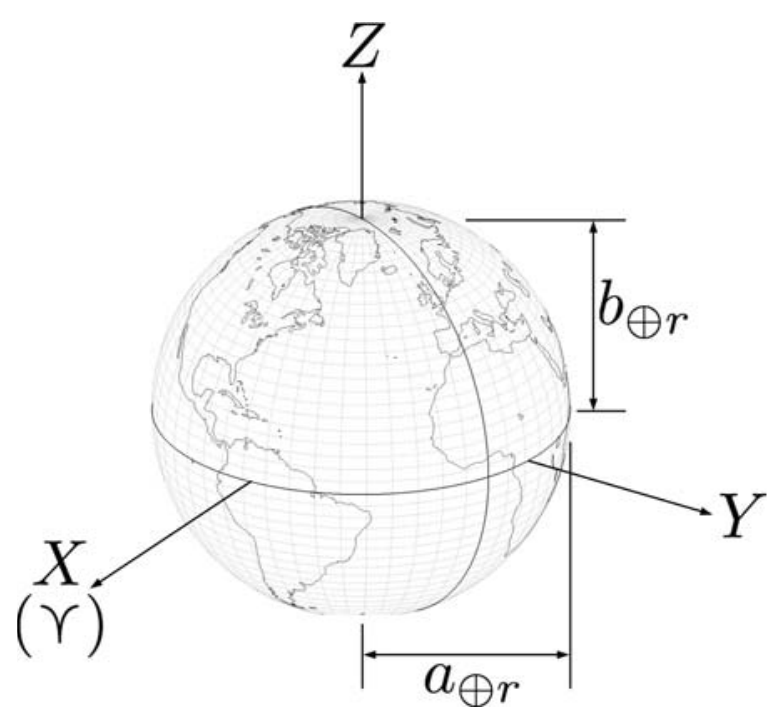

Figure C.1: Earth Geometry and Coordinate System 
Table C.1: Earth Parameters [31]

\begin{tabular}{|c|c|c|c|}
\hline Parameter & Symbol & Value & Units \\
\hline \hline Equatorial Radius & $a_{\oplus r}$ & 6378.136 & $\mathrm{~m}$ \\
\hline Polar Radius & $b_{\oplus r}$ & 6354.751 & $\mathrm{~m}$ \\
\hline Mass & $M_{\oplus}$ & $5.9723 \mathrm{E} 24$ & $\mathrm{~kg}$ \\
\hline Polar MoI & $C_{\oplus}, J_{\oplus}$, or $I_{\oplus Z Z}$ & $8.0359 \mathrm{E} 37$ & $\mathrm{~kg} \cdot \mathrm{m}^{2}$ \\
\hline Equatorial MoI & $A_{\oplus}$, or $I_{\oplus X X}$ and $I_{\oplus Y Y}$ & $8.0096 \mathrm{E} 37$ & $\mathrm{~kg} \cdot \mathrm{m}^{2}$ \\
\hline Specific Polar MoI & $C_{\oplus}^{*}, J_{\oplus}^{*}$, or $I_{\oplus Z Z}^{*}$ & $1.3455 \mathrm{E} 13$ & $\mathrm{~m}^{2}$ \\
\hline Specific Equatorial MoI & $A_{\oplus}^{*}$ or $I_{\oplus X X}^{*}$ and $I_{\oplus Y Y}^{*}$ & $1.3411 \mathrm{E} 13$ & $\mathrm{~m}^{2}$ \\
\hline
\end{tabular}




\section{Appendix D}

\section{Theoretical Propagation Code}

This code was written for and implemented in Matlab. Any questions about its implementation or use may be sent to the email in the comments section.

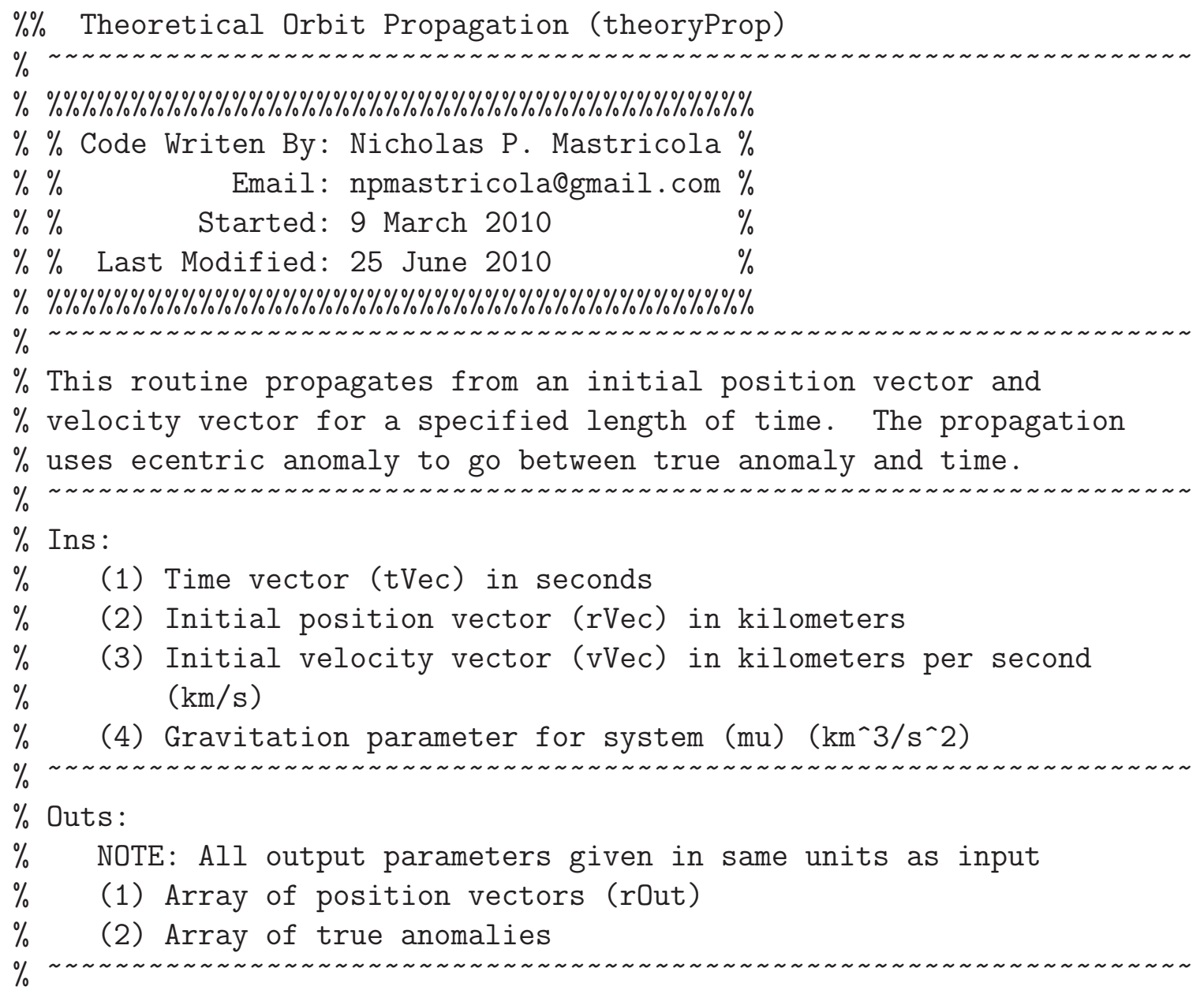


$\%$ Optional Outs:

$\% \quad$ NOTE: All output parameters given in same units as input

$\%$

$\%$ (1) Regular time (tOut): This is the same time vector that was

$\% \quad$ input. It has been included as a convenience during plotting

$\% \quad$ routines.

$\%$

$\% \quad$ (2) Orbit time vector (tCalc): Input time vecter shifted such

$\% \quad$ that $t(0)=0$ at periapsis.

$\%$

$\% \quad$ (3) Time shift (tShift): Single value used to shift the input

$\%$ time vector.

\%

$\%$ Calls:

$\%$ (1) [rOut thOut tOut] = theoryProp ( $t V e c, r V e c, v V e c, m u)$

$\%$ (2) [rOut thOut tout tCalc] = theoryProp ( $t V e c, r V e c, v V e c, m u)$

$\%$ (3) $[r$ rout thOut tout tCalc tShift $]=$

$\% \quad$ theoryProp (tVec, rVec, vVec, mu)

$\%$

$\% \quad$ NOTE: If units are included, then they must be paired with the

$\% \quad$ correct parameter.

$\%$

$\% \quad$ (4) $\ldots$ = theoryProp (tVec, 'tUnits', rVec, 'rUnits', $\ldots$

$\% \quad$ vVec, 'vUnits', mu, 'muUnits')

$\%$

$\%$ main function

function [rOut thOut tOut tCalc tShift] = theoryProp(varargin)

$\%$ input handling

switch nargin

case $\{1,2,3\}$

error('Insufficient input.')

case 4

$\mathrm{tVec}=\operatorname{varargin}\{1\}$

$\mathrm{rVec}=\operatorname{varargin}\{2\}$

$\mathrm{vVec}=\operatorname{varargin}\{3\} ;$

$\mathrm{mu}=\operatorname{varargin}\{4\}$;

otherwise

end

error('Unexpected inputs.')

$\%$ getting CoEs from rVec and vVec 
[th e a p iAng omega] = RV2COE (rVec, vVec,mu, 'outputForm', ...

'array', 'caseWarning', 'off');

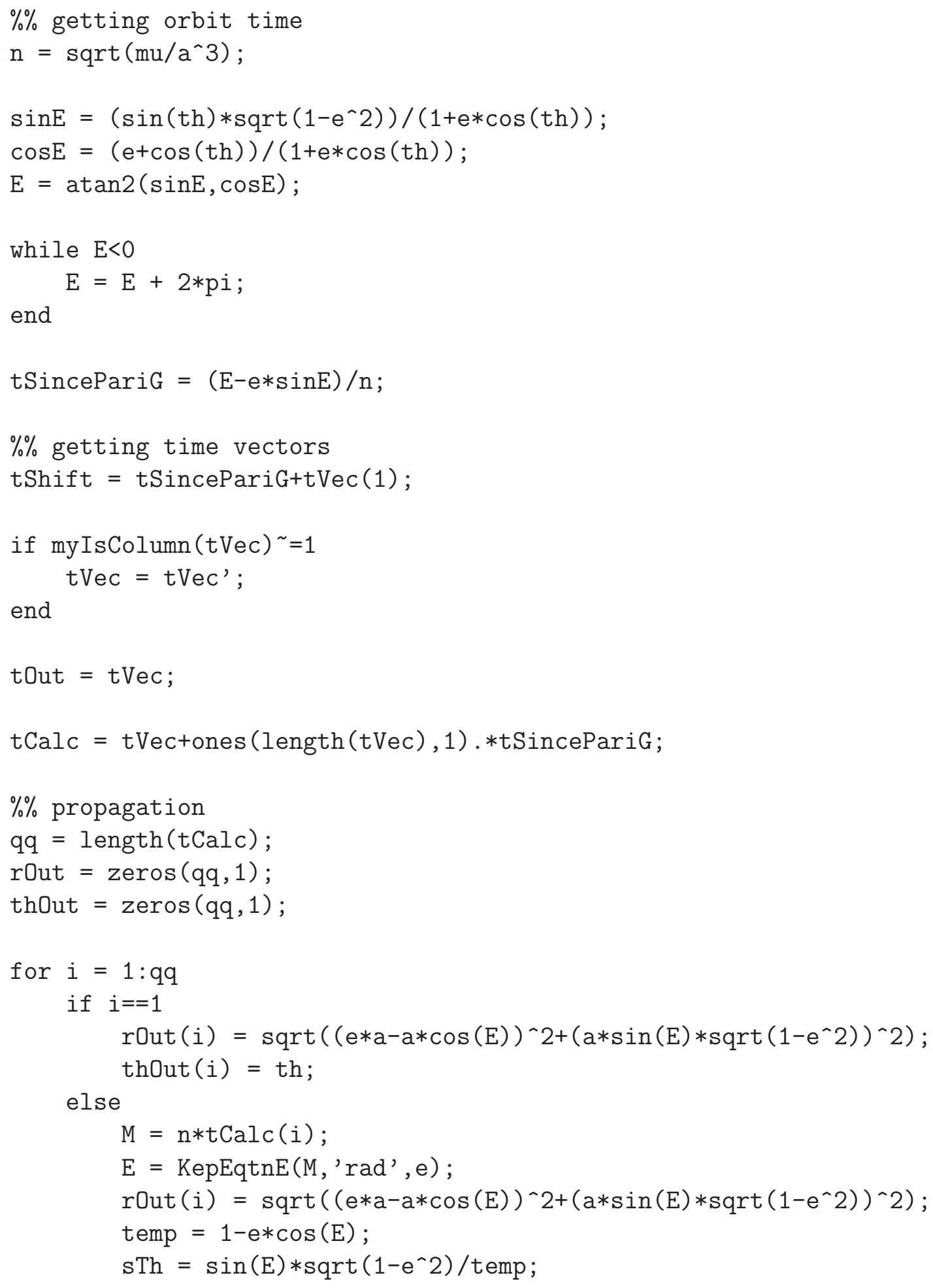




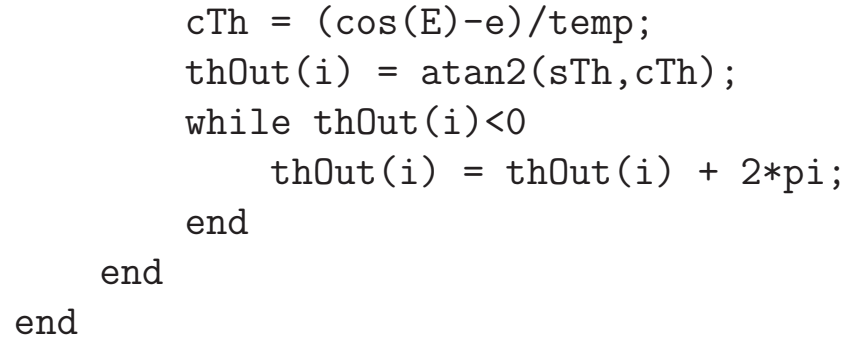




\section{Appendix E}

\section{Unperturbed State Space Code}

This code is the unmodified planet-satellite system. It will be expanded on by addition of later perturbing terms.

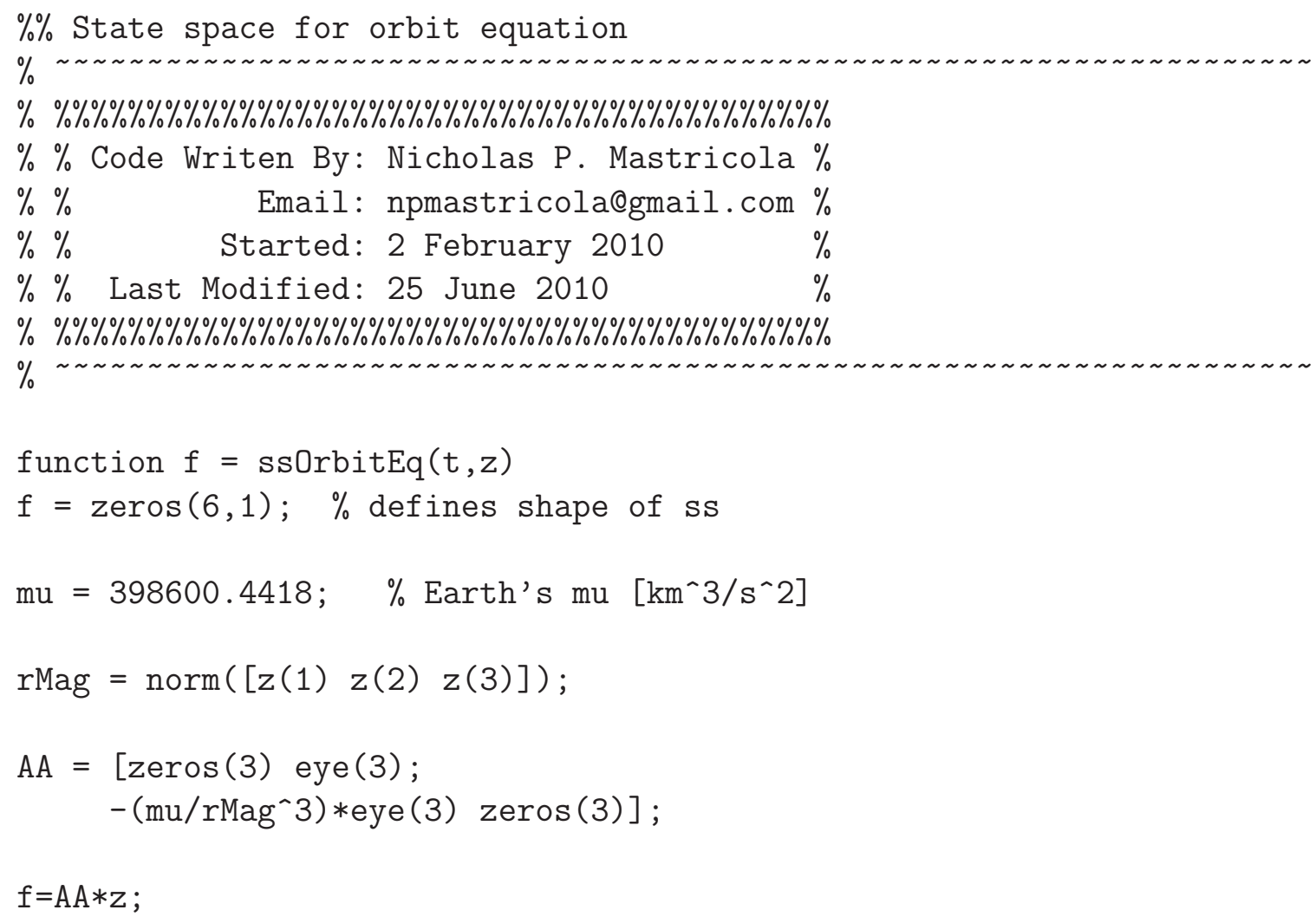




\section{Appendix F}

\section{Modified State Space for Non-Relativistic Perturbing Terms}

This code was used as the state space for non-relativistic perturbing terms.

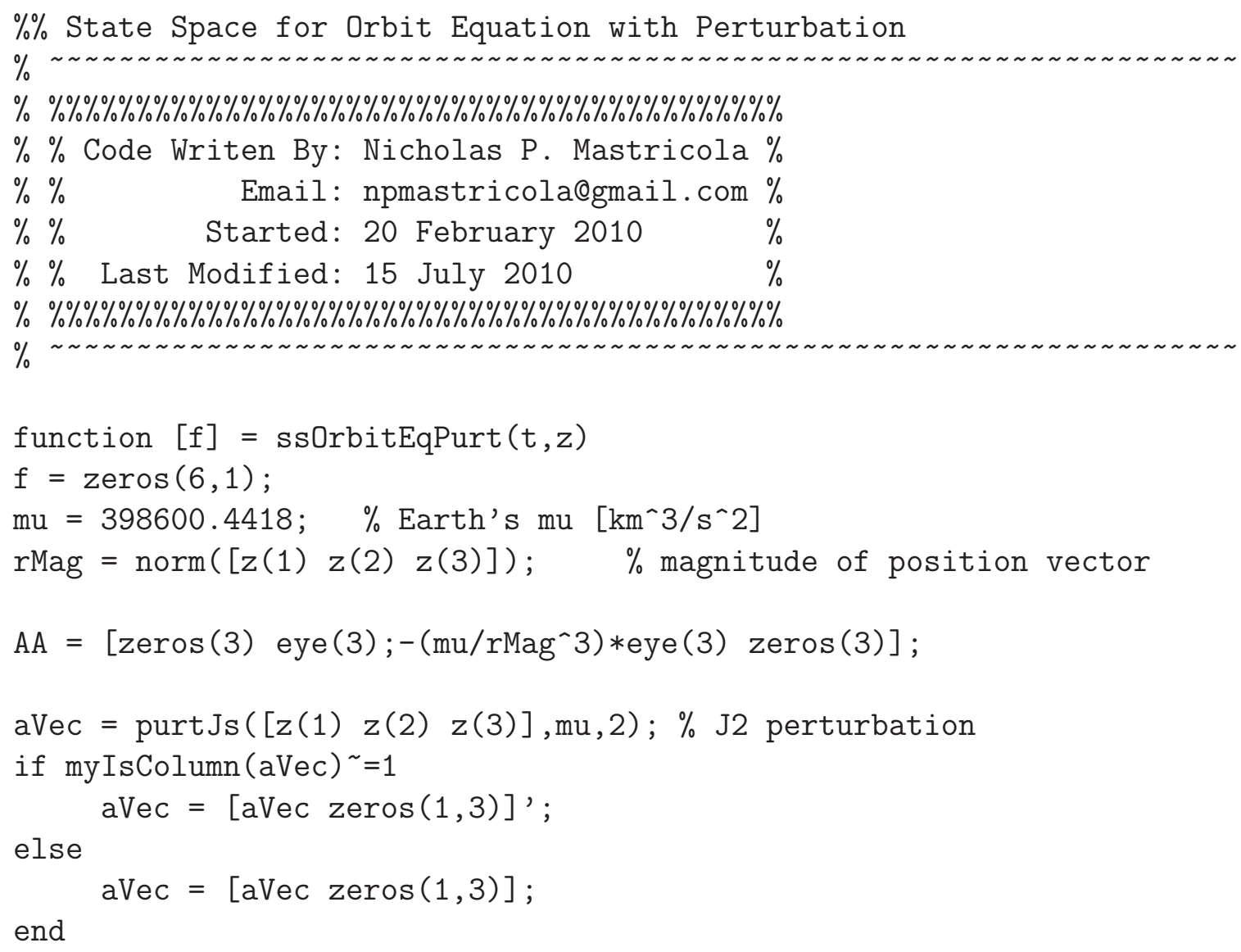




$$
\begin{aligned}
& \text { MM }=[\operatorname{zeros}(3) \operatorname{zeros}(3) ; \operatorname{eye}(3) \operatorname{zeros}(3)] ; \\
& f=A A * z+M M * a V e c ;
\end{aligned}
$$




\section{Appendix G}

\section{Modified State Space for Relativistic Perturbing Terms}

This code was used as the state space for relativistic perturbing terms. It should be noted that this code has been written to facilitate a number of different relativistic perturbations. Therefore, there are some features that are not used for every term (i.e. workbar() function and global and persistent variables).

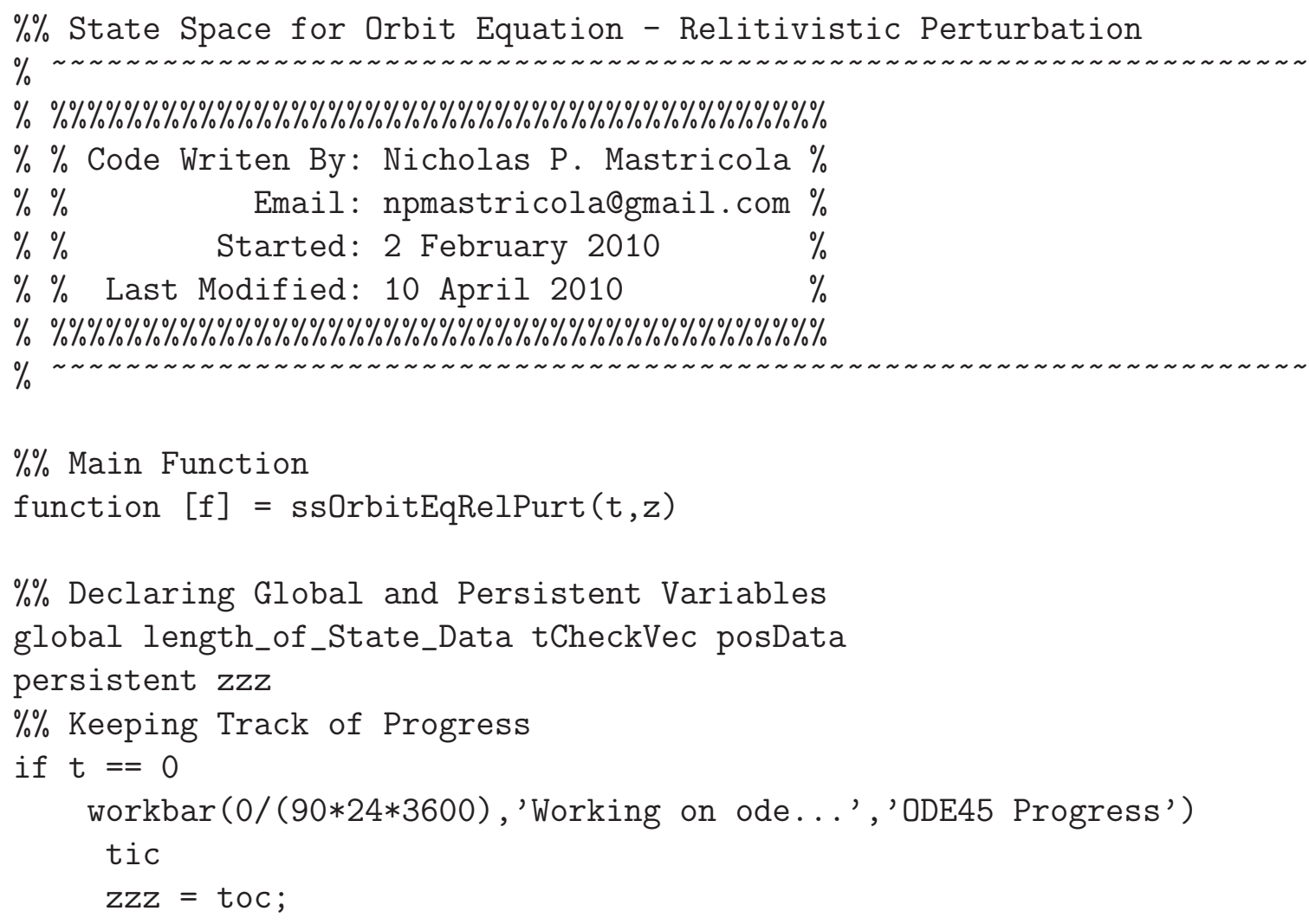




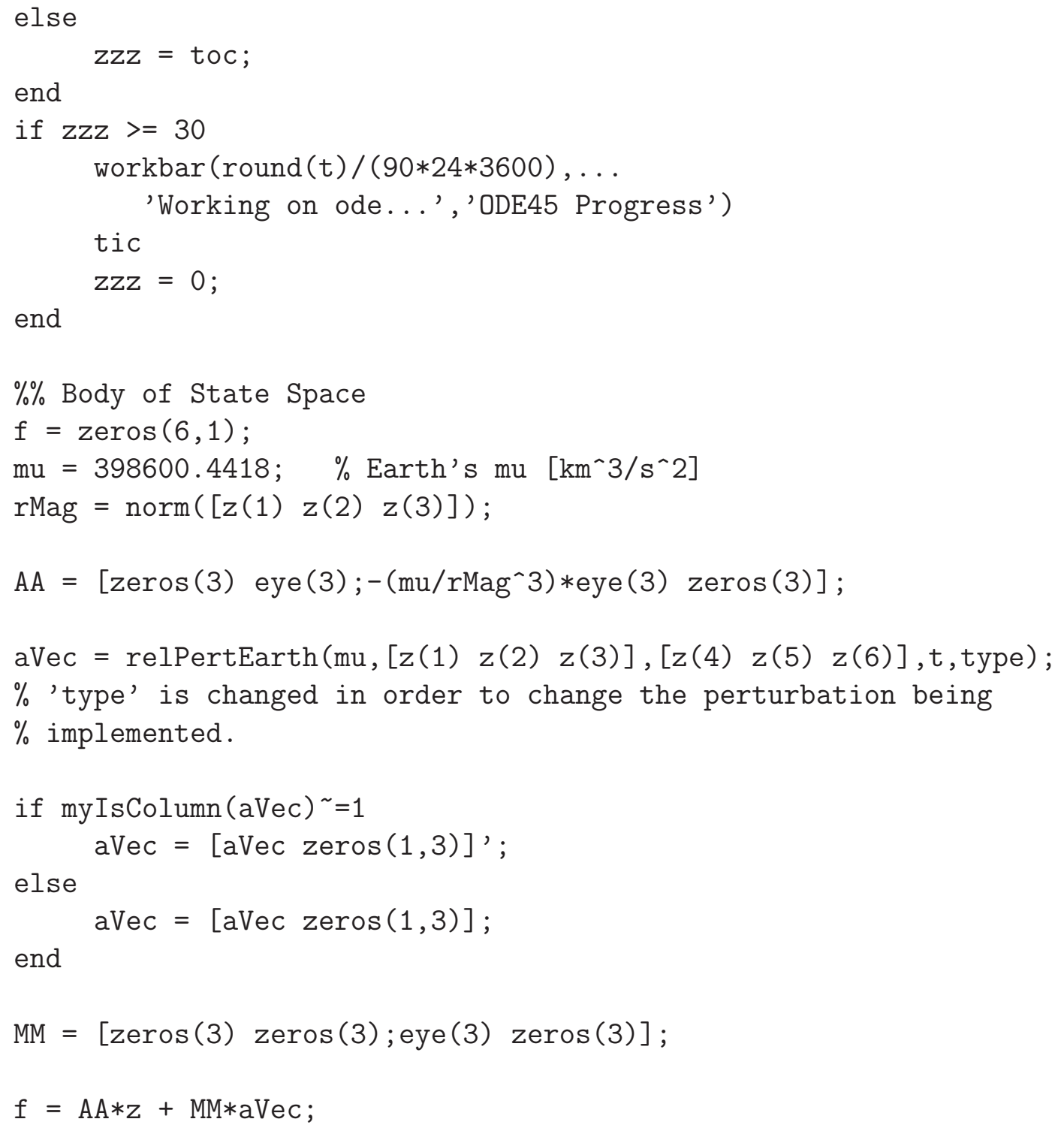




\section{Appendix $\mathbf{H}$}

\section{Commonly Used Equations}

This list has been included here because some "constants" (which the author will not refer to as semi-constants) in relativity do vary with position and velocity of the test particle in question. Equations that are referenced in the main body of this thesis that are not explicitly stated are also defined, or explained, here.

\section{Semi-constants}

$$
\begin{gathered}
\beta \equiv \frac{v}{c} \\
\gamma \equiv \frac{1}{\sqrt{1-\beta^{2}}}=\frac{1}{\sqrt{1-\left(\frac{v}{c}\right)^{2}}}=\frac{c}{\sqrt{c^{2}-v^{2}}}
\end{gathered}
$$




\section{Appendix I}

\section{The Twins Problem Solution}

Using the Minkowskian metric, proper time takes the form [16]

$$
\begin{aligned}
d \tau & =\sqrt{\eta_{\mu \nu} d x^{\mu} d x^{\nu}} \\
& =\sqrt{d t^{2}-\frac{1}{c^{2}}\left(d x^{2}+d y^{2}+d z^{2}\right)} .
\end{aligned}
$$

To find the relationship between proper time and coordinate time, the right hand side of Equation I.2 needs to be multiplied by $\frac{d t}{d t}$. Thus,

$$
\begin{aligned}
d \tau & =\sqrt{d t^{2}-\frac{1}{c^{2}}\left(d x^{2}+d y^{2}+d z^{2}\right)}\left(\frac{d t}{d t}\right) \\
& =\sqrt{d t^{2}-\frac{1}{c^{2}} d r^{2}}\left(\frac{d t}{d t}\right) \\
& =\sqrt{\frac{d t^{2}}{d t^{2}}-\frac{1}{c^{2}} \frac{d r^{2}}{d t^{2}}} d t .
\end{aligned}
$$

To continue this manipulation, it is necessary to realize that $\frac{d r^{2}}{d t^{2}}$ is the normal 3-space velocity of the particle in motion. Hence,

$$
d \tau=\sqrt{1-\frac{v^{2}}{c^{2}}} d t .
$$

Tradition dictates that this quantity is normally expressed as

$$
\frac{d t}{d \tau}=\frac{1}{\sqrt{1-\beta^{2}}}=\gamma \quad \text { where } \quad \beta=\frac{v}{c} .
$$

Equation I.5 is used to compute the amount of proper time that passes. If the 
spacecraft is traveling at $0.8 c$ and the half-length of the spacecraft journey is forty years, the calculations are:

$$
\begin{aligned}
d \tau & =\int_{t_{0}}^{t_{1}} \frac{1}{\gamma} d t \\
& =\int_{0}^{80} \sqrt{1-\frac{0.8^{2} c^{2}}{c^{2}}} d t \\
& =\int_{0}^{80} \sqrt{1-0.8^{2}} d t \\
& =80 \sqrt{0.36} \\
& =48 \text { years }
\end{aligned}
$$

Thus, the time that the twin in the spacecraft would experience passes is 48 years. 


\section{Appendix $\mathbf{J}$}

\section{Relativistic Perturbation Code}

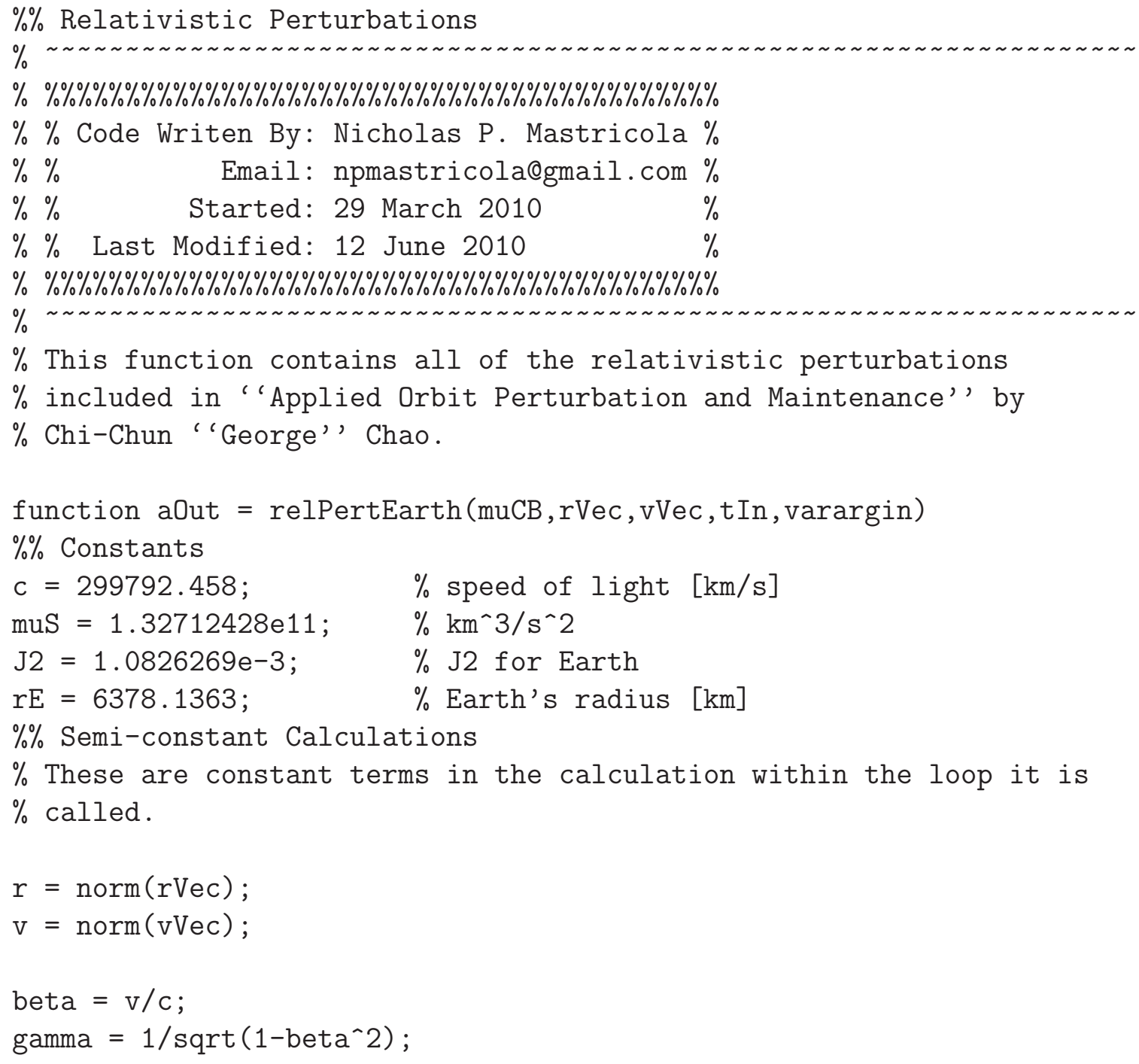




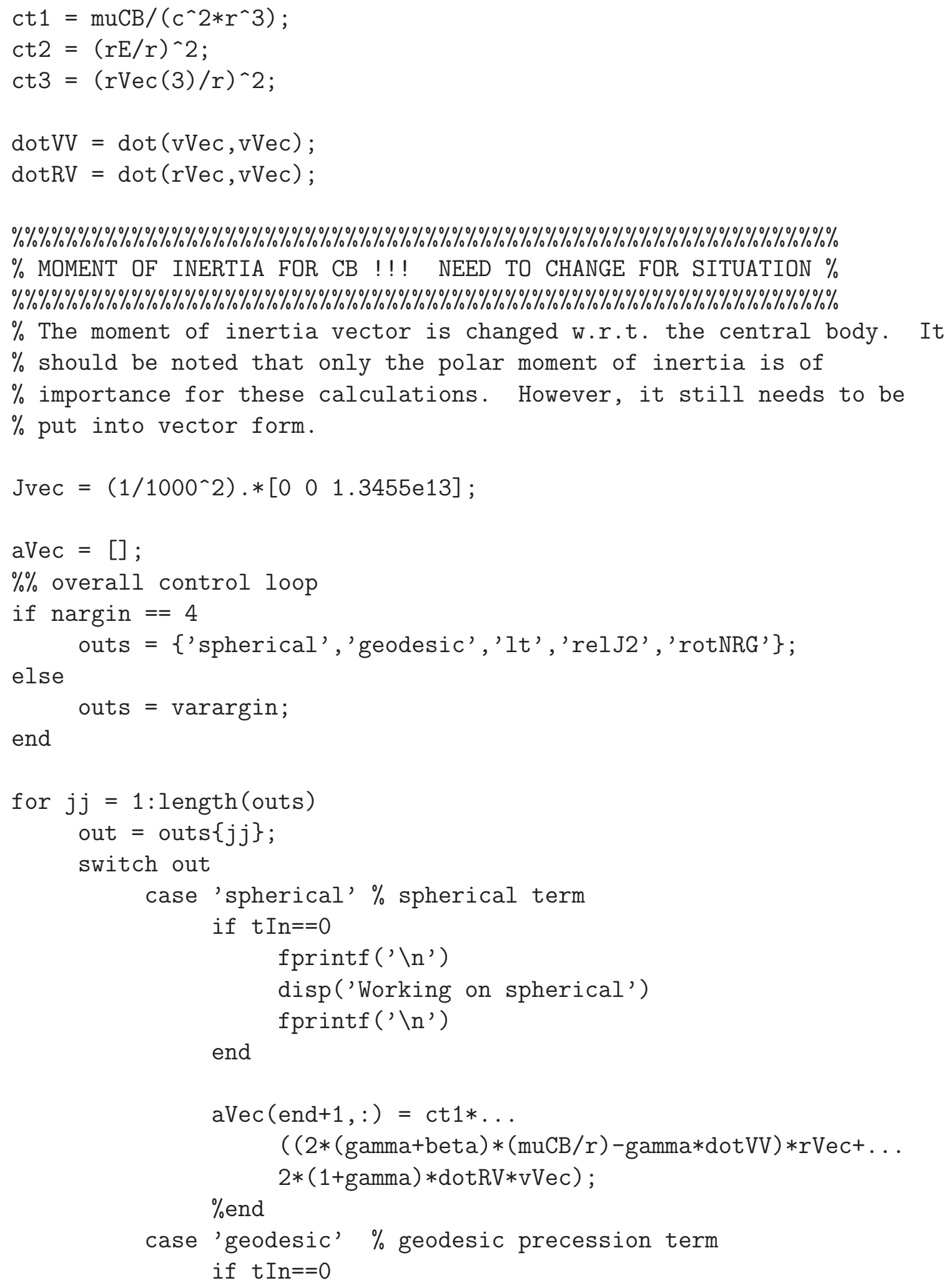




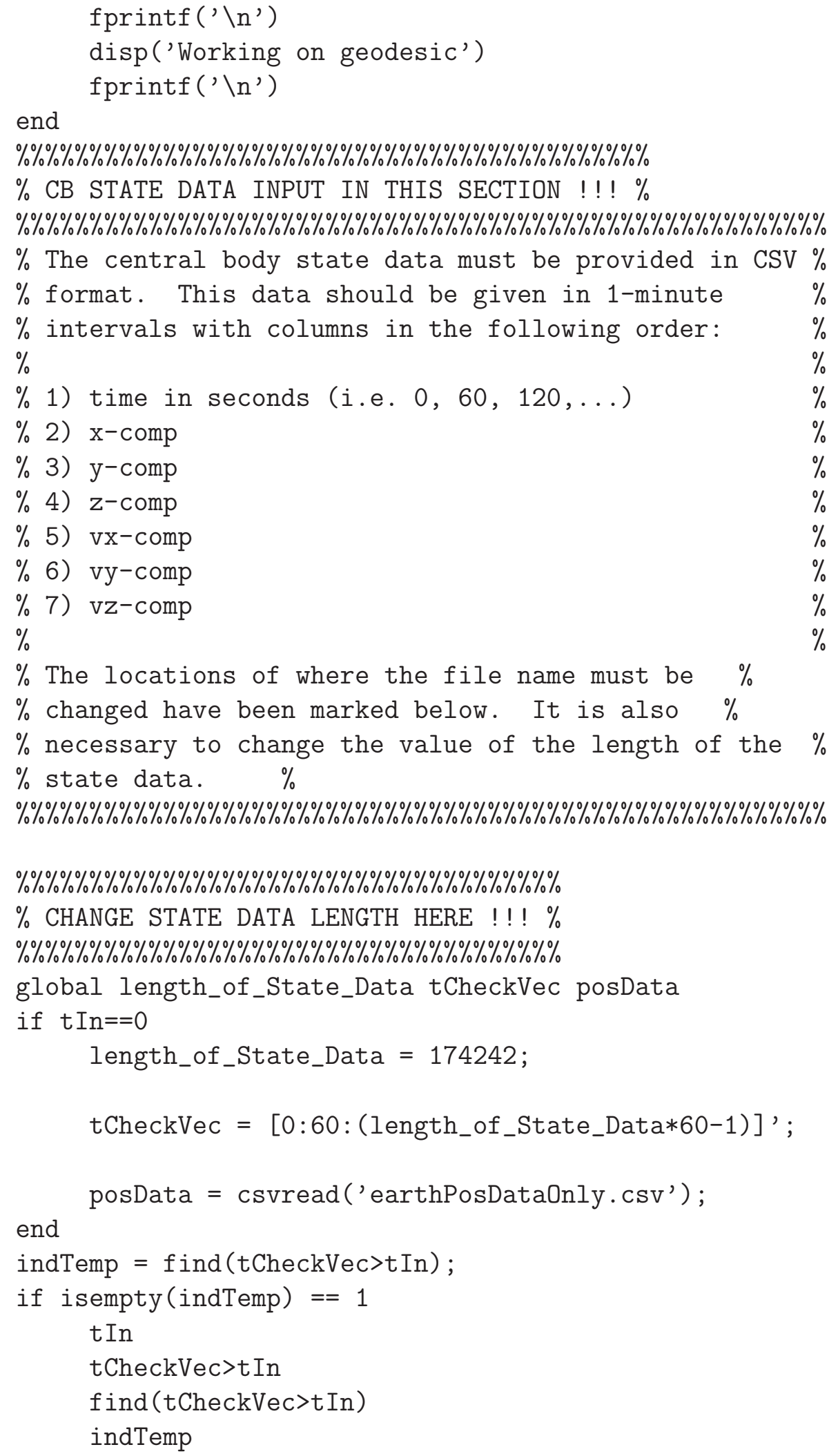




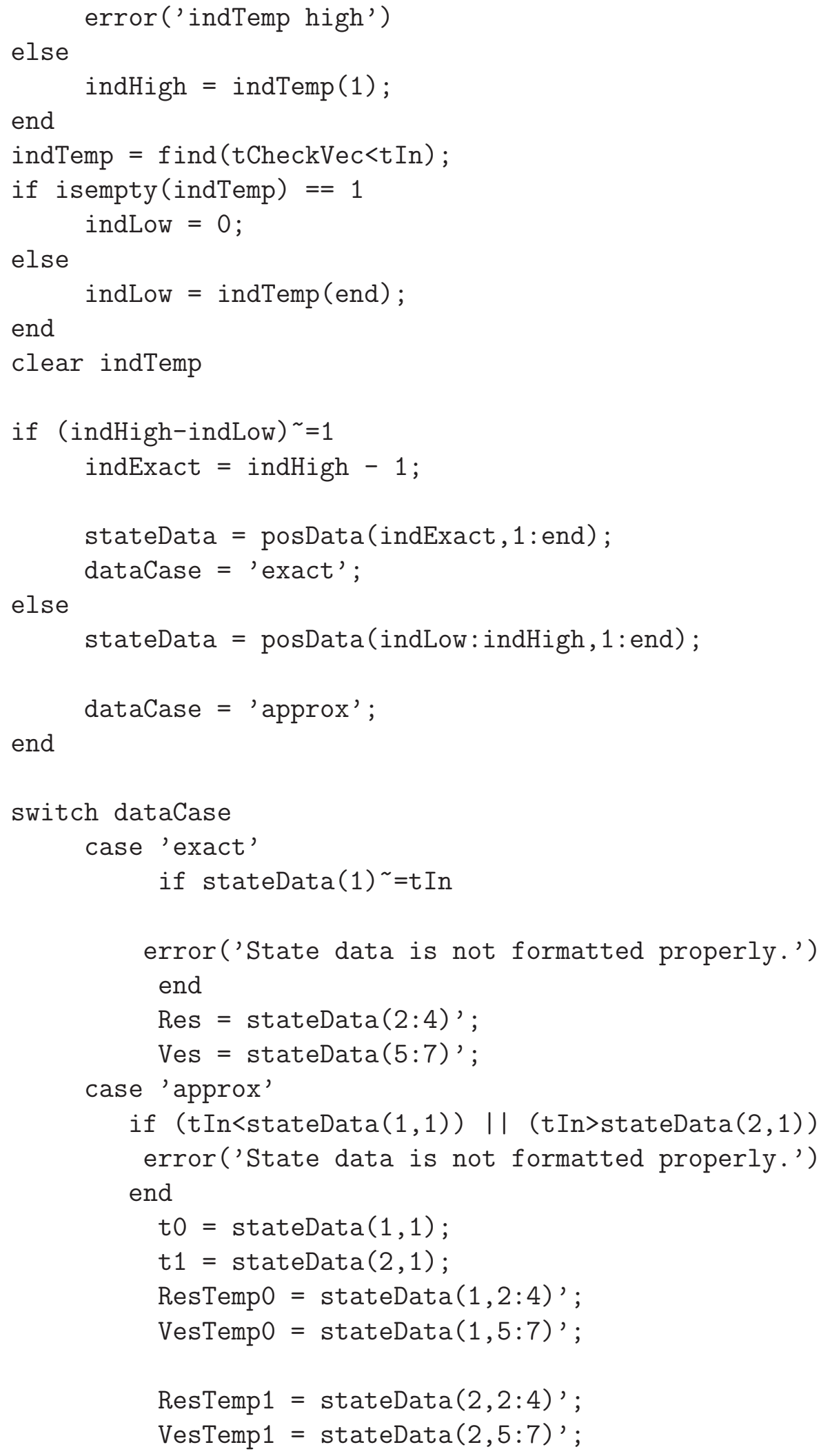




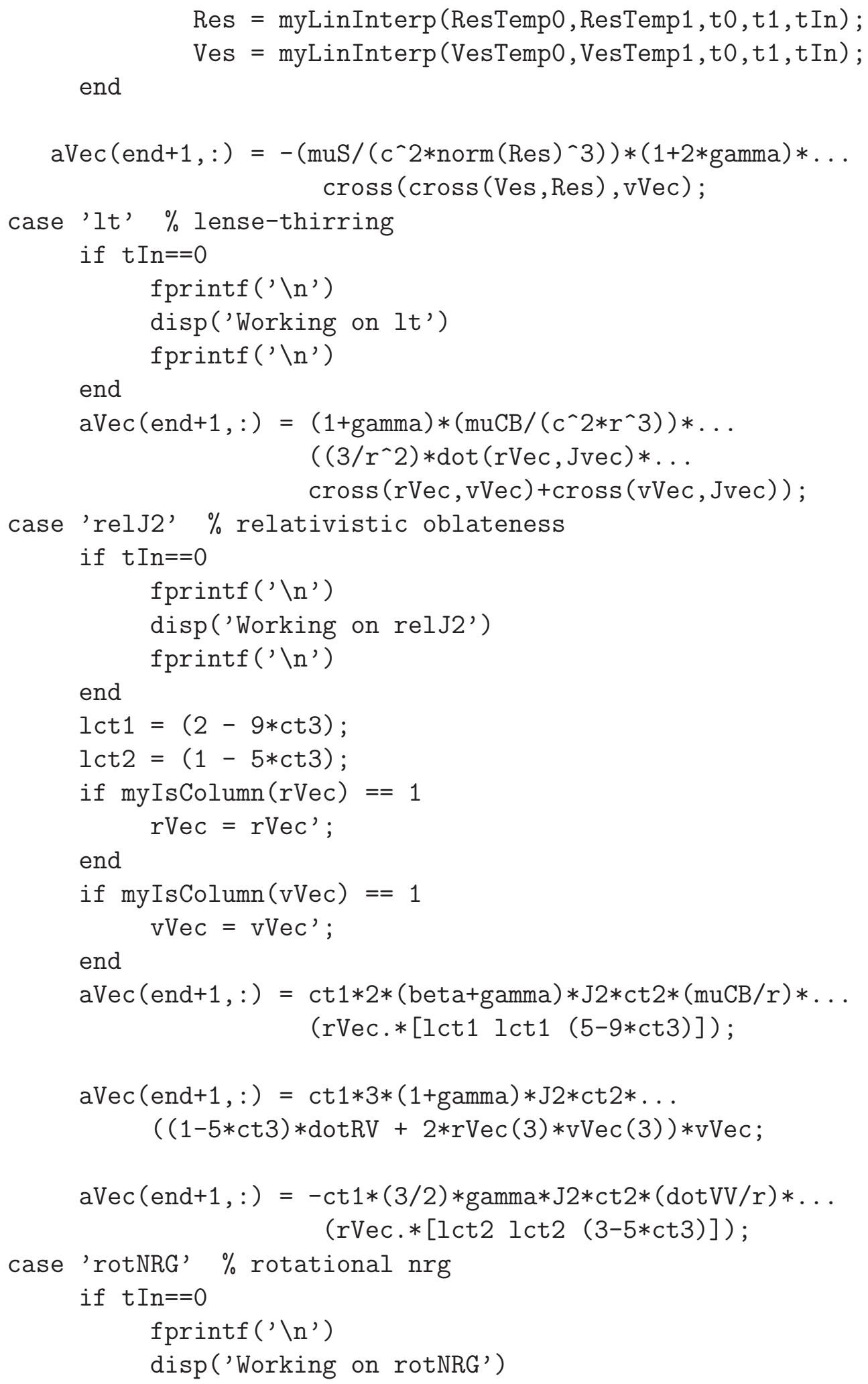




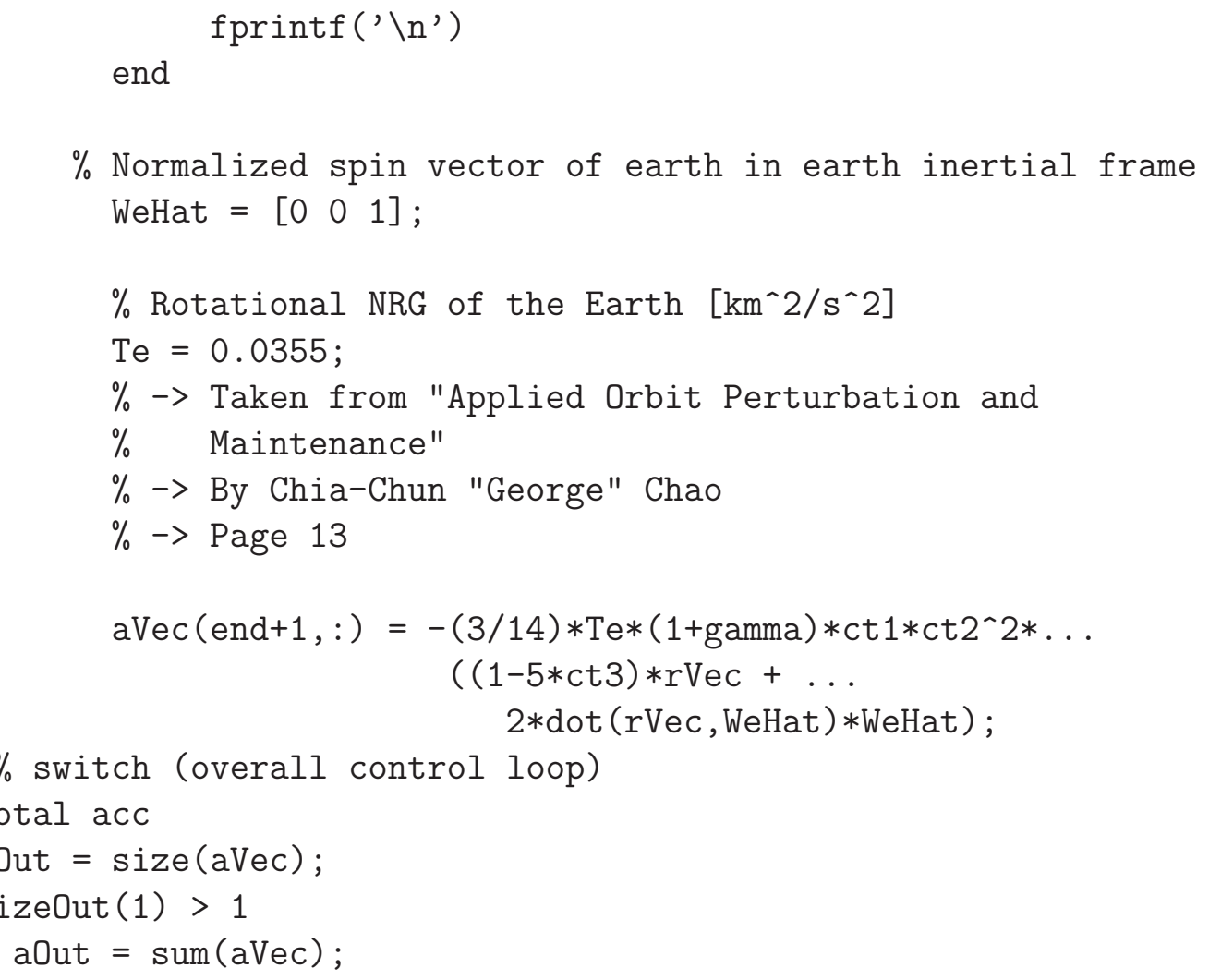




\section{Appendix $\mathrm{K}$}

\section{Relativistic Perturbation Data for Standard Case}

\section{K.1 Orbital Parameter Data}

The data presented in Figures K.1 to K.10 is summarized in Table K.1. In Table K.1, the abbreviations "Sec." and "Per." are used for secular and periodic, respectively, where necessary. Table K.1 is only representative of the graphical data. To aid in the analysis process, the minimum, maximum, mean, and median values of the orbital parameters' differences were examined. These values are seen in Table K.2.

Table K.1: Summarized Orbital Parameter Data for Standard Orbit

\begin{tabular}{|c|c|c|c|c|c|c|}
\hline & \multicolumn{5}{|c|}{ Perturbations } \\
\hline & & Spherical & $\begin{array}{l}\text { Lense-- } \\
\text { Thirring }\end{array}$ & $\begin{array}{l}\text { Relativistic } \\
\text { Oblateness }\end{array}$ & $\begin{array}{l}\text { Rotational } \\
\text { Energy }\end{array}$ & $\begin{array}{l}\text { Geodesic } \\
\text { Precession }\end{array}$ \\
\hline \multirow{2}{*}{$\theta$} & Variation & Sec. with Per. & Sec. with Per. & Sec. with Per. & Sec. with Per. & Sec. with Per. \\
\hline & Trend & Increasing & Increasing & Increasing & Increasing & Increasing \\
\hline \multirow[b]{2}{*}{$a$} & Variation & Sec. with Per. & Secular & Secular & Secular & Secular \\
\hline & Trend & Decreasing & Decreasing & Decreasing & Decreasing & Decreasing \\
\hline \multirow{2}{*}{$e$} & Variation & Secular & Sec. with Per. & Secular & Secular & Secular \\
\hline & Trend & Increasing & Increasing & Increasing & Increasing & Increasing \\
\hline \multirow[b]{2}{*}{$p$} & Variation & Secular & Secular & Secular & Secular & Secular \\
\hline & Trend & Decreasing & Decreasing & Decreasing & Decreasing & Decreasing \\
\hline \multirow{2}{*}{$\Omega$} & Variation & Indeterminate & Secular & Secular & Secular & Secular \\
\hline & Trend ${ }^{-}$ & Indeterminate & Increasing & Increasing & Decreasing & Increasing \\
\hline \multirow[b]{2}{*}{$\omega$} & Variation & Secular & Secular & Secular & Secular & Secular \\
\hline & Trend & Increasing & Decreasing & Decreasing & Decreasing & Decreasing \\
\hline \multirow[b]{2}{*}{$i$} & Variation & Indeterminate & Periodic & Secular & Secular & Secular \\
\hline & Trend & Indeterminate & $n / a^{-\cdots}$ & Increasing & Decreasing & Decreasing \\
\hline
\end{tabular}


Table K.2: COE Statistics for Standard Orbit Case

\begin{tabular}{|c|c|c|c|c|c|c|}
\hline & & \multicolumn{5}{|c|}{ Perturbations } \\
\hline & & Spherical & $\begin{array}{l}\text { Lense-- } \\
\text { Thirring }\end{array}$ & $\begin{array}{l}\text { Relativistic } \\
\text { Oblateness }\end{array}$ & $\begin{array}{l}\text { Rotational } \\
\text { Energy }\end{array}$ & $\begin{array}{l}\text { Geodesic } \\
\text { Precession }\end{array}$ \\
\hline \multirow{4}{*}{$\begin{array}{c}\theta \\
\text { (radians) }\end{array}$} & Min & $-2.3334 \mathrm{E}-6$ & $-7.5830 \mathrm{E}-7$ & -1.1757 E -10 & -1.1710 E -10 & -1.2114 E -10 \\
\hline & Max & $\overline{4} . \overline{6} \overline{7} \overline{\mathrm{E}} \overline{\mathrm{E}}-\overline{4}$ & $5.4042 \mathrm{E}-4$ & $5.3926 \mathrm{E}-4$ & $5 . \overline{4} \overline{0} \overline{7} \mathrm{E}-4$ & $5.4078 \mathrm{E}-\overline{4}$ \\
\hline & Mean & $4.8979 \overline{\mathrm{E}}-5$ & $6.14 \overline{3} 9 \mathrm{E}-5$ & $6.1383 \mathrm{E}-5$ & $6 . \overline{1} \overline{4} \overline{5}-5$ & $6.1566 \mathrm{E}-5$ \\
\hline & ' $\bar{M} \bar{e} \overline{d i a n}$ & $2 . \overline{4} \overline{0} 0 \overline{\mathrm{E}}-\overline{5}^{-}$ & $3.4007 \mathrm{E}-5$ & $3.4025 \mathrm{E}-5$ & $3 . \overline{1} \overline{1} \overline{\mathrm{E}}-5$ & $3.4126 \mathrm{E}-5$ \\
\hline \multirow{4}{*}{$\begin{array}{c}a \\
(\mathrm{~km})\end{array}$} & Min & -0.0010 & -0.0010 & -0.0010 & -0.0010 & -0.0010 \\
\hline & $\operatorname{Max}$ & $6.1560 \mathrm{E}-5$ & $8.4232 \mathrm{E}-6$ & $8.4599 \mathrm{E}-6$ & $8.4 \overline{2} 2 \overline{5} \overline{\mathrm{E}}-6$ & $8.4231 \mathrm{E}-6-$ \\
\hline & Mean & $-\overline{4} \overline{0} \overline{6} \overline{\mathrm{E}}-\overline{4}$ & $-5.1986 \mathrm{E}-4$ & $-\overline{1} \overline{1} \overline{4} \overline{9} \mathrm{E}-\overline{4}$ & $-5.1983 \mathrm{E}-4$ & $-\overline{19} 8 \overline{1} \mathrm{E}-\overline{4}$ \\
\hline & 'Mèdian & $-4 . \overline{0} \overline{4} 6 \mathrm{E}-4$ & $-5.1993 \mathrm{E}-4$ & $-5.1853 \mathrm{E}-4$ & $-5.2000 \mathrm{E}-4$ & $-5.1958 \mathrm{E}-\overline{4}^{-}$ \\
\hline \multirow{4}{*}{$\begin{array}{c}e \\
\text { (unitless) }\end{array}$} & Min & $-1.2613 \mathrm{E}-10$ & $-1.3160 \mathrm{E}-8$ & $-1.4417 \mathrm{E}-10$ & $-1.4500 \mathrm{E}-10$ & $-1.4518 \mathrm{E}-10$ \\
\hline & Max & $\overline{6} .22 \overline{9} \overline{\mathrm{E}}-\overline{8}^{--}$ & $2 . \overline{7} 8 \overline{7} \mathrm{E}-7$ & $6.0703 \mathrm{E}-8$ & $6.0551 \mathrm{E}-8$ & $6.0548 \mathrm{E}-8$ \\
\hline & Mean & $3 . \overline{1} \overline{0} \overline{\mathrm{E}}-\overline{8}$ & $1.8 \overline{2} \overline{5} \mathrm{E}-7$ & $3.0292 \mathrm{E}-8$ & $3 . \overline{0} \overline{1} \overline{6}-\bar{E}$ & $3.0205 \mathrm{E}-8$ \\
\hline & Médian & $3.169 \overline{8}-\overline{8}$ & $2.1 \overline{1} 60 \mathrm{E}-7$ & $3.0303 \mathrm{E}-\overline{8}^{-}$ & $3 . \overline{0} \overline{2} \overline{5} \mathrm{E}-\overline{8}$ & $3.0219 \mathrm{E}-8$ \\
\hline \multirow{4}{*}{$\begin{array}{c}p \\
(\mathrm{~km})\end{array}$} & Min & -0.0016 & -0.0042 & -0.0016 & -0.0016 & -0.0016 \\
\hline & Max & $2.71639 \mathrm{E}-5$ & $1.5839 \mathrm{E}-4$ & $5.9371 \mathrm{E}-6^{-}$ & $5 . \overline{9} 25 \overline{\mathrm{E}}-6$ & $6.0149 \mathrm{E}-6$ \\
\hline & ${ }^{-} \bar{M}$ ean & $-7.5808 \mathrm{E}-4$ & $-0.002 \overline{6}^{-}$ & $-\overline{7} \overline{\bar{y}} \overline{2} \overline{2} \overline{\mathrm{E}}-\overline{4}$ & $-7.7937 \mathrm{E}-4$ & $-\overline{7} \overline{7} \overline{2} \overline{3} \overline{\mathrm{E}}-\overline{4}$ \\
\hline & Mèdian & $-7.5815 \mathrm{E}-4$ & -0.0028 & $-\overline{7} . \overline{9} \overline{5} \overline{\mathrm{E}}-\overline{4}$ & $-7.7965 \mathrm{E}-4$ & $-7.796 \overline{6} \mathrm{E}-\overline{4}$ \\
\hline \multirow{4}{*}{$\begin{array}{c}\Omega \\
\text { (radians) }\end{array}$} & Min & $-7.1054 \mathrm{E}-15$ & 0.0000 & 0.0000 & $-6.7648 \mathrm{E}-11$ & 0.0000 \\
\hline & Max & $1.5321 \mathrm{E}-14^{-}$ & $5.3970 \mathrm{E}-4$ & $1.3594 \mathrm{E}-\overline{9}^{-}$ & $0 . \overline{0} 0 \overline{0} \overline{0}^{-\cdots}$ & $2.5303 \mathrm{E}-8$ \\
\hline & ${ }^{-} \bar{M}^{-}{ }^{-}{ }^{-}$ & $4 . \overline{3} 448 \mathrm{E}-15$ & $2.6992 \mathrm{E}-4$ & $6 . \overline{9} \overline{9} \overline{8}-10$ & $-\overline{3} . \overline{3} \overline{4} \overline{3} \mathrm{E}-1 \overline{1}$ & $1.2686 \mathrm{E}-8$ \\
\hline & 'Médian & $4.3854 \mathrm{E}-15$ & $2.6 \overline{9} \overline{3} \mathrm{E}-\overline{4}$ & $6 . \overline{0} 0 \overline{0} \overline{\mathrm{E}}-10$ & $-3 . \overline{3} 8 \overline{4} \overline{\mathrm{E}}-1 \overline{1}$ & $1.2702 \mathrm{E}-8$ \\
\hline \multirow{4}{*}{$\begin{array}{c}\omega \\
\text { (radians) }\end{array}$} & Min & 0.0000 & -0.0014 & $-5.2862 \mathrm{E}-7$ & $-5.2775 \mathrm{E}-7$ & $-5.3210 \mathrm{E}-7$ \\
\hline & Max & $4.7132 \mathrm{E}-6$ & $0.0000^{--}$ & $8.6 \overline{6} 58 \mathrm{E}-11$ & $8.6431 \mathrm{E}-11$ & $8.967 \overline{7}^{-}-1 \overline{1}^{-}$ \\
\hline & Mean & $2.3567 \mathrm{E}-6$ & $-68666 \mathrm{E}-4$ & $-2 . \overline{6} \overline{9} \overline{4} \overline{\mathrm{E}}-\overline{7}$ & $-2.6351 \mathrm{E}-7$ & $-2.6568 \mathrm{E}-7^{-}$ \\
\hline & Médian & $2 . \overline{3} 5 \overline{6} 8 \mathrm{E}-\overline{6}^{--}$ & $-6.8673 \mathrm{E}-\overline{4}$ & $-\overline{2} . \overline{6} \overline{0} \overline{7} \mathrm{E}-\overline{7}$ & $-2.6362 \mathrm{E}-7$ & $-2.6580 \mathrm{E}-\overline{7}^{-}$ \\
\hline \multirow{4}{*}{$\begin{array}{c}i \\
\text { (radians) }\end{array}$} & Min & $-1.0658 \mathrm{E}-14$ & $-6.8509 \mathrm{E}-8$ & $-1.8407 \mathrm{E}-13$ & $-2.7207 \mathrm{E}-12$ & $-1.6775 \mathrm{E}-9$ \\
\hline & Max & $5.4401 \mathrm{E}-15$ & $6.1042 \mathrm{E}-8$ & $2 . \overline{0} \overline{6} \overline{3}-11$ & $8.8818 \mathrm{E}-15$ & $\overline{6} \overline{0} \overline{2} \overline{4}-\overline{1} \overline{3}^{-}$ \\
\hline & ${ }^{-} \bar{M} \overline{e a n}^{-}$ & $-2.0923 \mathrm{E}-1 \overline{5}^{-}$ & $2 . \overline{3} \overline{0} \overline{3}-8$ & $1.0 \overline{1} \overline{5} \overline{\mathrm{E}}-1 \overline{1}$ & $-\overline{1} . \overline{3} 5 \overline{3} \overline{\mathrm{E}}-\overline{1} \overline{2}$ & $-8.4086 \mathrm{E}-10$ \\
\hline & ' Médian & $-\overline{2} 1094 \mathrm{E}-1 \overline{5}$ & $2.2503 \mathrm{E}-8$ & $1.0 \overline{1} 5 \overline{6}-11$ & $-1 . \overline{3} 557 \mathrm{E}-1 \overline{2}$ & $-8 . \overline{4} 2 \overline{2} \mathrm{E}-10$ \\
\hline
\end{tabular}



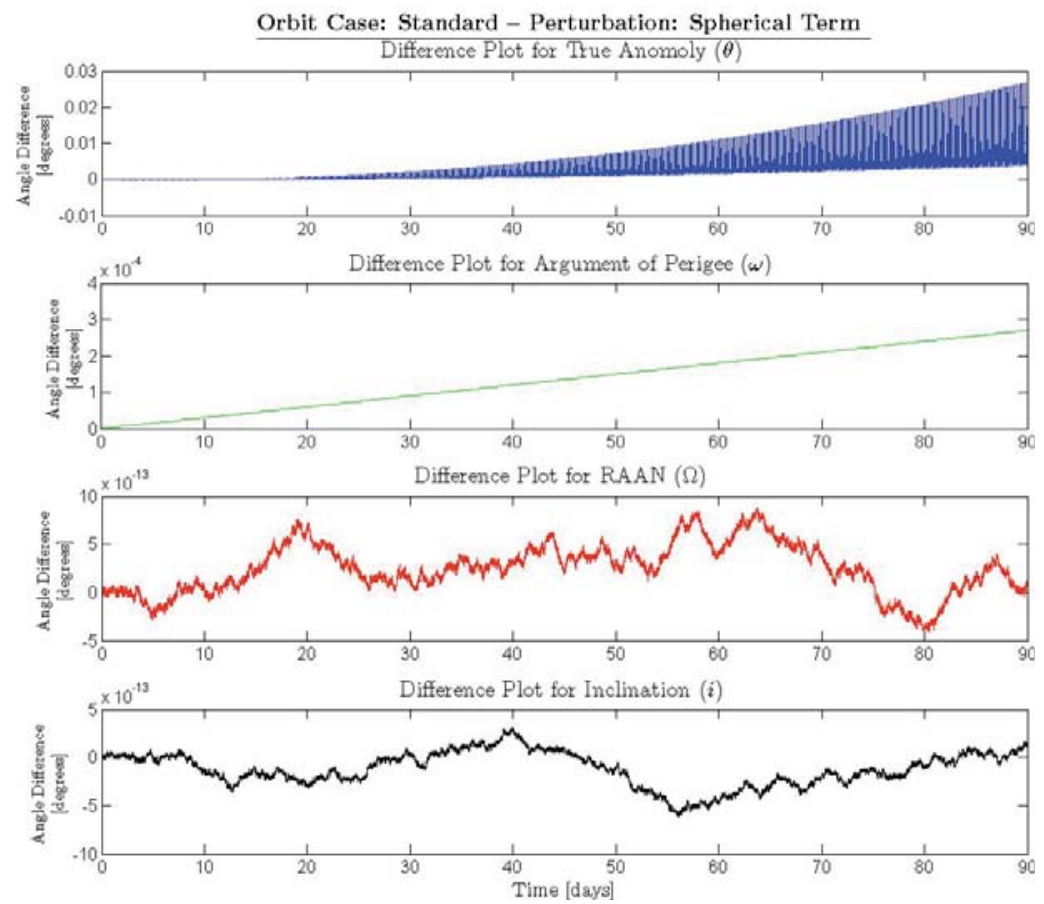

Figure K.1: Standard Orbit Angular Differences for Spherical Perturbation versus Time
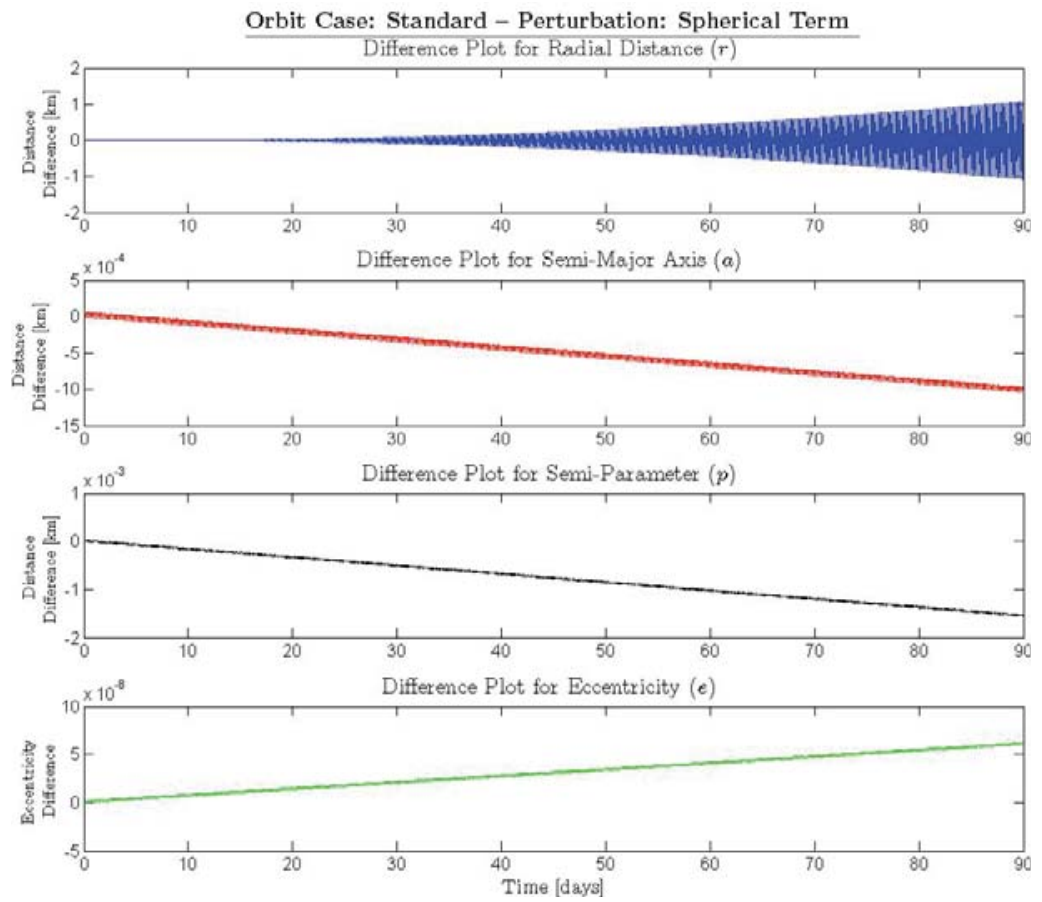

Figure K.2: Standard Orbit Distance Differences for Spherical Perturbation versus Time 

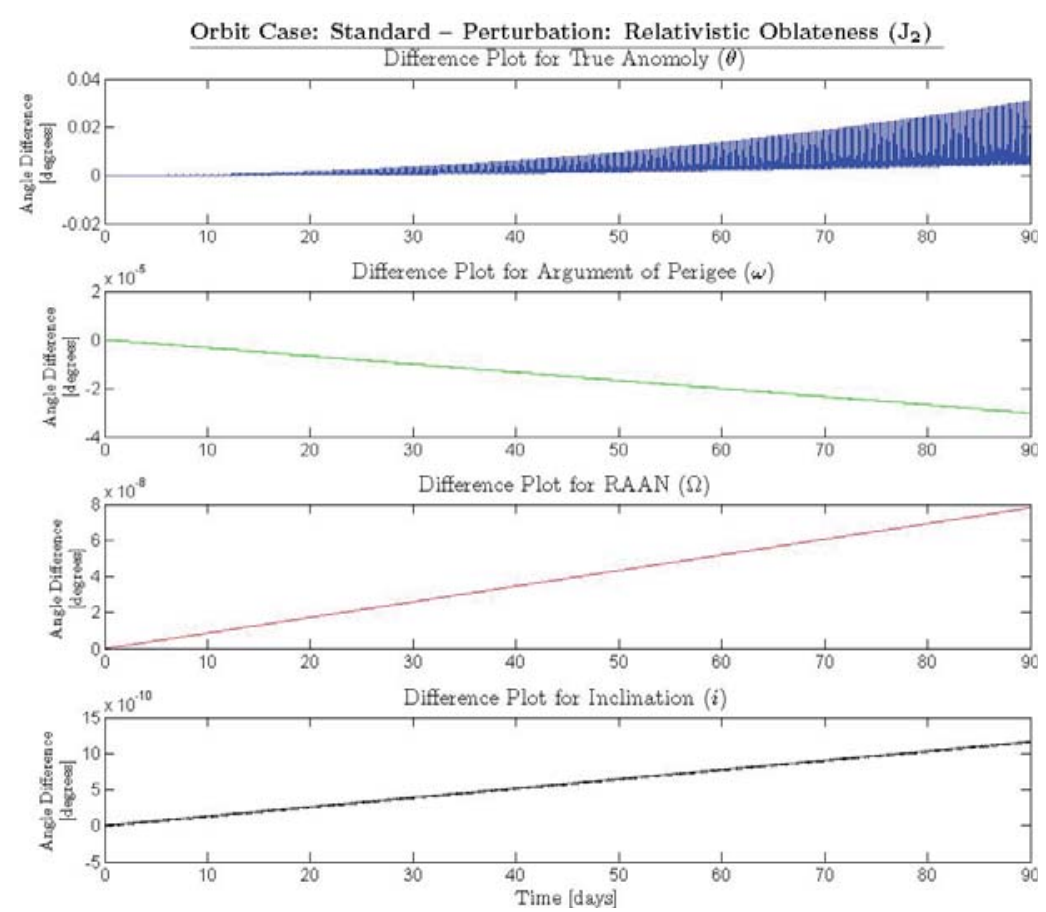

Figure K.3: Standard Orbit Angular Differences for Relativistic Oblateness versus Time
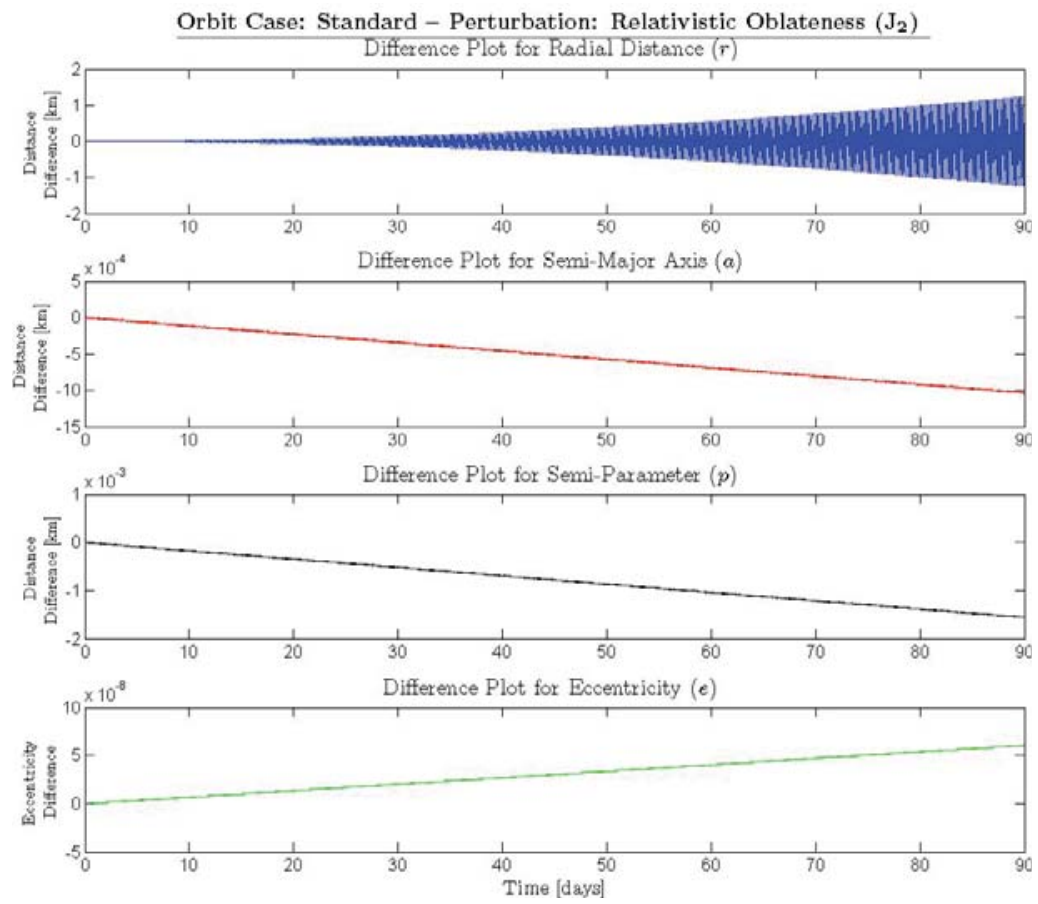

Figure K.4: Standard Orbit Distance Differences for Relativistic Oblateness versus Time 

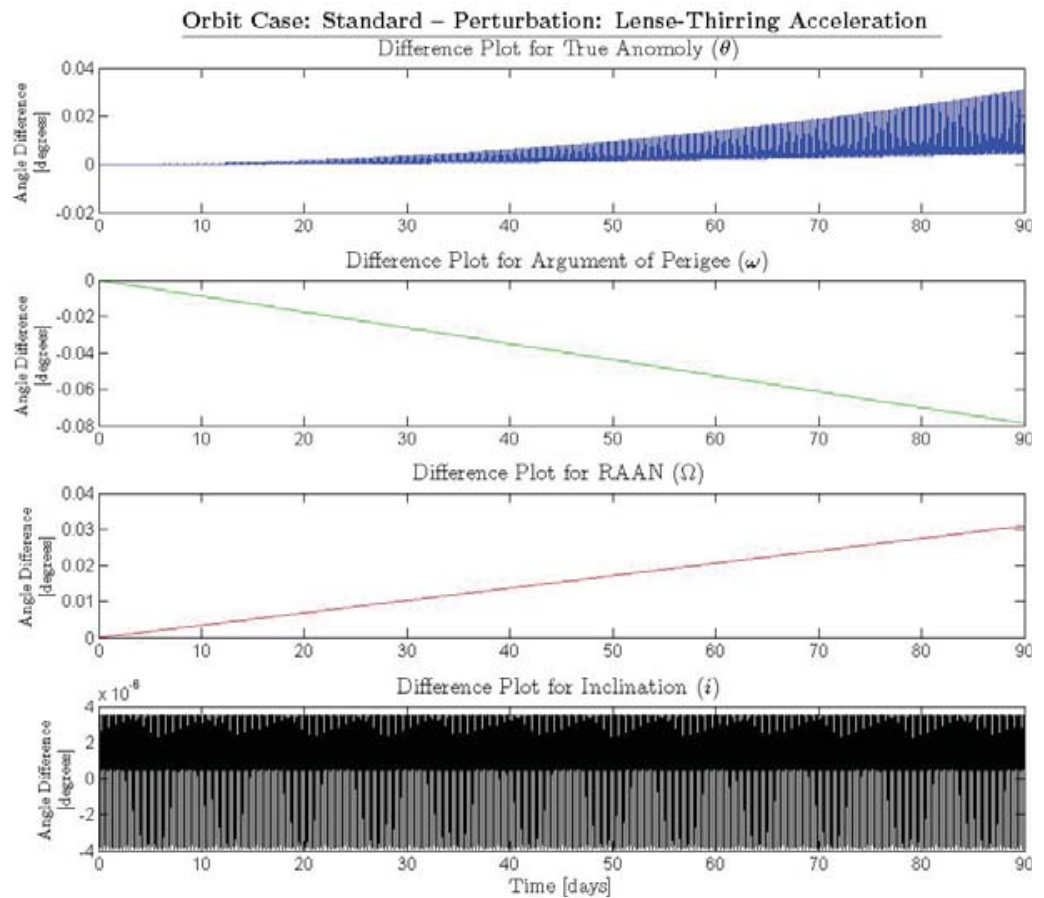

Figure K.5: Standard Orbit Angular Differences for Lense-Thirring versus Time
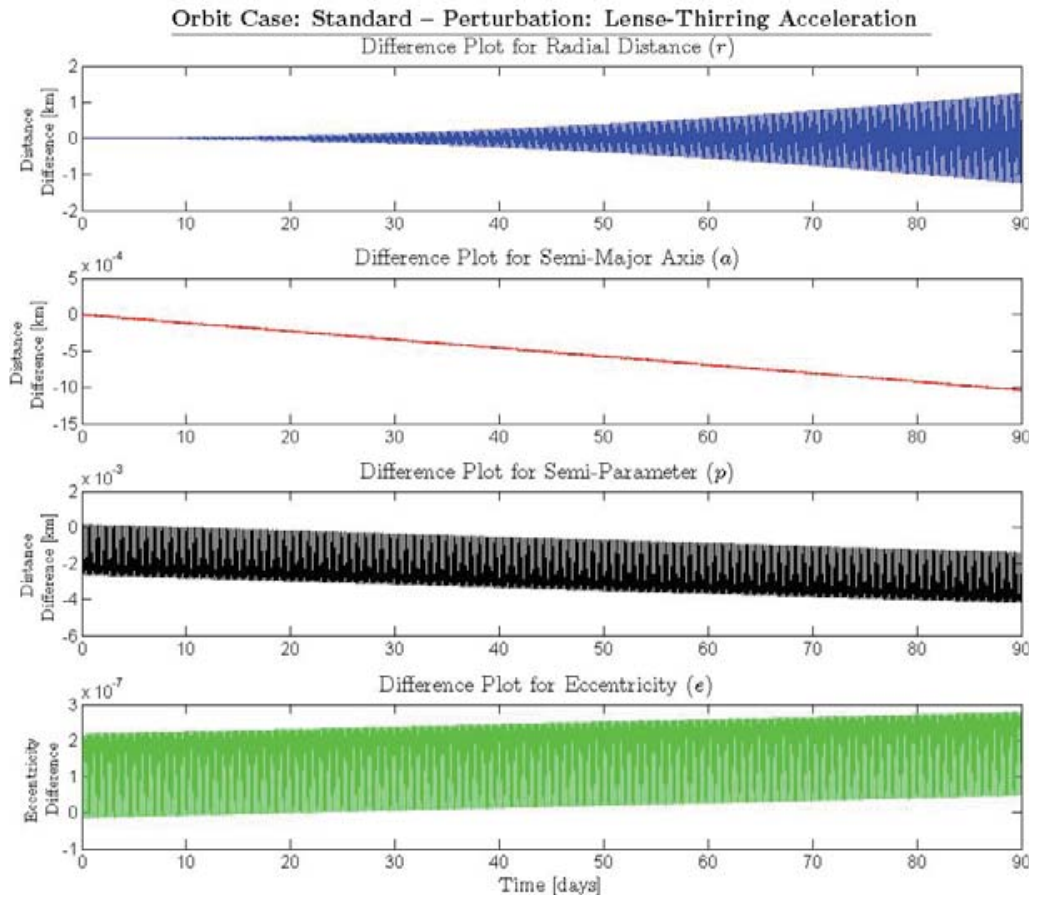

Figure K.6: Standard Orbit Distance Differences for Lense-Thirring versus Time 

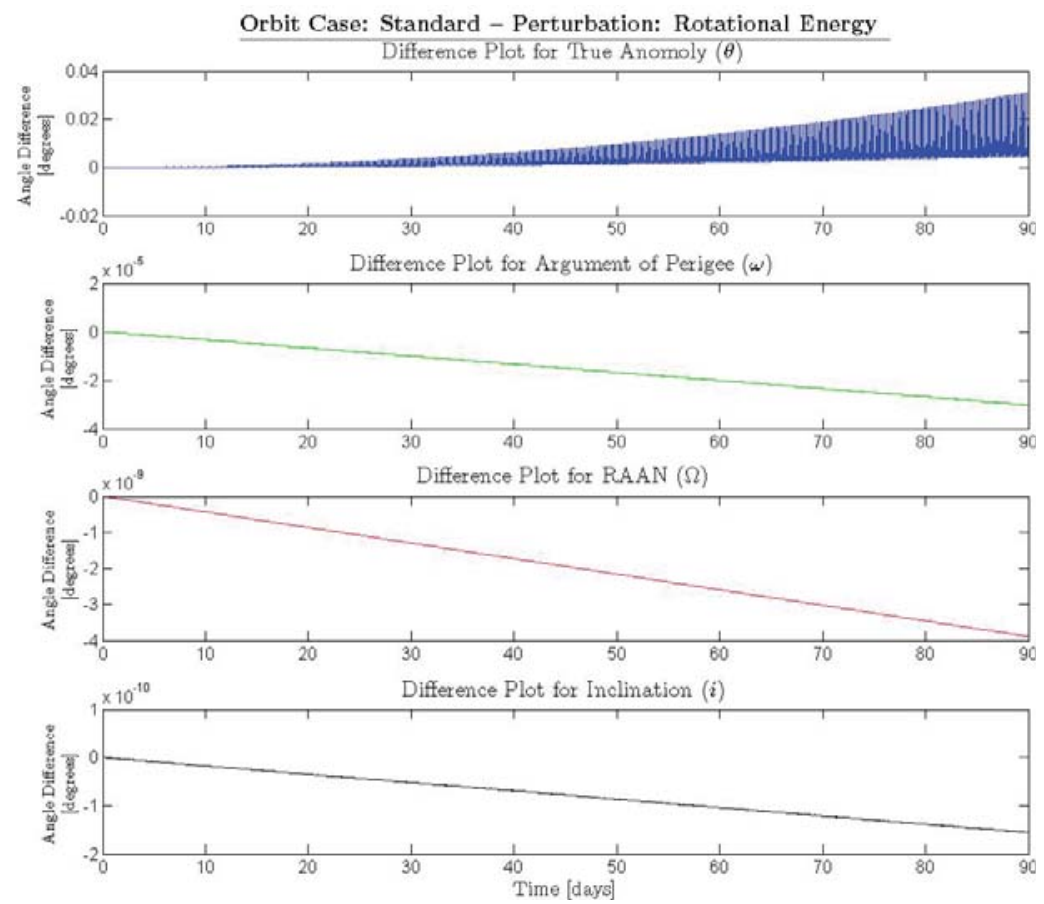

Figure K.7: Standard Orbit Angular Differences for Rotational Energy versus Time
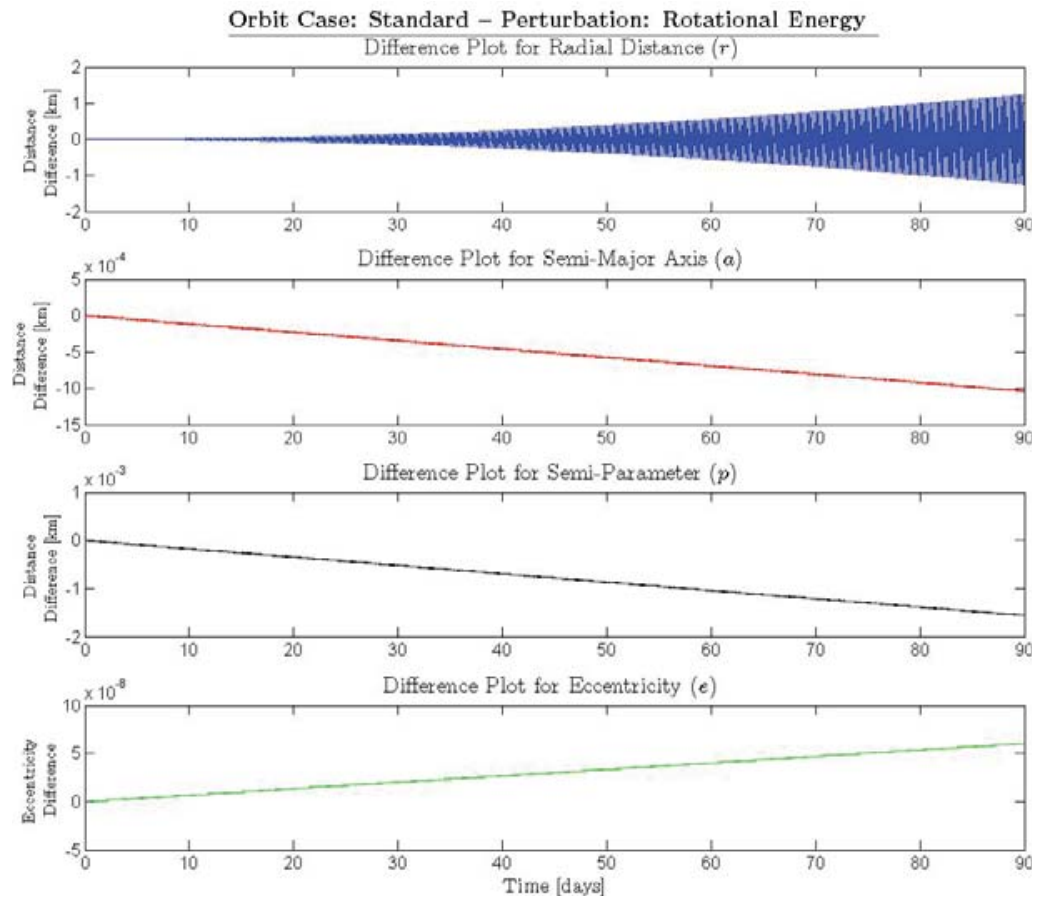

Figure K.8: Standard Orbit Distance Differences for Rotational Energy versus Time 

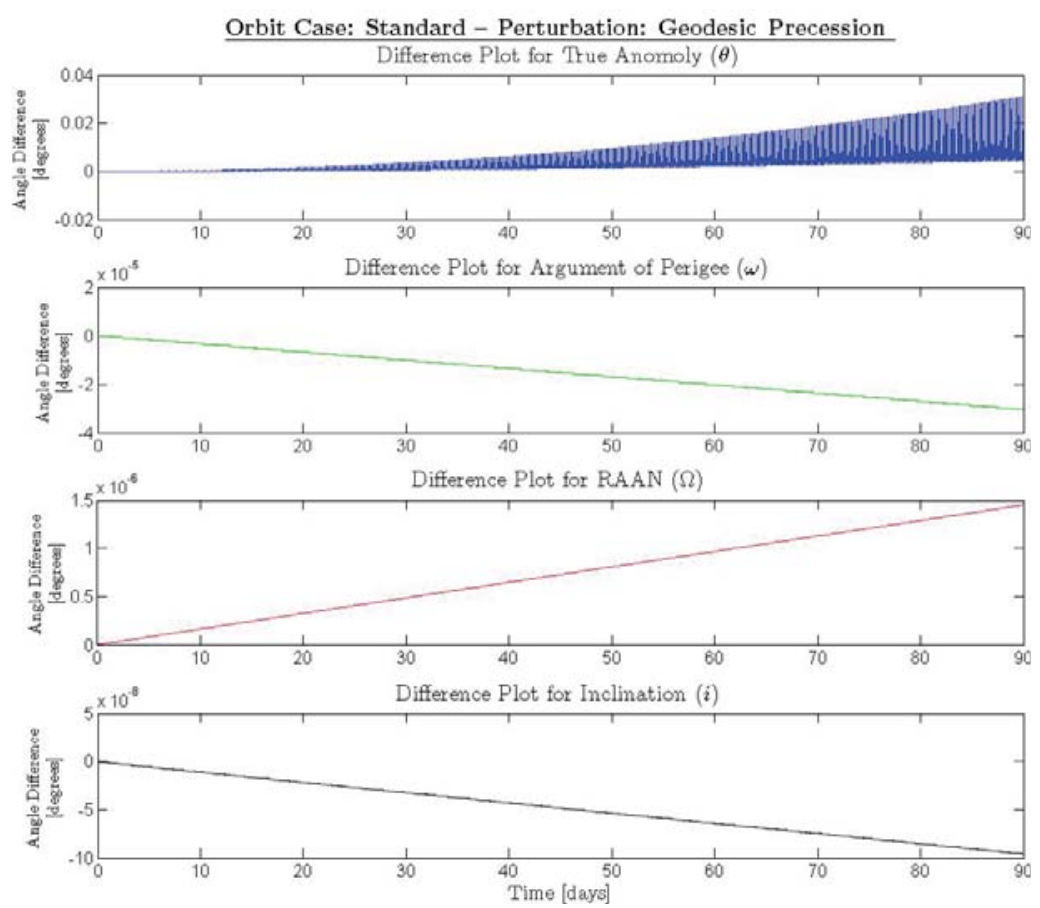

Figure K.9: Standard Orbit Angular Differences for Geodesic versus Time
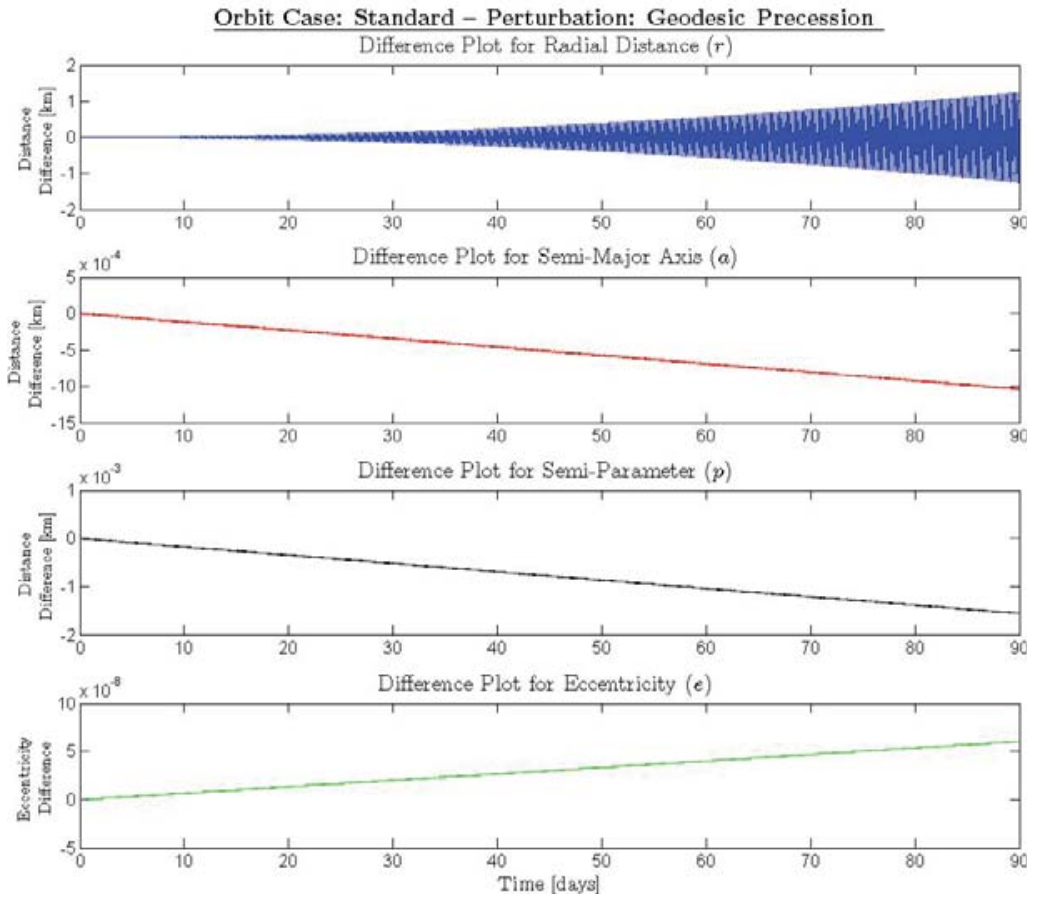

Figure K.10: Standard Orbit Distance Differences for Geodesic versus Time 


\section{K.2 Specific Force Data}

Figures K.11 through K.15 show the specific forces induced by the relativistic perturbations on the standard case orbit. All of the components and the magnitudes do not experience secular variations. This data is summarized in Table K.3, where the minimum, maximum, mean, and median values of the specific force magnitudes are given.

Table K.3: Specific Force Magnitude Data Summary for Standard Case

\begin{tabular}{|c|c|c|c|c|c|c|}
\hline & \multicolumn{5}{|c|}{ Perturbations } & \multirow[b]{2}{*}{ Units } \\
\hline & Spherical & $\begin{array}{l}\text { Lense-- } \\
\text { Thirring }\end{array}$ & $\begin{array}{l}\text { Relativistic } \\
\text { Oblateness }\end{array}$ & $\begin{array}{l}\text { Rotational } \\
\text { Energy }\end{array}$ & $\begin{array}{l}\text { Geodesic } \\
\text { Precession }\end{array}$ & \\
\hline Min & $3.4830 \mathrm{E}-7$ & $4.9138 \mathrm{E}-11$ & $1.0676 \mathrm{E}-16$ & $2.0045 \mathrm{E}-18$ & $1.4906 \mathrm{E}-14$ & \multirow{4}{*}{$\frac{\mathrm{m}}{\mathrm{s}^{2}}$} \\
\hline $\operatorname{Max}$ & $5.2509 \mathrm{E}-12$ & $2.6990 \mathrm{E}-9$ & $1.6818 \mathrm{E}-14$ & $6.7816 \mathrm{E}-16$ & $4.2298 \mathrm{E}-14$ & \\
\hline Mean & $1.745 \mathrm{E}-12$ & $5.3501 \mathrm{E}-10$ & $2.2605 \mathrm{E}-15$ & $8.0623 \mathrm{E}-15$ & $2.6379 \mathrm{E}-14$ & \\
\hline Median & $9.5935 \mathrm{E}-13$ & $1.3613 \mathrm{E}-10$ & $2.6582 \mathrm{E}-16$ & $6.5495 \mathrm{E}-18$ & $2.4894 \mathrm{E}-14$ & \\
\hline
\end{tabular}
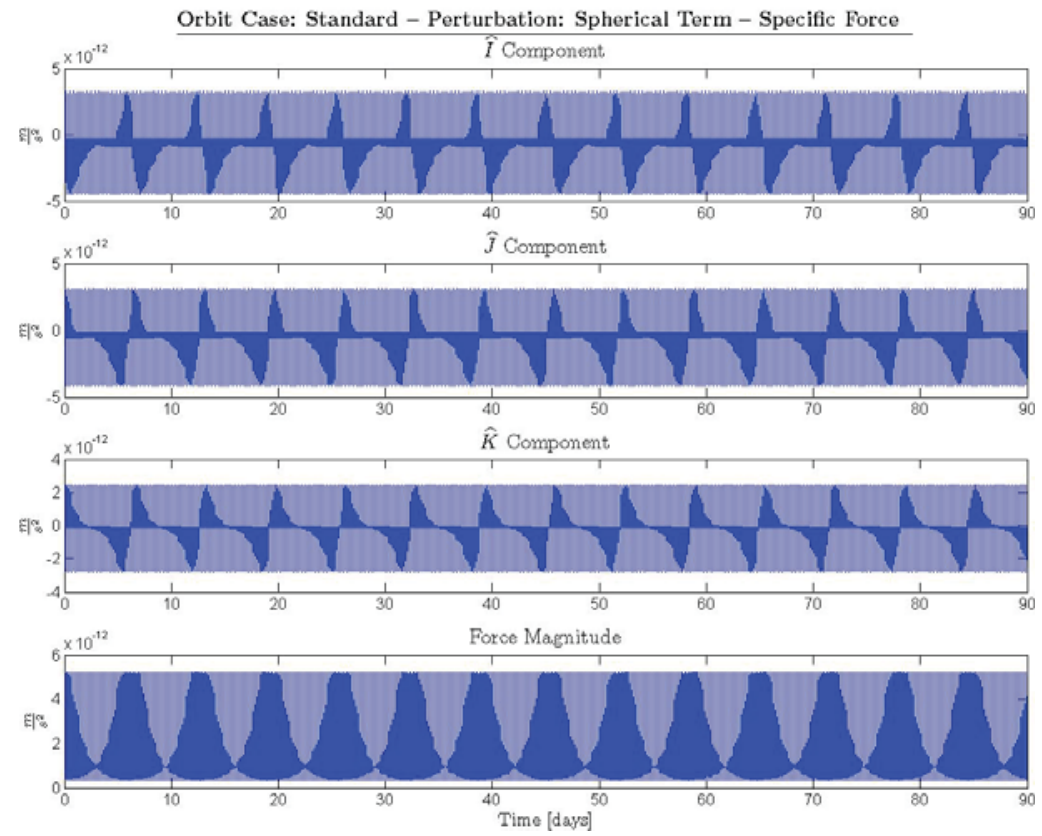

Figure K.11: Standard Orbit Specific Force for Spherical Perturbation versus Time 

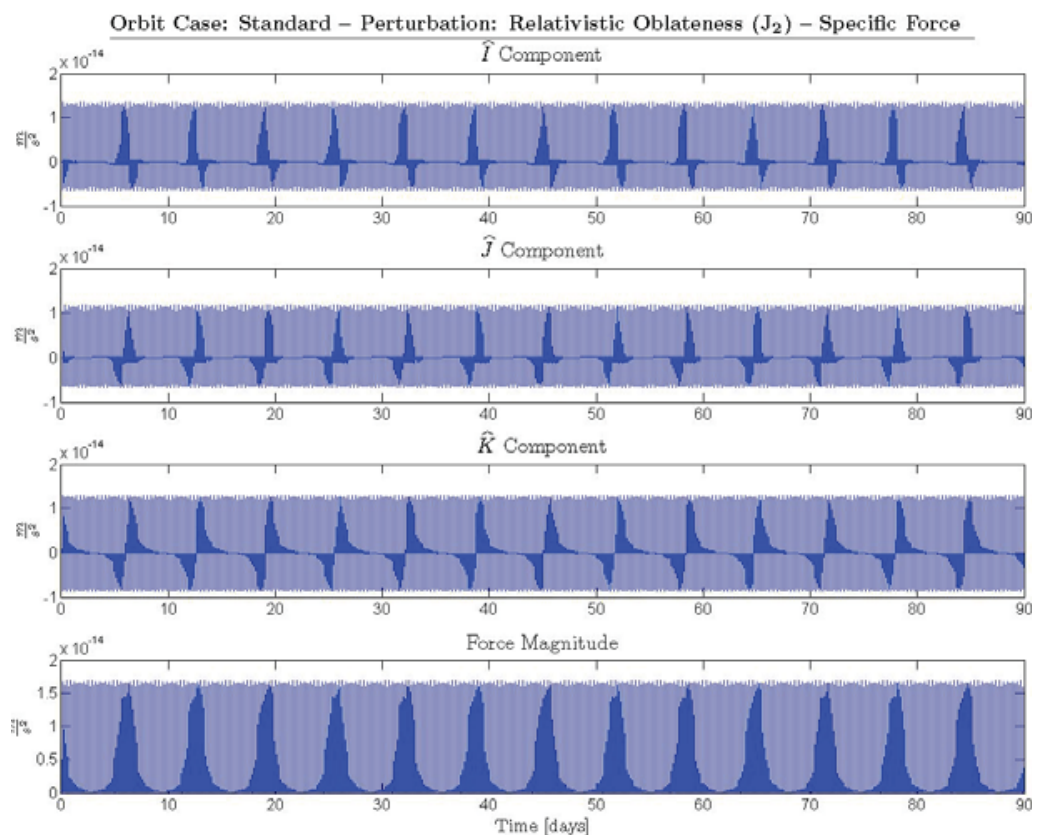

Figure K.12: Standard Orbit Specific Force for Relativistic Oblateness versus Time
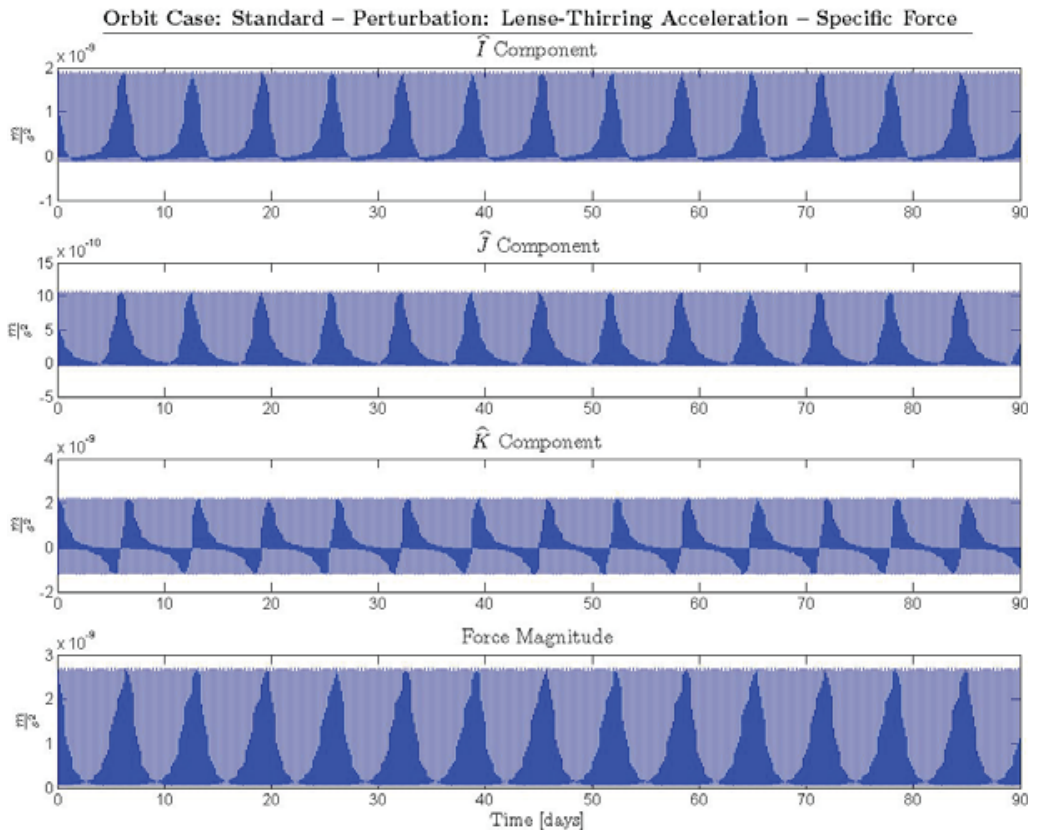

Figure K.13: Standard Orbit Specific Force for Lense-Thirring versus Time 

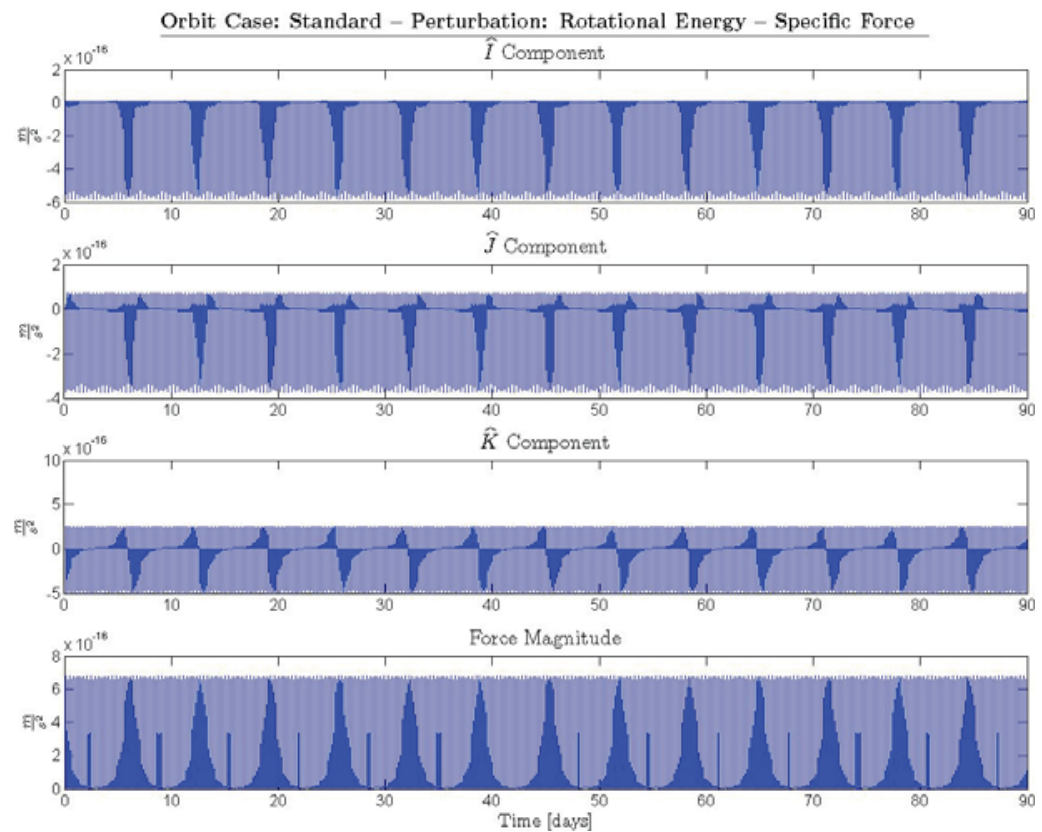

Figure K.14: Standard Orbit Specific Force for Rotational Energy versus Time
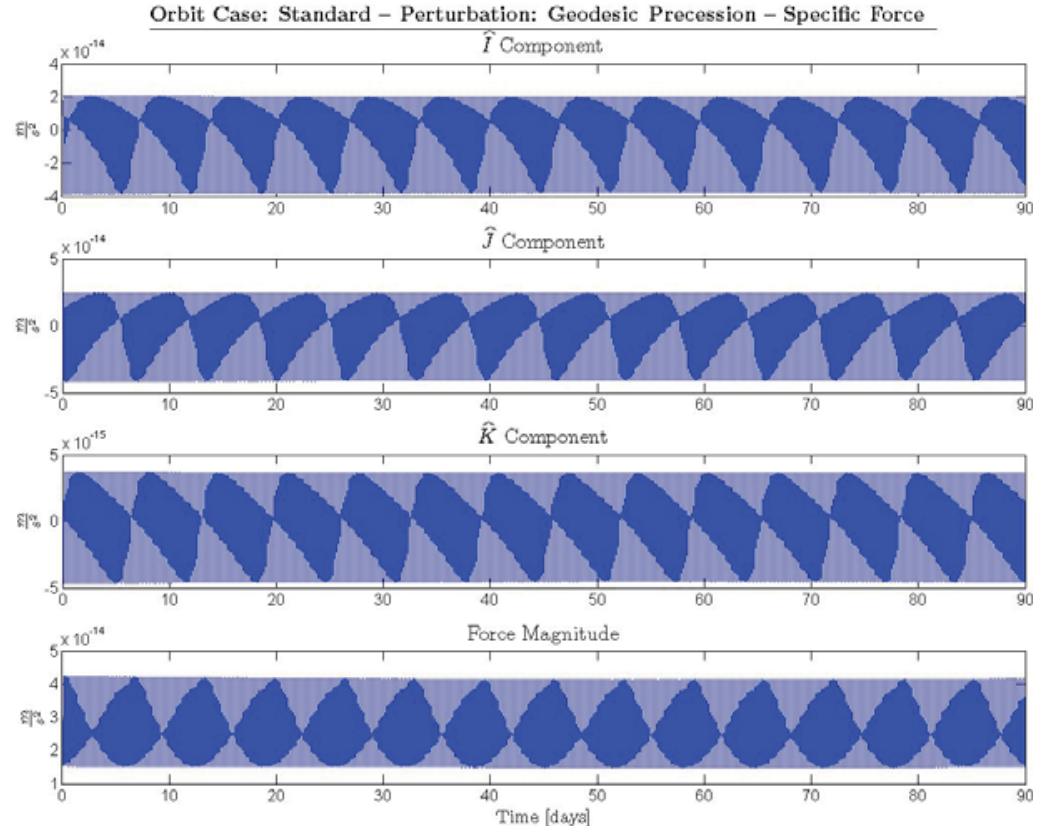

Figure K.15: Standard Orbit Specific Force for Geodesic versus Time 


\section{Appendix L}

\section{Relativistic Perturbation Data for Molniya Case}

\section{L.1 Orbital Parameter Data}

Figures L.1 to L.10 show the difference plots for the raw orbital parameter data that was generated for the Molniya orbit. This data is summarized in Tables L.1 and L.2.

Table L.1: Summarized Orbital Parameter Data for Molniya Orbit

\begin{tabular}{|c|c|c|c|c|c|c|}
\hline & \multicolumn{5}{|c|}{ Perturbations } \\
\hline & & Spherical & $\begin{array}{l}\text { Relativistic } \\
\text { Oblateness }\end{array}$ & $\begin{array}{l}\text { Lense-- } \\
\text { Thirring }\end{array}$ & $\begin{array}{l}\text { Rotational } \\
\text { Energy }\end{array}$ & $\begin{array}{l}\text { Geodesic } \\
\text { Precession }\end{array}$ \\
\hline \multirow{2}{*}{$\theta$} & Variation & Sec. with Per. & Sec. with Per. & Sec. with Per. & Sec. with Per. & Sec. with Per. \\
\hline & Trend & Increasing & Increasing & Increasing & Increasing & Increasing \\
\hline \multirow[b]{2}{*}{$a$} & Variation & Sec. with Per. & Secular & Secular & Secular & Secular \\
\hline & Trend & Decreasing & Decreasing & Decreasing & Decreasing & Decreasing \\
\hline \multirow{2}{*}{$e$} & Variation & Sec. with Per. & Secular & Sec. with Per. & Secular & Secular \\
\hline & Trend & Decreasing & Decreasing & Decreasing & Decreasing & Decreasing \\
\hline \multirow[b]{2}{*}{$p$} & Variation & Sec. with Per. & Secular & Sec. with Per. & Secular & Secular \\
\hline & Trend & Decreasing & Decreasing & Decreasing & Decreasing & Decreasing \\
\hline \multirow{2}{*}{$\Omega$} & Variation & Indeterminate & Indeterminate & Indeterminate & Indeterminate & Indeterminate \\
\hline & Trend & Indeterminate & Indeterminate & Indeterminate & Indeterminate & Indeterminate \\
\hline \multirow[b]{2}{*}{$\omega$} & Variation & Indeterminate & Indeterminate & Indeterminate & Indeterminate & Indeterminate \\
\hline & Trend & Indeterminate & Indeterminate & Indètermināte & Indéterminate & Indeterminate \\
\hline \multirow{2}{*}{$i$} & Variation & Indeterminate & Periodic & Periodic & Indeterminate & Secular \\
\hline & Trend & Indeterminate & n/a & n $7 \mathrm{a}^{-\cdots}$ & Indeterminate & Decreasing \\
\hline
\end{tabular}


Table L.2: COE Statistics for Molniya Orbit Case

\begin{tabular}{|c|c|c|c|c|c|c|}
\hline & & \multicolumn{5}{|c|}{ Perturbations } \\
\hline & & Spherical & $\begin{array}{l}\text { Lense-- } \\
\text { Thirring }\end{array}$ & $\begin{array}{l}\text { Relativistic } \\
\text { Oblateness }\end{array}$ & $\begin{array}{l}\text { Rotational } \\
\text { Energy }\end{array}$ & $\begin{array}{l}\text { Geodesic } \\
\text { Precession }\end{array}$ \\
\hline \multirow{4}{*}{$\begin{array}{c}\theta \\
\text { (radians) }\end{array}$} & Min & -8.6104 E -6 & $-1.9335 \mathrm{E}-7$ & $-6.9146 \mathrm{E}-10$ & $-6.9007 \mathrm{E}-10$ & $-6.9794 \mathrm{E}-10$ \\
\hline & $\operatorname{Max}$ & $3.7735 \mathrm{E}-4$ & $5.1827 \mathrm{E}-4$ & $5.1826 \mathrm{E}-4$ & $5.18 \overline{3} 4 \mathrm{E}-4$ & $5.1848 \mathrm{E}-4$ \\
\hline & ${ }^{-} \bar{M}^{-} \bar{e}^{-}$ & $\overline{1} . \overline{2} \overline{3} \overline{\mathrm{E}}^{-}-\overline{5}^{-}$ & $2 . \overline{2} \overline{0} 2 \mathrm{E}-5$ & $2.2210 \mathrm{E}-5^{-}$ & $\overline{2} \overline{2} \overline{2} \overline{1} \overline{\mathrm{E}}-\overline{5}$ & $2 . \overline{2} 224 \mathrm{E}-5$ \\
\hline & ${ }^{-} \bar{M} \bar{e} \bar{d} \bar{i} \bar{a}$ - & $\overline{3} . \overline{0} \overline{5} \overline{\mathrm{E}}-\overline{6}$ & $7.7 \overline{2} \overline{6}-\bar{E}-6$ & $7.7300 \mathrm{E}-\overline{6}^{-}$ & $7 . \overline{3} \overline{2} \overline{2}-\overline{6}$ & $7.7363 \mathrm{E}-6$ \\
\hline \multirow{4}{*}{$\begin{array}{c}a \\
(\mathrm{~km})\end{array}$} & Min & -0.0021 & -0.0021 & -0.0021 & -0.0021 & -0.0021 \\
\hline & Max & $\overline{2} . \overline{0} \overline{5} \overline{\mathrm{E}}-\overline{4}$ & $3.9057 \mathrm{E}-5$ & $\overline{3} . \overline{2} 02 \mathrm{E}-5$ & $3 . \overline{9} \overline{5} 5 \overline{\mathrm{E}}^{-}-5$ & $3.9056 \mathrm{E}-5$ \\
\hline & Mean & $-7 . \overline{2} 3 \overline{3} \mathrm{E}-4$ & $-0.0010^{--}$ & $-0 . \overline{0} \overline{1} \overline{0}^{---}$ & $-0.0010^{--}$ & $-0.00 \overline{1} \overline{0}^{-\cdots}$ \\
\hline & Median & $-7.8279 \mathrm{E}-4$ & -0.0010 & $-0.0 \overline{0} \overline{1} 0^{-}$ & -0.0010 & $-0.0010^{-}$ \\
\hline \multirow{4}{*}{$\begin{array}{c}e \\
\text { (unitless) }\end{array}$} & Min & -8.3166 E -9 & $-1.0842 \mathrm{E}-8$ & $-8.5419 \mathrm{E}-9$ & $-8.5457 \mathrm{E}-9$ & $-8.5538 \mathrm{E}-9$ \\
\hline & Max & $2.8121 \mathrm{E}-9^{-}$ & $5.1263 \mathrm{E}-8$ & $4 . \overline{6} \overline{9} \overline{6} \mathrm{E}-10$ & $4.6364 \mathrm{E}-10$ & $4 . \overline{6} 30 \overline{8} \mathrm{E}-1 \overline{0}$ \\
\hline & Mean & $-1 . \overline{1} \overline{8} 0-\overline{\mathrm{E}}-9$ & $\overline{3} \overline{9} \overline{1} \overline{9} \overline{9}^{6}-\overline{8}$ & $-\overline{4} . \overline{0} \overline{8} \overline{5}-\overline{\mathrm{E}}-\overline{-}$ & $-4.0802 \mathrm{E}-9$ & $-\overline{4} .0 \overline{3} 0{ }^{-}-\bar{y}^{-}$ \\
\hline & ${ }^{-} \bar{M}$ édiān & $-1.7 \overline{1} 64 \mathrm{E}-9$ & $4.3742 \mathrm{E}-8$ & $-4.0 \overline{0} \overline{6} \overline{\mathrm{E}}-\overline{9}$ & $-4.0784 \mathrm{E}-9$ & $-\overline{4} .0 \overline{0} 0 \overline{\mathrm{E}}-\overline{9}^{-}$ \\
\hline \multirow{4}{*}{$\begin{array}{c}p \\
(\mathrm{~km})\end{array}$} & Min & $-7.4606 \mathrm{E}-4$ & -0.0027 & $-7.6181 \mathrm{E}-4$ & $-7.6187 \mathrm{E}-4$ & $-7.6122 \mathrm{E}-4$ \\
\hline & Max & $6.2 \overline{4} 81 \mathrm{E}-\overline{5}$ & $1.0 \overline{2} \overline{9} \mathrm{E}-4$ & $1.7735 \mathrm{E}-5$ & $\overline{1} \overline{7} \overline{2} \overline{0} \mathrm{E}-\overline{-}$ & $1.8289 \mathrm{E}-5$ \\
\hline & Mean & $-3 . \overline{3} 500 \mathrm{E}-4$ & $-0.0020^{--}$ & $-\overline{3} . \overline{4} \overline{2} \overline{9} \mathrm{E}-\overline{4}$ & $-3.7431 \mathrm{E}-4$ & $-\overline{3} . \overline{3} 8 \overline{\mathrm{E}}-\overline{4}^{-}$ \\
\hline & ' M̄édiā̄o- & $-3 . \overline{3} \overline{4} 85 \mathrm{E}-\overline{4}$ & -0.0021 & $-3 . \overline{4} \overline{4} \overline{8} \overline{\mathrm{E}}-\overline{4}$ & $-3.7439 \mathrm{E}-4$ & $-\overline{3} . \overline{7} \overline{8} \overline{6} \mathrm{E}-\overline{\mathrm{A}}^{-}$ \\
\hline \multirow{4}{*}{$\begin{array}{c}\Omega \\
\text { (radians) }\end{array}$} & Min & -6.2832 & -6.2832 & -6.2832 & 0.0000 & -6.2832 \\
\hline & Max & $\overline{6} \overline{2} \overline{2} \overline{8} \overline{3} \overline{2}^{-}$ & $1.3523 \mathrm{E}-4$ & ${ }^{-} \overline{0} 0000^{-}$ & $6 . \overline{2} \overline{8} \overline{3} \overline{2}^{-}$ & $2.1073 \mathrm{E}-8$ \\
\hline & ${ }^{-} \bar{M}^{--}{ }^{--}{ }^{-}$ & $4 . \overline{1} \overline{1} \overline{9} \overline{9}^{-}$ & -0.1221 & $-\overline{0} \overline{1} \overline{2} \overline{2} \overline{2}^{-}$ & $6.1 \overline{6} \overline{0} \overline{8}^{-}$ & ${ }^{-0} \overline{1} \overline{1} \overline{2} \overline{2}^{---}$ \\
\hline & Médian & $6 . \overline{2} \overline{8} \overline{2} \overline{2}^{-}$ & $6.5718 \mathrm{E}-5$ & $\overline{0} 0 \overline{0} 00^{-}$ & $6 . \overline{2} 8 \overline{3} \overline{2}^{--}$ & $\overline{0} \overline{0} \overline{0} \overline{0} \overline{0}^{--}$ \\
\hline \multirow{4}{*}{$\begin{array}{c}\omega \\
\text { (radians) }\end{array}$} & Min & -6.2832 & $-1.0872 \mathrm{E}-6$ & -6.2832 & -6.2832 & 0.0000 \\
\hline & Max & $1.3658 \mathrm{E}-6 \overline{1}$ & 6.2832 & $6.2832^{\cdots}$ & $6 . \overline{2} \overline{3} 2^{---}$ & $-\overline{6} . \overline{2} \overline{8} \overline{3} \overline{2}^{-}$ \\
\hline & $\bar{M}$ ean & $-4.8411 \mathrm{E}-4$ & 6.2824 & $7.2713 \mathrm{E}-4$ & $7.27 \overline{1} \overline{\mathrm{E}}-4$ & 0.0015 \\
\hline & ${ }^{-} \bar{M} \bar{e} \bar{d} \bar{i}{ }^{-}$ & $-\overline{6} \overline{8} \overline{1} \overline{3} \mathrm{E}-\overline{7}$ & $6 . \overline{2} 831$ & $-\overline{1} \overline{1} \overline{3} \overline{9} \mathrm{E}-\overline{8}^{-}$ & $-6.1439 \mathrm{E}-8$ & $-\overline{5} . \overline{3} \overline{2} \overline{\mathrm{E}} \mathrm{E}-\overline{8}^{-}$ \\
\hline \multirow{4}{*}{$\begin{array}{c}i \\
\text { (radians) }\end{array}$} & Min & $-3.3307 \mathrm{E}-15$ & $-4.1462 \mathrm{E}-8$ & $-5.0848 \mathrm{E}-14$ & $-1.2434 \mathrm{E}-14$ & -2.4881 E -9 \\
\hline & Max & $2.6645 \mathrm{E}-15$ & $1.3478 \mathrm{E}-7$ & $1.58 \overline{9} \overline{\mathrm{E}}-1 \overline{3}$ & $1.4433 \mathrm{E}-14$ & $1 . \overline{5} \overline{8} \overline{5} \mathrm{E}-\overline{1} \overline{2}^{-}$ \\
\hline & Mean & $-3.5104 \mathrm{E}-1 \overline{7}$ & $1.0 \overline{3} 05 \mathrm{E}-7$ & $1 . \overline{2} \overline{1} \overline{9} \mathrm{E}-1 \overline{3}$ & $-\overline{3} . \overline{4} \overline{3} \overline{\mathrm{E}}-\overline{1} \overline{5}$ & $-1.2510 \mathrm{E}-\overline{9}^{-}$ \\
\hline & Médian & $0.0 \overline{0} 0 \overline{0}$ & $1.0278 \mathrm{E}-7$ & $1 . \overline{2} \overline{6} 8 \overline{\mathrm{E}}-1 \overline{3}$ & $-\overline{3} 3 \overline{3} \overline{7} \mathrm{E}-\overline{1} \overline{5}$ & $-\overline{1} . \overline{2} 5 \overline{3} \mathrm{E}-\overline{9}^{-}$ \\
\hline
\end{tabular}



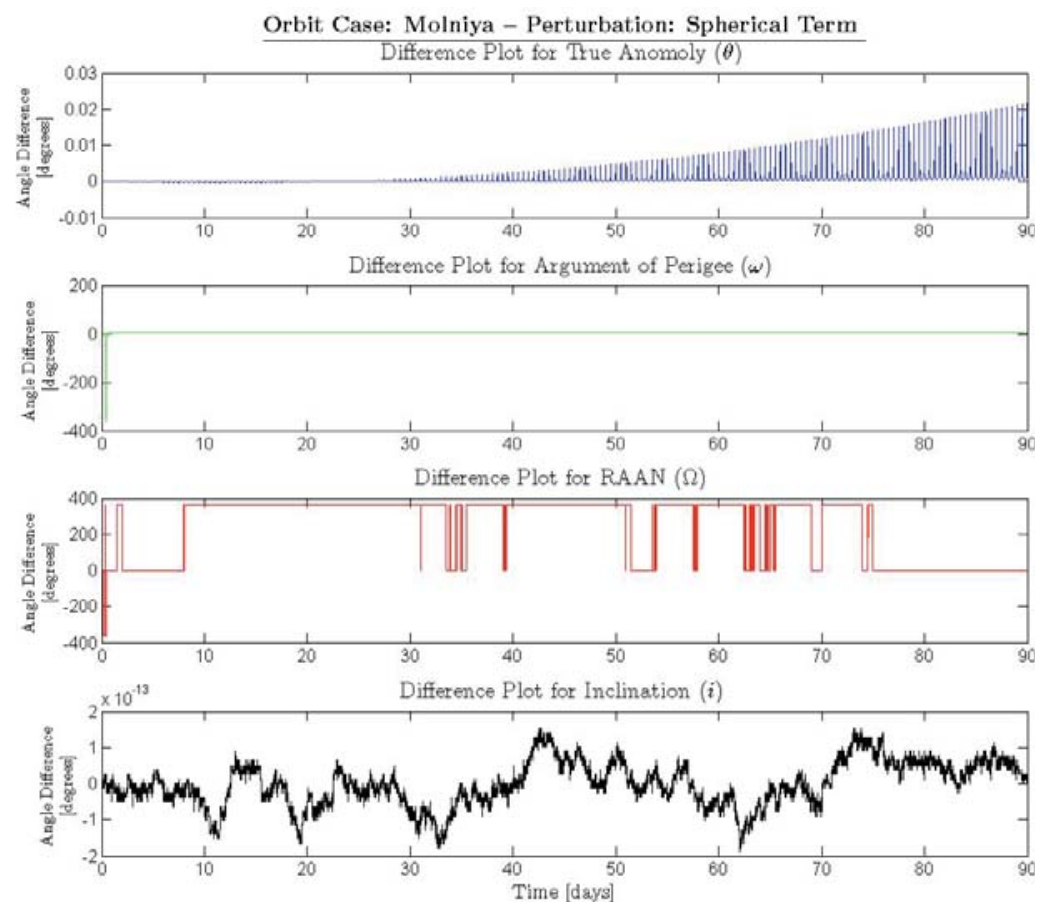

Figure L.1: Molniya Orbit Angular Differences for Spherical Perturbation versus Time
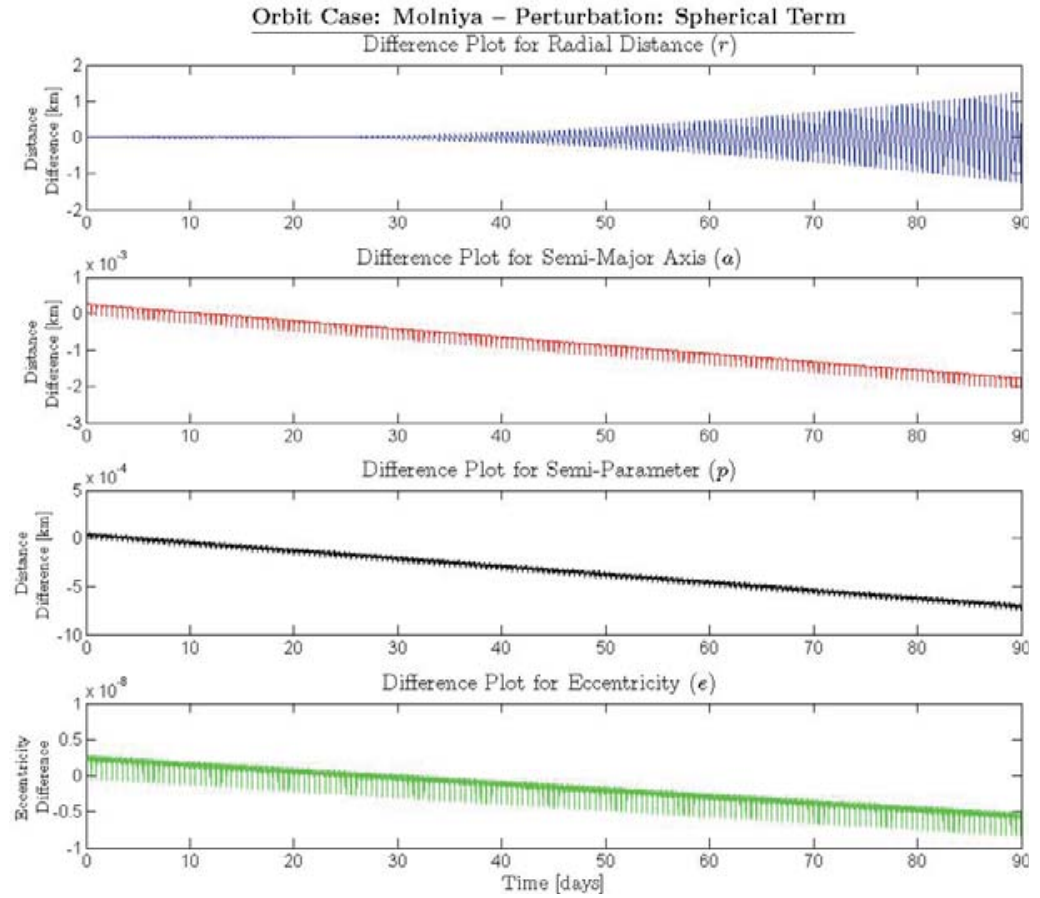

Figure L.2: Molniya Orbit Distance Differences for Spherical Perturbation versus Time 

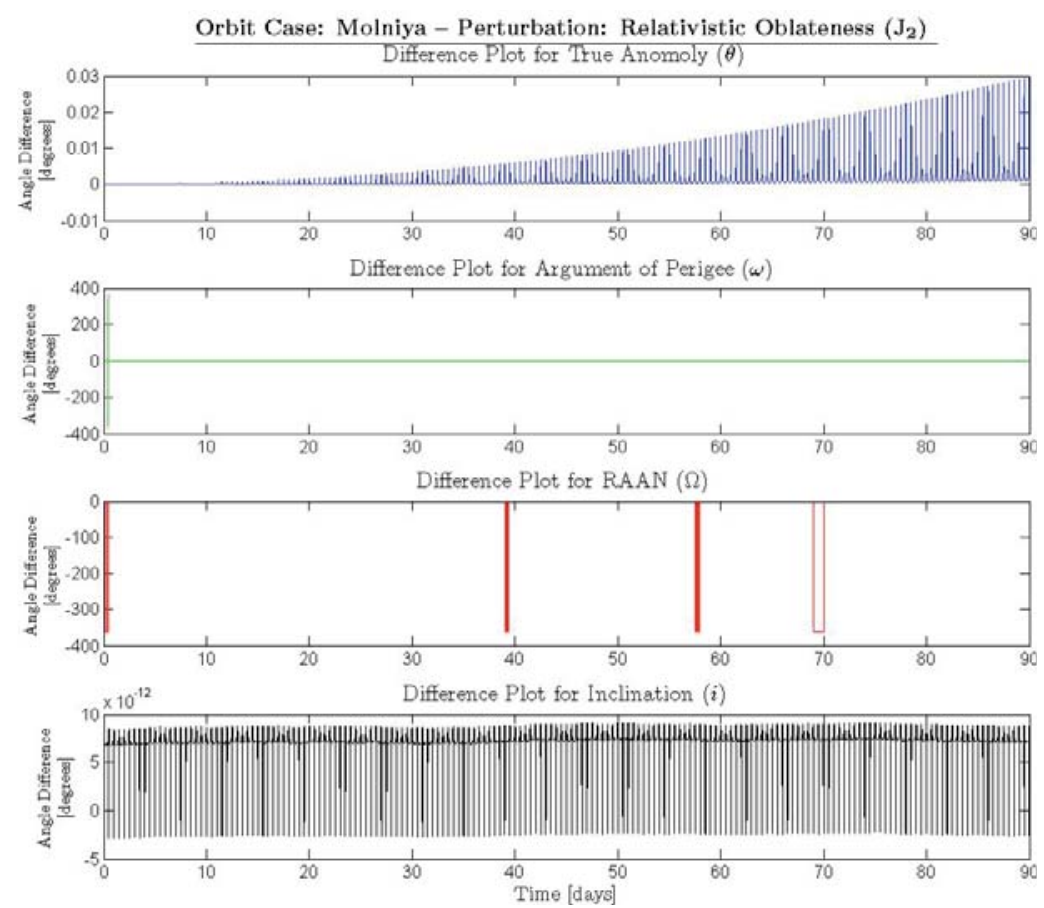

Figure L.3: Molniya Orbit Angular Differences for Relativistic Oblateness versus Time
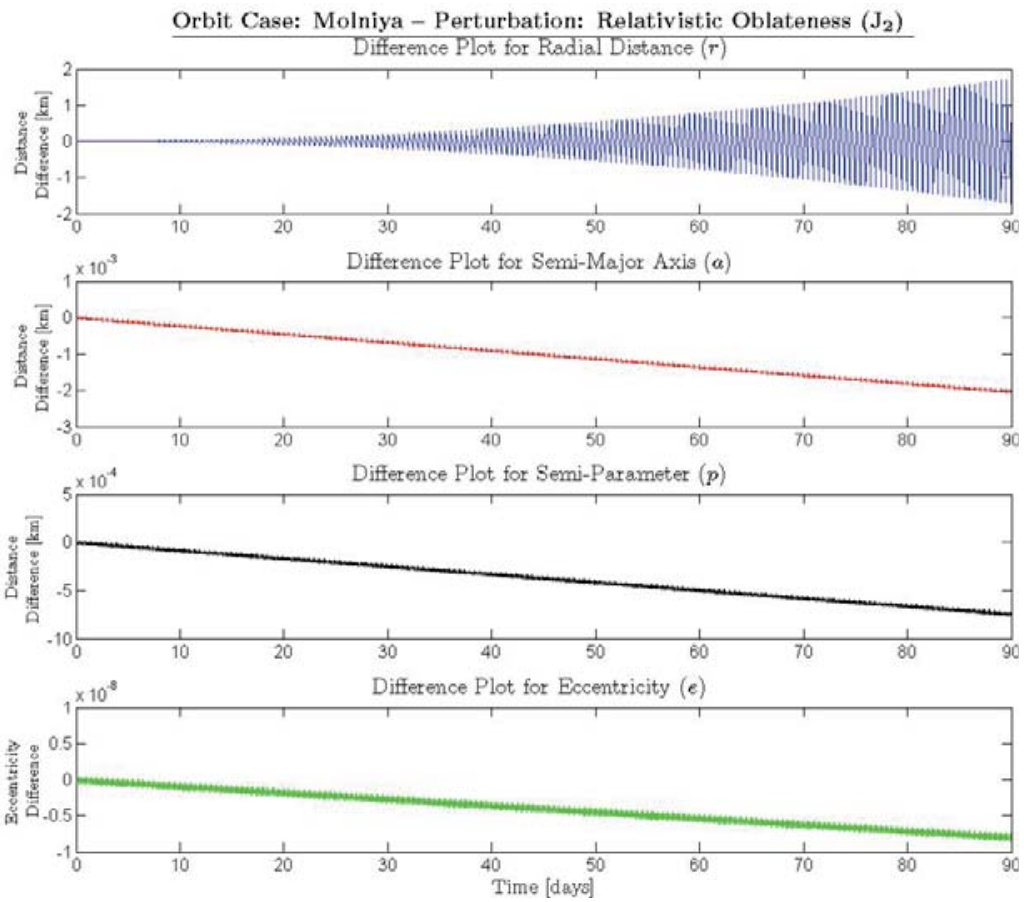

Figure L.4: Molniya Orbit Distance Differences for Relativistic Oblateness versus Time 

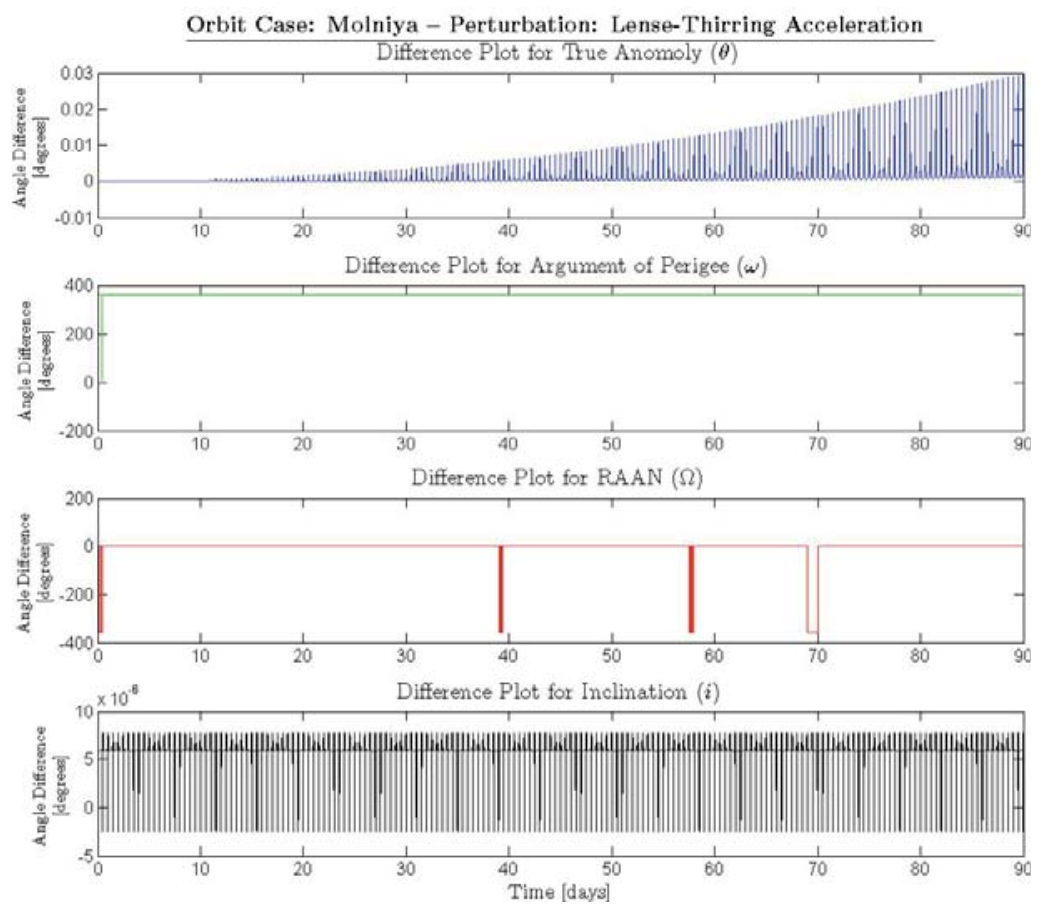

Figure L.5: Molniya Orbit Angular Differences for Lense-Thirring versus Time
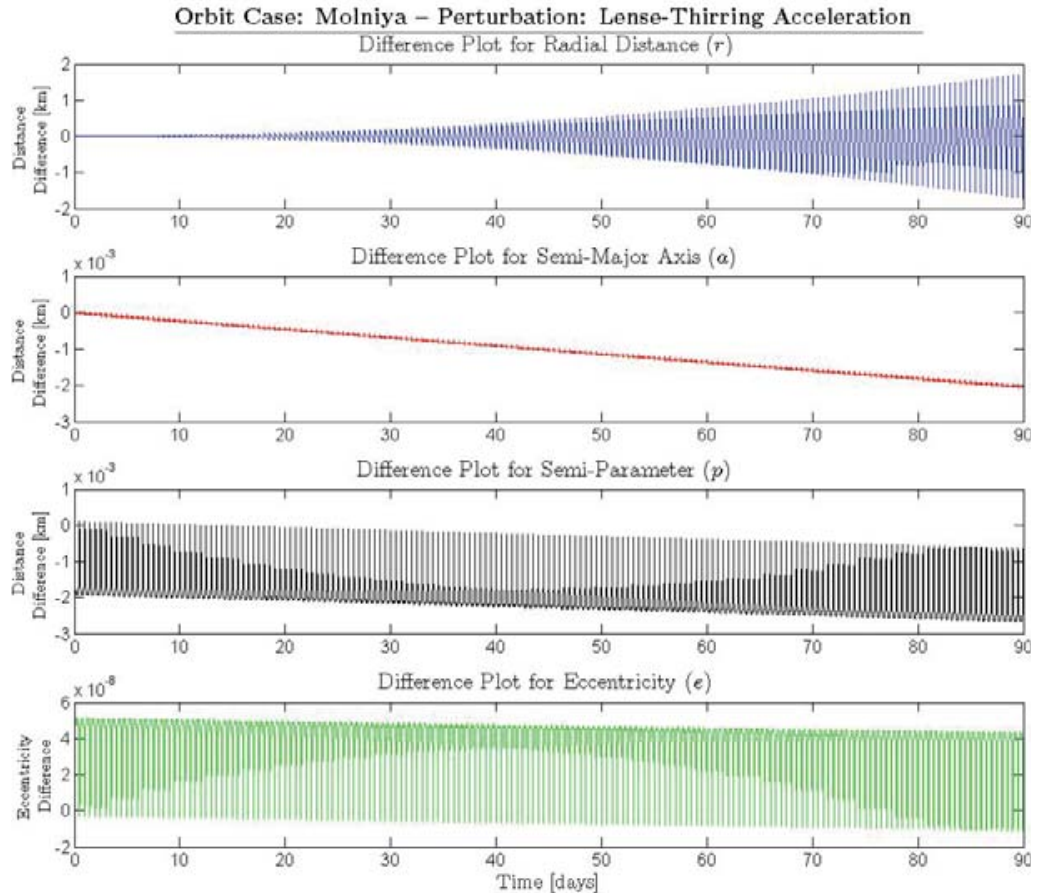

Figure L.6: Molniya Orbit Distance Differences for Lense-Thirring versus Time 

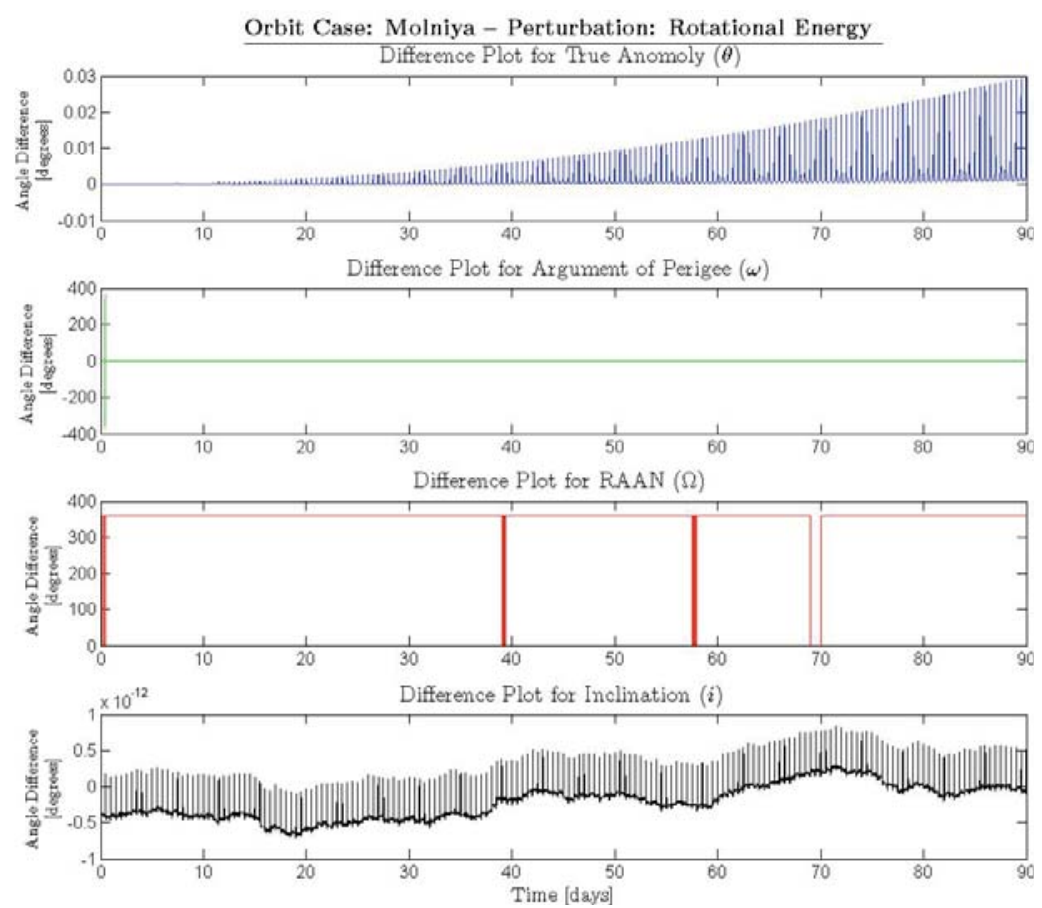

Figure L.7: Molniya Orbit Angular Differences for Rotational Energy versus Time
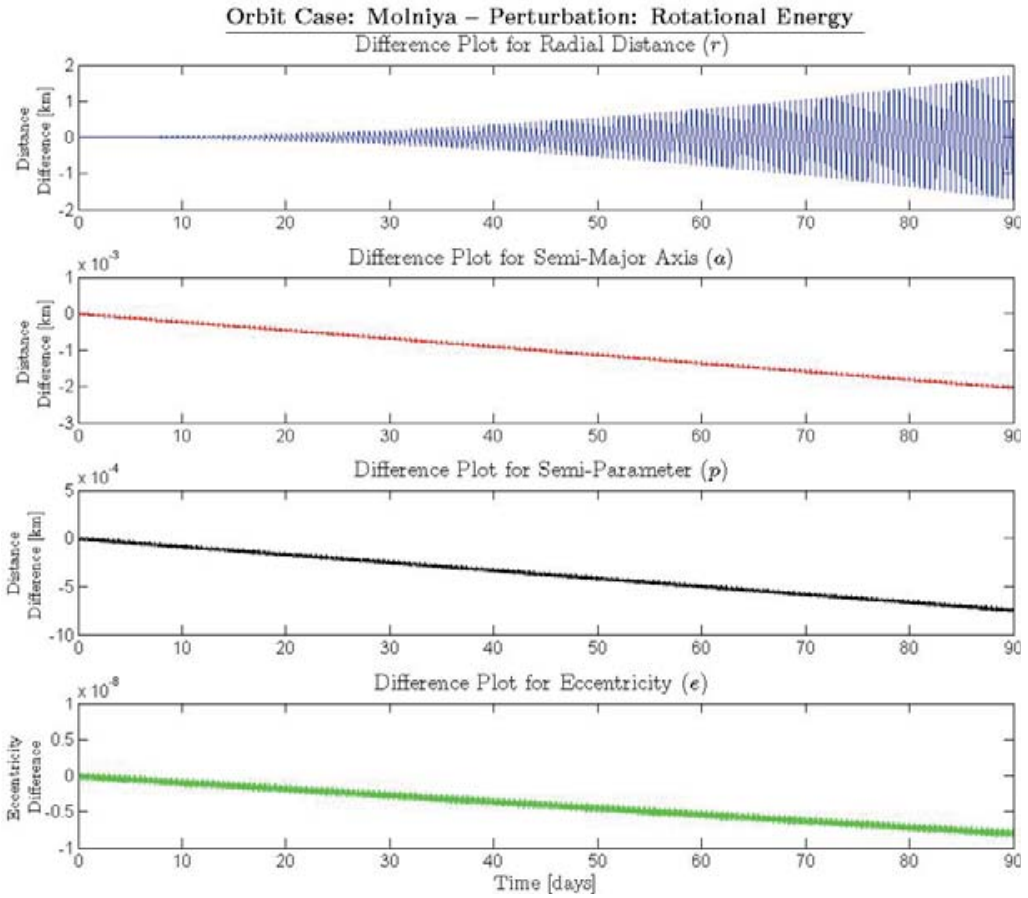

Figure L.8: Molniya Orbit Distance Differences for Rotational Energy versus Time 

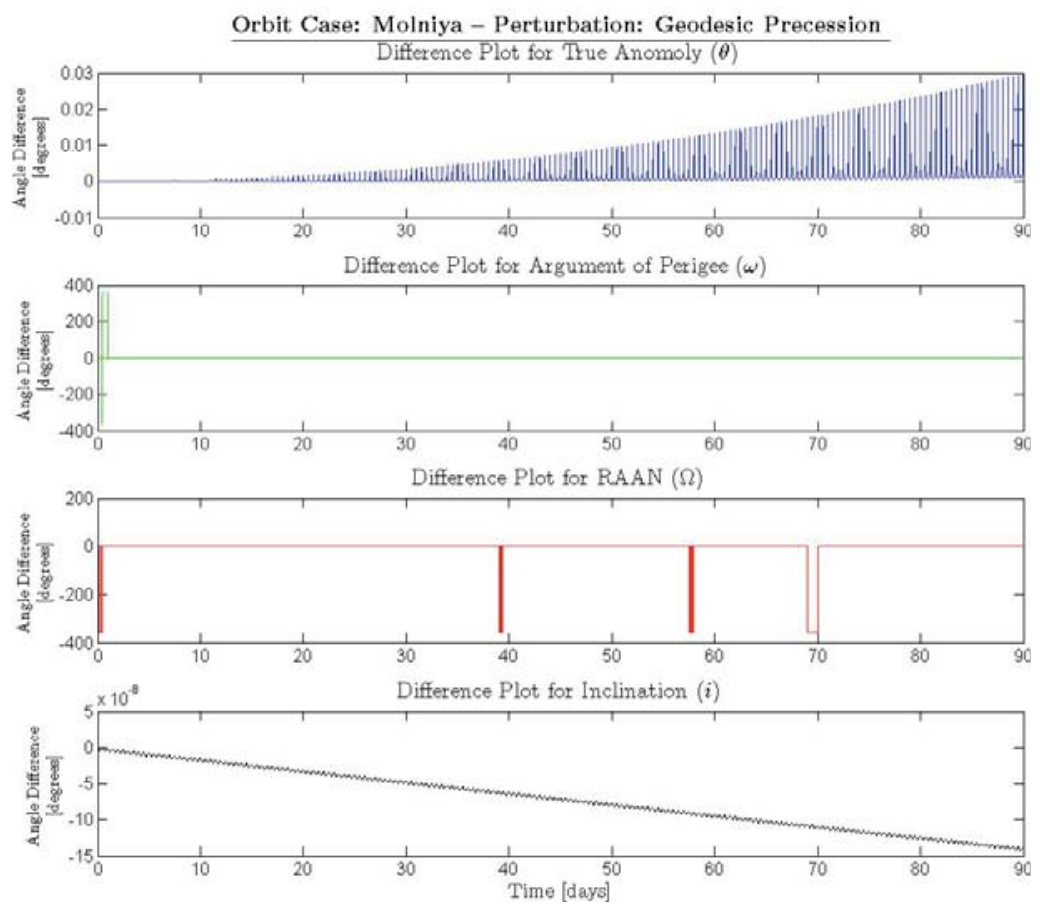

Figure L.9: Molniya Orbit Angular Differences for Geodesic versus Time
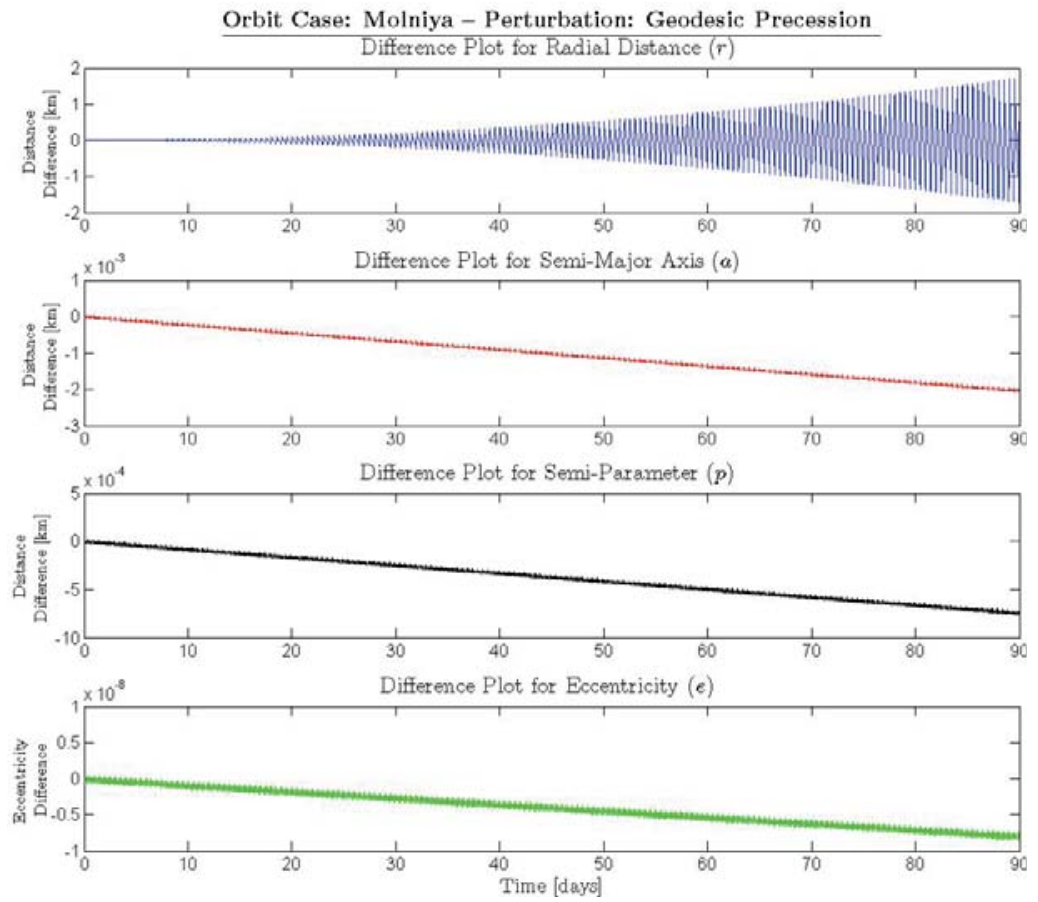

Figure L.10: Molniya Orbit Distance Differences for Geodesic versus Time 


\section{L.2 Specific Force Data}

The graphical data shown in Figures L.11 through L.15 are the specific forces that arise from the relativistic perturbations being applied to the Molniya orbit. The minimum, maximum, mean, and median values of these specific forces has been given in Table L.3 for ease of comparison.

Table L.3: Specific Force Magnitude Data Summary for Molniya Orbit Case

\begin{tabular}{|c|c|c|c|c|c|c|}
\cline { 2 - 7 } \multicolumn{1}{c|}{} & \multicolumn{5}{c|}{ Perturbations } \\
\cline { 2 - 7 } \multicolumn{1}{c|}{} & Spherical & $\begin{array}{c}\text { Lense- } \\
\text { Thirring }\end{array}$ & $\begin{array}{c}\text { Relativistic } \\
\text { Oblateness }\end{array}$ & $\begin{array}{c}\text { Rotational } \\
\text { Energy }\end{array}$ & $\begin{array}{c}\text { Geodesic } \\
\text { Precession }\end{array}$ & Units \\
\hline Min & $3.2469 \mathrm{E}-14$ & $9.3869 \mathrm{E}-13$ & $1.6441 \mathrm{E}-18$ & $1.3032 \mathrm{E}-20$ & $3.5722 \mathrm{E}-15$ & \\
\hline Max & $5.2480 \mathrm{E}-12$ & $2.005 \mathrm{E}-9$ & $1.8775 \mathrm{E}-14$ & $4.1027 \mathrm{E}-16$ & $3.4849 \mathrm{E}-14$ & $\frac{\mathrm{m}}{\mathrm{s}^{2}}$ \\
\hline Mean & $6.1399 \mathrm{E}-13$ & $1.4957 \mathrm{E}-10$ & $8.5347 \mathrm{E}-16$ & $1.7590 \mathrm{E}-17$ & $1.5058 \mathrm{E}-14$ & $1.3299 \mathrm{E}-14$ \\
\hline Median & $9.6261 \mathrm{E}-14$ & $2.8797 \mathrm{E}-12$ & $3.6663 \mathrm{E}-18$ & $4.1475 \mathrm{E}-20$ & \\
\hline
\end{tabular}
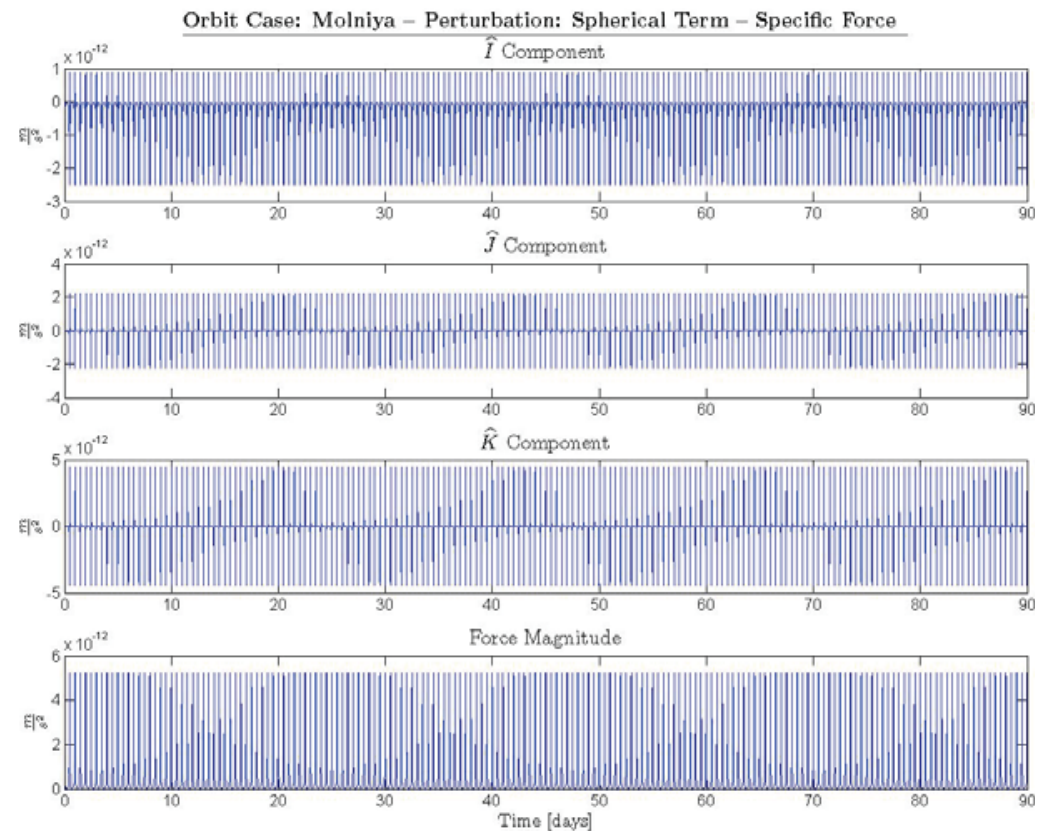

Figure L.11: Molniya Orbit Specific Force for Spherical Perturbation versus Time 

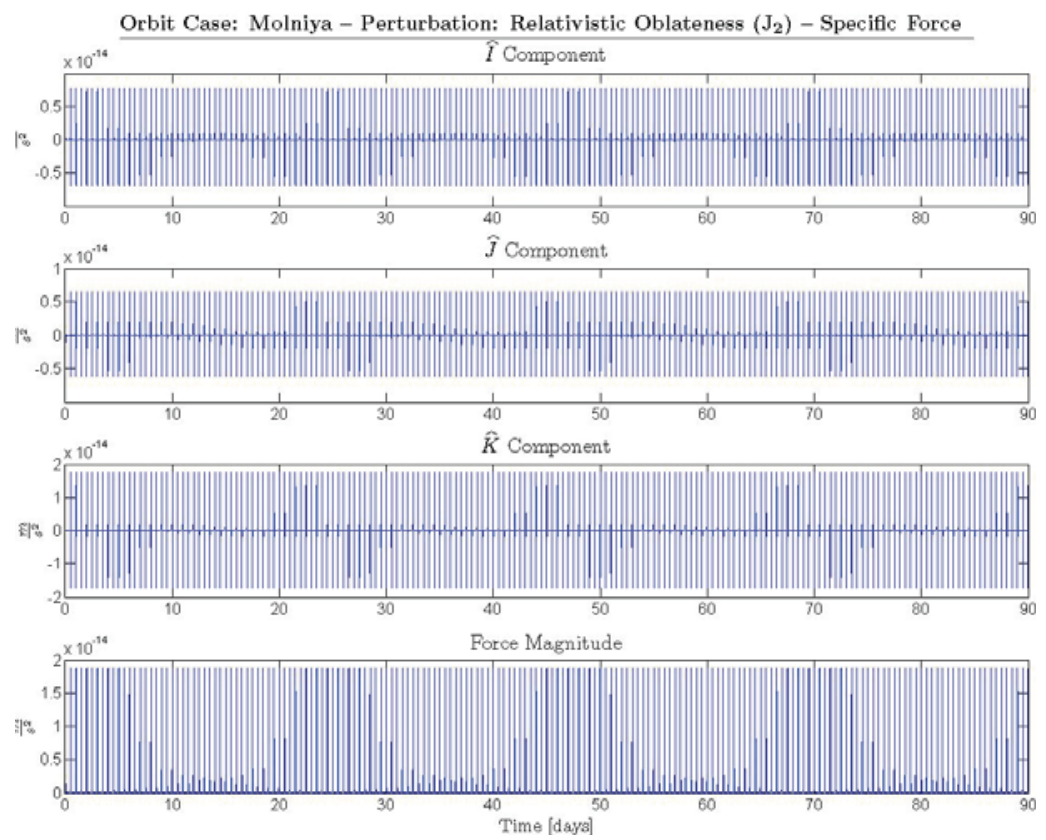

Figure L.12: Molniya Orbit Specific Force for Relativistic Oblateness versus Time
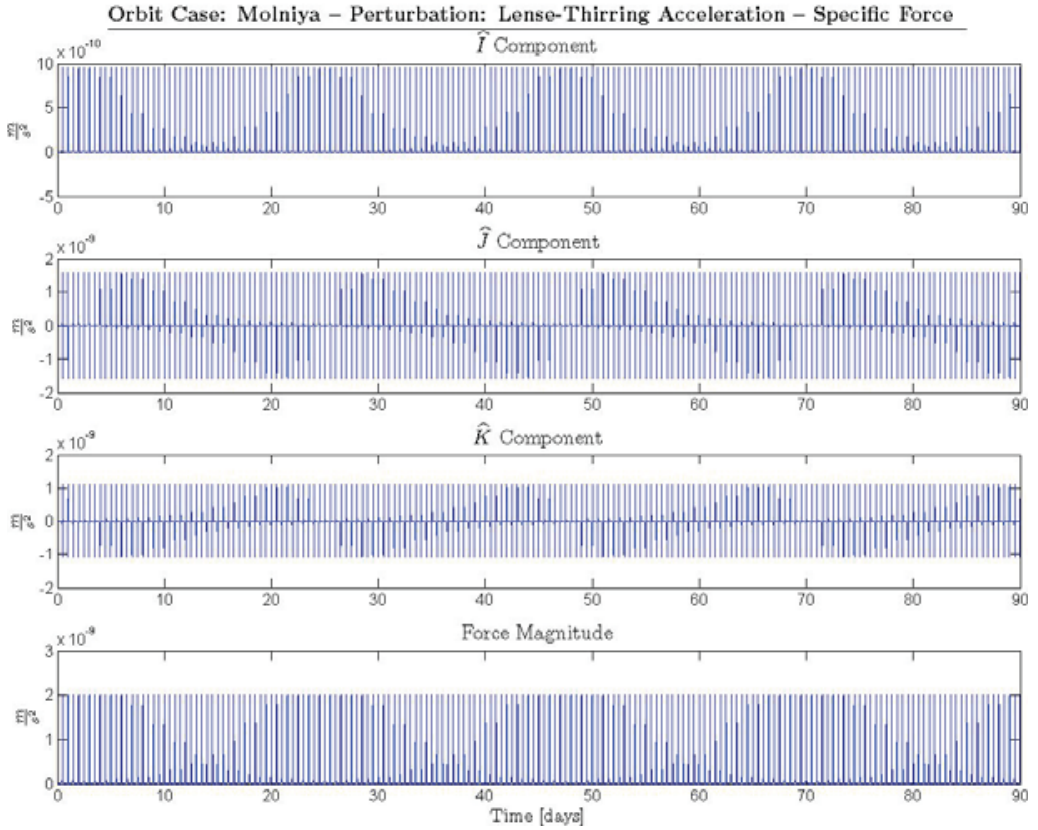

Figure L.13: Molniya Orbit Specific Force for Lense-Thirring versus Time 

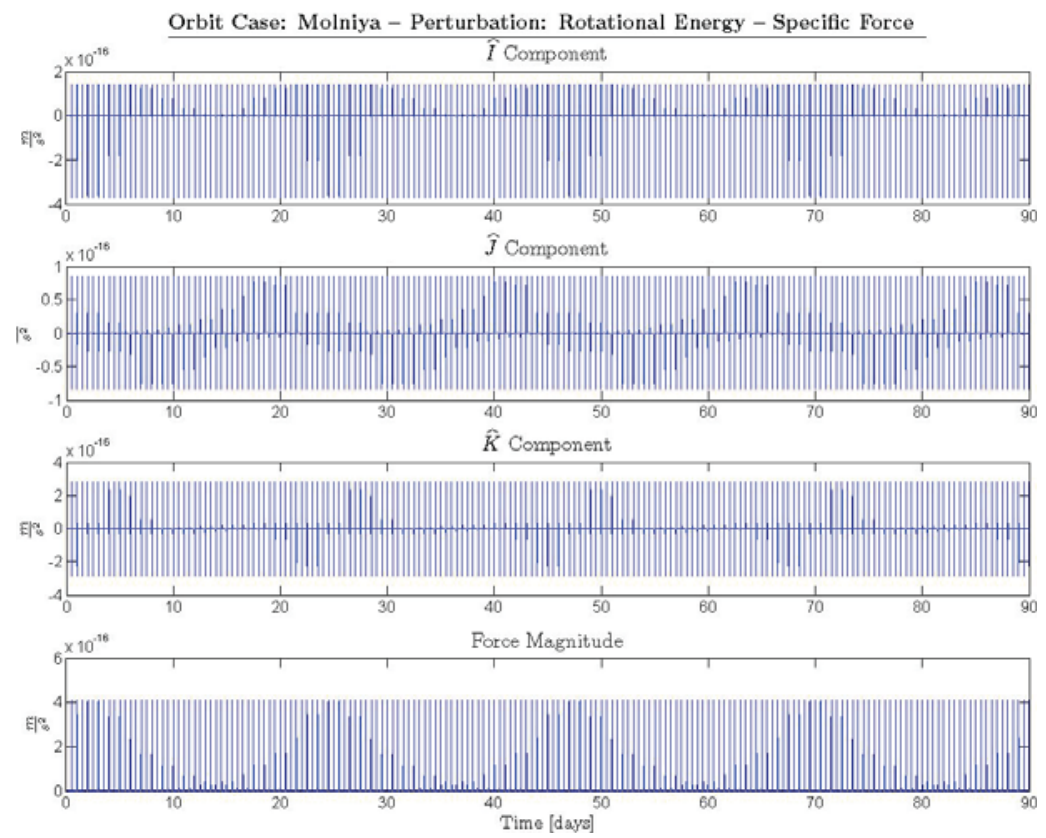

Figure L.14: Molniya Orbit Specific Force for Rotational Energy versus Time
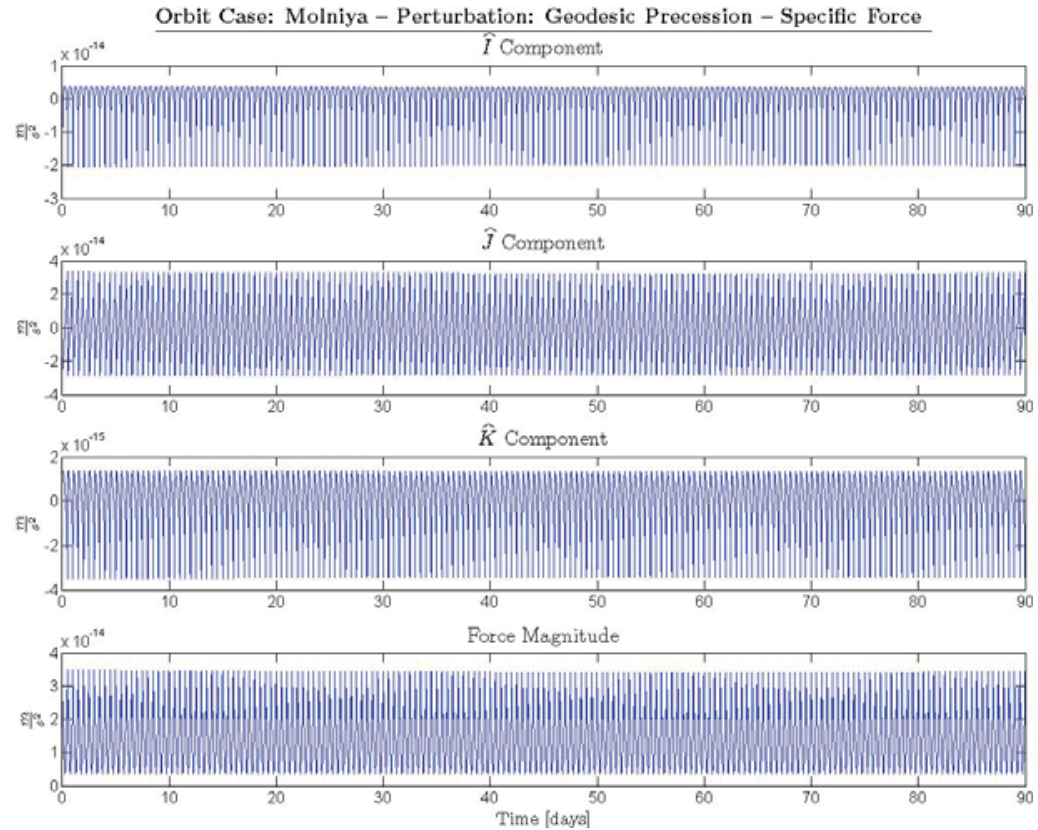

Figure L.15: Molniya Orbit Specific Force for Geodesic versus Time 


\section{Bibliography}

[1] Miguel Alcubierre. The warp drive: hyper-fast travel within general relativity. Classical Quantum Gravity, 11:L73-L77, 1994.

[2] Arthur E. Bryson, Jr. and Yu-Chi Ho. Applied Optimal Control: Optimization, Estimation, and Control. Taylor \& Francis, revised edition, 1975.

[3] Jim Bailey, Mark Foord, Bob Heeter, and Neal Singer. Earth-bound 'star' impersonates blackhole, neutron star: Z, which reaches temperatures of the Sun, to help astronomers interpret Chandra data, November 1999.

[4] Leon Blitzer. Astronautics 453 - Handbook of Orbital Perturbations. University of Arizona.

[5] Bronisław Jakubczyk and Witold Respondek, editors. Geometry of Feedback and Optimal Control. Number 207 in Pure and Appied Mathematics: A Series of Monographs and Textbooks. Marcel Dekker, Inc., New York, New York, first edition, 1998.

[6] Chia-Chun "George" Chao. Applied Orbit Perturbation and Maintenance. The Aerospace Press, El Segundo, California, 2005.

[7] Willem de Sitter. On Einstein's Theory of Gravitation, and its Astronomical Consequences (first paper). Monthly Notices of the Royal Astronomical Society, LXXVI(9):699-728, Supplement 1916.

[8] Willem de Sitter. On Einstein's Theory of Gravitation, and its Astronomical Consequences (second paper). Monthly Notices of the Royal Astronomical Society, LXXVII(2):155-184, December 1916.

[9] Willem de Sitter. On Einstein's Theory of Gravitation, and its Astronomical Consequences (third paper). Monthly Notices of the Royal Astronomical Society, LXXVIII(1):3-28, November 1917.

[10] Ray D'Inverno. Introducing Einstein's Relativity. Oxford University Press, Great Clarendon Street, Oxford OX2 6DP, 1992. 
[11] Jügen Ehlers and Robert Geroch. Equation of motion of small bodies in relativity. Annals of Physics, 309:232-236, 2004.

[12] A. Eistein and L. Infeld. The Evolution of Physics. The Cambridge Library of Modern Science. Cambridge University Press, Bently House, Euston Road; London, N.W. I, 1938.

[13] A. Eistein, L. Infeld, and B. Hoffmann. The Gravitational Equations and the Problem of Motion. The Annals of Mathematics, Second Series, 39(1):65 - 100, January 1938.

[14] Pamela A. Geisler. Planetary Orbits in General Relativity. The Astronomical Journal, 68(9):715-717, November 1963.

[15] Herbert Goldstein. Classical Mechanics. Addison-Wesley Publishing Company, Inc., Reading, Massachusetts, U.S.A., (fifth printing) first edition, December 1957.

[16] Sanford Walton Groesberg. Advanced Mechanics. John Wiley and Sons, Inc., New York, New York, first edition, 1968.

[17] Walter Hohmann. The Attainability of Heavenly Bodies. NASA: Technical Translation, Washington D.C., November 1960.

[18] The Indian Academy of Sciences. The Gravitational Equations of Motion in Relativity, volume 14 A, Bangalore City, March 1942. The Bangalore Press.

[19] Erwin Kreyszig. Differential Geometry. Dover Books on Mathematics. Dover Publications, Inc., New York, New York, dover edition, 1991.

[20] Derek F. Lawden. Optimal Trajectories for Space Navigation. Butterworths \& Co. Ltd., London, UK, first edition, 1963.

[21] Martin W. Lo. The InterPlanetary Superhighway and the Origins Program. 2001.

[22] Martin W. Lo and Shane D. Ross. The Lunar $L_{1}$ Gateway: Portal to the Stars and Beyond. In AIAA Space 2001 Conference. AIAA, 2001.

[23] Charles W. Misner, Kip S. Thorne, and John Archibald Wheeler. Gravitation. W. H. Freeman and Company, San Francisco, 1973.

[24] Paul A. Murad. Hyper-Light Dynamics and the Effects of Relativity, Gravity, Electricity and Magnetism. Acta Astronautica, 47(2-9):575-587, July-November 2000. 
[25] Jorge Nocedal and Stephen J. Write. Numerical Optimization. Springer Series in Operations Research and Financial Engineering. Springer Science + Business Media, LLC, New York, New York, second edition, 2000.

[26] Øyvind Grøn and Sigbjørn Hervik. Einstein's General Theory of Relativity with Modern Applications in Cosmology. Springer Science + Business Media, LLC, 223 Spring Street, New York, NY 10013, USA, 2007.

[27] L. A. Pars. An Introduction to the Calculus of Variations. Heinemann Educational Books Ltd., London, first edition, 1962.

[28] Glenn Earl Peterson. Estimation of the Lense-Thirring Precession Using LaserRanged Satellites. Ph.D. dissertation, The University of Texas at Austin, May 1997.

[29] Michael H. Soffel. Relativity in Astrometry, Celestial Mechanics and Geodesy. Astronomy and Astrophysics Library. Springer-Verlag, Berlin, 1989.

[30] I. S. Sokolnikoff. Tensor Analysis - Theory and Applications. Applied Mathematics Series. John Wiley and Sons, Inc., New York, New York, first edition, July 1958.

[31] Frank D. Stacey and Paul M. Davis. Physics of the Earth. Cambridge University Press, New York, New York, fourth edition, 2008.

[32] David A. Vallado. Fundamentals of Astrodynamics and Applications. Space Technology Libary. Microcosm Press and Springer, third edition, 2007.

[33] Gerry Yonas and Neal Singer. High-Output Sandia Accelerator Able to Predict Nuclear Blast Physics, December 1996. 